\title{
Trinion conformal blocks from topological strings
}

\author{
Ioana Coman, ${ }^{a, b}$ Elli Pomoni ${ }^{a}$ and Joerg Teschner ${ }^{a, c}$ \\ ${ }^{a}$ DESY, Theory Group, \\ Notkestraße 85, Building 2a, 22607 Hamburg, Germany \\ ${ }^{b}$ Institute of Physics, University of Amsterdam, \\ 1098 XH Amsterdam, the Netherlands \\ ${ }^{c}$ Department of Mathematics, University of Hamburg, \\ Bundesstrasse 55, 20146 Hamburg, Germany \\ E-mail: i.comanlohi@uva.nl, elli.pomoni@desy.de, \\ joerg.teschner@desy.de
}

ABSTRACT: In this paper we investigate the relation between conformal blocks of Liouville CFT and the topological string partition functions of the rank one trinion theory $T_{2}$. The partition functions exhibit jumps when passing from one chamber in the parameter space to another. Such jumps can be attributed to a change of the integration contour in the free field representation of Liouville conformal blocks. We compare the partition functions of the $T_{2}$ theories representing trifundamental half hypermultiplets in $N=2, d=4$ field theories to the partition functions associated to bifundamental hypermultiplets. We find that both are related to the same Liouville conformal blocks up to inessential factors. In order to establish this picture we combine and compare results obtained using topological vertex techniques, matrix models and topological recursion. We furthermore check that the partition functions obtained by gluing two $T_{2}$ vertices can be represented in terms of a four point Liouville conformal block. Our results indicate that the $T_{2}$ vertex offers a useful starting point for developing an analog of the instanton calculus for SUSY gauge theories with trifundamental hypermultiplets.

Keywords: Conformal and W Symmetry, Conformal Field Theory, Supersymmetry and Duality, Topological Strings

ArXiv ePrint: 1906.06351 


\section{Contents}

1 Introduction 1

2 The topological vertex computation $\quad 4$

2.1 The computation 4

$\begin{array}{lll}2.2 & \text { Resummation into a product formula } & 7\end{array}$

2.3 Dependence on the choice of preferred direction 9

$\begin{array}{lll}2.4 & T_{2} \text { web with non-empty external Young tableaux } & 10\end{array}$

3 The 4D limit $\quad 11$

$\begin{array}{ll}3.1 \text { A useful factorisation } & 11\end{array}$

$\begin{array}{ll}3.2 & \text { The limit of the regular part } \\ 3.32\end{array}$

$\begin{array}{lll}3.3 & \text { Renormalising the singular part } & 13\end{array}$

$\begin{array}{ll}3.4 & \text { Extending the domain of definition of the } T_{2} \text { partition functions }\end{array}$

4 Integral representation of the $T_{2}$ partition function $\quad 16$

4.1 Imposing the specialisation condition to the topological strings $\quad 17$

$\begin{array}{ll}\text { 4.2 The matrix integral as a sum of residues } & 18\end{array}$

$\begin{array}{ll}\text { 4.3 The free field representation for } q \text {-Liouville } & 19\end{array}$

5 The $q \rightarrow 1$ limit of the Matrix integral $\quad 20$

5.1 A simple example 21

$\begin{array}{ll}5.2 & \text { The matrix integral } \\ 5.3 & 22\end{array}$

5.3 The matrix integral as a Selberg integral 23

6 Comparison with the strip vertex $\quad 24$

$\begin{array}{lll}6.1 & \text { Topological vertex computation } & 25\end{array}$

$\begin{array}{ll}6.2 \text { Comparison } & 26\end{array}$

7 B-model picture $\quad \mathbf{2 7}$

$\begin{array}{lll}7.1 & \text { Relation between mirror curves of strip and } T_{2} & 27\end{array}$

$\begin{array}{lll}7.2 & \text { Geometric transitions and matrix models } & 28\end{array}$

$\begin{array}{lll}7.3 & \text { Topological recursion } & 30\end{array}$

$\begin{array}{lll}8 & T_{N} \text { versus conformal blocks } & 31\end{array}$

9 Gluing $T_{2}$ building blocks vs. strips $\quad 34$

9.1 $\mathrm{SU}(2)$ four-flavour $\mathcal{N}=1$ theory in 5D from gluing of strips 36

9.2 $\mathrm{SU}(2)$ four-flavour $\mathcal{N}=1$ theory in $5 \mathrm{D}$ from gluing of $T_{2}$ blocks 37

9.3 Mapping to Virasoro four point conformal blocks 40 
10.1 Using the $T_{2}$-vertex in the AGT-correspondence 41

$\begin{array}{ll}10.2 \text { Higher rank } & 42\end{array}$

A Special functions $\quad 44$

$\begin{array}{ll}\text { A.1 The limit } q \rightarrow 1 & 47\end{array}$

B Variants of Jackson integrals and the $q \rightarrow 1$ limit $\quad 48$

$\begin{array}{lll}\text { C Integral representation of the } T_{N} \text { partition function } & 49\end{array}$

$\begin{array}{ll}\text { D Summary of important formulae } & 51\end{array}$

\section{Introduction}

The discovery of relations between $\mathcal{N}=2, d=4$ supersymmetric gauge theories and conformal field theory by Alday, Gaiotto and Tachikawa [1] has stimulated a large amount of work. Such relations have meanwhile been proven for the linear or circular quiver gauge theories built from vector multiplets and hypermultiplets in the (bi-)fundamental and adjoint representation of the gauge group $[2,3]$.

In a parallel development, the $\mathcal{N}=2, d=4$ supersymmetric field theories of class $\mathcal{S}$ have been introduced in $[4,5]$. The theories of class $\mathcal{S}$ are classified by punctured Riemann surfaces $C$ and Lie-algebras $\mathfrak{g}$ of ADE-type. In the cases where $\mathfrak{g}=A_{1}$ there exists a Lagrangian representation for any pants decomposition of $C$. It involves vector multiplets for any cutting curve, and certain matter multiplets associated to any pair of pants. By considering the S-dualities of theories in class $\mathcal{S}$ associated to changes of pants decomposition [4] one can identify the half-hypermultiplet in the tri-fundamental representation of $[\mathrm{SU}(2)]^{3}$ as the most natural candidate for the field theory associated to the pairs of pants, see [6] for a review. For the cases $\mathfrak{g}=A_{N}$ with $N>1$ one generically expects to find non-Lagrangian theories as the building blocks associated to pairs of pants.

Almost all of the available checks or proofs [2, 7-14] of AGT-type relations have been performed in the linear quiver theories obtained by using fundamental or bi-fundamental hypermultiplets of $\mathrm{U}(N)$ instead of tri-fundamental half-hypermultiplets of $\mathrm{SU}(N)$. For these cases one can use the instanton calculus for $\mathrm{U}(N)$-theories developed in $[15,16]$. The instanton calculus for half-hypermultiplets in the tri-fundamental representation of $\mathrm{SU}(2)$ is much less developed. Apart from the work [17] we are not aware of any direct checks of AGT-type relations in class $\mathcal{S}$ theories built from tri-fundamental half-hypermultiplets. The difficulties become even more severe in many cases with $\mathfrak{g}=A_{N-1}, N>2$. The theories associated to the pair of pants will then generically be non-Lagrangian, preventing us from using any of the conventional tools based on Lagrangian QFT. 
A possible way out is offered by the geometric engineering of supersymmetric field theories within string theory. Many such field theories can be described as certain scaling limits of string theory on $R^{4} \times M$, with $M$ being a toric CY manifold [18, 19]. In such cases one expects that the instanton partition functions coincide with the topological string partition functions computable with the help of the (refined) topological vertex [2022]. Combined with the AGT-correspondence one gets relations between topological string partition functions and conformal blocks for which more direct explanations have been proposed in $[23,24] .^{1}$ Dualities are expected to relate string theory on $R^{4} \times M$ for various toric CY $M$ to intersecting 5-brane systems called brane webs. The toric diagrams encoding the geometry of the CY manifold $M$ coincide with the web diagrams describing the intersection patterns of the brane webs [27, 28], simplifying the identification of the gauge theories describing the relevant scaling limits. This line of reasoning has lead to a family of candidates for string-theoretic descriptions of the class $\mathcal{S}$ theories associated to the three-punctures spheres, which are often referred to as the $T_{N}$-theories and associated to certain web diagrams proposed in [29]. One might hope that the refined topological vertex offers tools for the computation of partition functions in cases where the gaugetheoretic instanton calculi are not available or hard to use. This line of thought has been the motivation for a series of investigations [30-33].

A considerable amount of evidence is available supporting the role of the $T_{N}$ vertex as a building block of class $\mathcal{S}$ theories. ${ }^{2}$ Analogs of the AGT-correspondence have been found relating instanton partition functions in five-dimensional supersymmetric gauge theories to q-deformations of conformal blocks for the $W_{N}$-algbra. Using free field representations for the q-deformed conformal blocks one can derive such relations in many cases [38, 39].

One should note, however, that these results are not immediately applicable to the study of the AGT-correspondence for the class $\mathcal{S}$ theories. ${ }^{3}$ It seems to us, in particular, that the relation between the $T_{N}$ theories and conformal blocks is not well-understood. There are basic open questions even in the simplest case $N=2$. The partition function of the $T_{2}$ theory has been calculated in [40] where it was observed that the absolute value of this partition function is closely related to the three point function of Liouville CFT. This gives a first piece of evidence for a relation between the $T_{2}$ theory and conformal blocks on three-punctured sphere, but it is pretty far from proving such a relation. At the moment it does not even seem to be known if the gluing of $T_{2}$ vertices reproduces the conformal blocks on spheres with more than three punctures.

It is interesting to compare the $T_{2}$ vertex with another composite vertex which has been discussed in the literature, often referred to as the strip [41]. In the context of

\footnotetext{
${ }^{1}$ For related important works see [25], as well as the review [26] and references therein.

${ }^{2}$ The web diagrams of [29] reproduce the correct the dimensions of Coulomb and Higgs branches, the $c$ and $a$ anomaly coefficients [34], the correct Seiberg Witten curves [29, 30] and when identified with the toric diagrams of CY manifolds, they reproduce the correct 5D superconformal index [35] (the partition function on $\left.\mathbb{S}^{4} \times \mathbb{S}^{1}\right)$ of the $5 \mathrm{D} T_{N}$ trinion theories [30, 31], including the $E_{6,7,8}$ symmetry enhancement [30, 31, 36]. See [37] for a review.

${ }^{3}$ The five-dimensional analogs of the AGT correspondence studied in the literature either concern a deformation of the linear quiver theories, or dual versions of this correspondence in the sense of fiber-base duality. It is not known how to go beyond the well-understood case of linear quiver theories in this way.
} 
geometric engineering one may naturally associate the strip diagrams with fundamentalor bi-fundamental hypermultiplets. It is well-known that the strip partition functions represent the basic building blocks for the instanton partition functions in linear or circular quiver gauge theories. For this case one has a clear understanding of the relation between the instanton partition functions and conformal blocks.

This state of affairs naturally raises two basic questions:

(a) Can we demonstrate AGT-type relations for the class $\mathcal{S}$-theories engineered from the $T_{2}$ vertex?

(b) Does the AGT-correspondence distinguish between class $\mathcal{S}$-theories built from different types of vertices?

These are the two main questions we will aim to address in this paper. We thereby hope to alleviate slightly the tension between the basic role of the $T_{2}$-vertex in class $\mathcal{S}$ theories on the one hand, and the shortage of hard evidence for the AGT relation involving the $T_{2}$-vertex on the other hand. We also view our investigation as a natural warmup for investigations of the $T_{N}$-theories with $N \geq 2$. On the way we will address the following questions.

1. The limits giving the partition functions of $4 \mathrm{D}$ gauge theories from the topological string partition functions are tricky. It turns out that the partition functions diverge in this limit, and need to be renormalised ${ }^{4}$ to get meaningful results. As usual, there can be freedom in the definition of the renormalisation prescription. This raises the question how predictive this approach can be. This becomes particularly important in the cases $N>2$, but even for $N=2$ we did not find a discussion of this issue in the literature.

2. Can we obtain the known instanton partition functions of non-abelian gauge theories by gluing $T_{2}$ partition functions? Having such a check would confirm that the $T_{2}$ vertex can indeed play the expected role as a building block for more class $\mathcal{S}$ theories.

3. What is the precise map between the Kähler moduli of the toric CY $M$ defining the $T_{2}$ vertex and the parameters labelling bases for the three point conformal blocks?

On the way we take the opportunity to close some gaps in the existing literature. The paper is organised as follows. We first review the derivation of the $T_{2}$ topological strings partition function, discussing its resummation into a product formula in section 2. This is followed in section 3 by an analysis of the $4 \mathrm{D}$ limit and a prescription for taking this limit in a meaningful way. Section 4 then presents an alternative route towards deriving this limit, rewriting the partition function as a matrix integral. Here we also compare the integral formulation to the three point conformal block of $q$-Liouville CFT. We then proceed in section 5 to take the $4 \mathrm{D}$ limit and rewrite the matrix integral as a Selberg integral. As a building block, the $T_{2}$ needs to be compared to a better studied counterpart, commonly

\footnotetext{
${ }^{4}$ Here we use the word renormalisation in a wider sense, as a procedure to define the limit of a formally divergent quantity.
} 

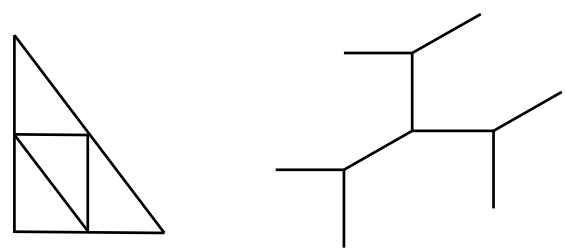

Figure 1. The toric diagram (on the left) and its dual 5-brane web diagram (on the right) for the $T_{2}$ geometry, the supersymmetric $\mathbb{C}^{2} / \mathbb{Z}_{2} \times \mathbb{Z}_{2}$ orbifold.

referred to as the strip. This analysis can be found in section 6 , followed by section 7 which comments on this picture from the B-model point of view. Section 8 then offers some words on the generalisation of this story to the higher rank case for the $T_{N}$ theories. Afterwards, section 9 follows this discussion with explicitly gluing $T_{2}$ blocks and comparing to the gluing of strips. The appendices then gather the definitions and properties of ubiquitous special functions. For the reader's (and our) convenience we have collected some of the key formulae in appendix D.

\section{The topological vertex computation}

In this section we review the computation of the topological sting partition function in [30].

The theory of $[\mathrm{SU}(2)]^{3}$ half-hypermultiplet in the tri-fundamental representation can be obtained from string theory in various ways. There exists a five dimensional uplift of this theory compactified on $\mathbb{S}^{1}$, which can be constructed in type IIB string theory via the $(p, q)$ web 5-brane diagram [42, 43] depicted in figure 1, due to [29]. Equivalently, this theory can be constructed in M-theory/type IIA string theory on Calabi-Yau threefolds via geometric engineering $[18,19]$. The toric diagram of the corresponding $\mathbb{C} \times \mathbb{C}^{2} / \mathbb{Z}_{2} \times \mathbb{Z}_{2}$ Calabi-Yau manifold has the same shape as the dual of the $T_{2}$ web diagram [27]. The partition function of the $T_{2}$ theory and any $\mathcal{N}=2$ theory geometrically engineered with a toric Calabi-Yau compactification can be obtained by computing the topological string partition function on the Calabi-Yau [16, 44, 45]. A very efficient method to compute 5D Nekrasov partition functions is the refined topological vertex formalism $[21,22,46]$.

\subsection{The computation}

The $T_{2}$ geometry is parametrised by three independent Kähler parameters $Q_{1}, Q_{2}$ and $Q_{3}$ that are related to the $5 \mathrm{D}$ gauge theory parameters $P_{1}, P_{2}, P_{3}$ in figure 2 as

$$
Q_{1} Q_{3}=\frac{P_{1}^{(1)}}{P_{1}^{(2)}}=P_{1}^{2}, \quad Q_{1} Q_{2}=\frac{P_{2}^{(1)}}{P_{2}^{(2)}}=P_{2}^{2}, \quad Q_{2} Q_{3}=\frac{P_{3}^{(1)}}{P_{3}^{(2)}}=P_{3}^{2}
$$

which are then related to the $4 \mathrm{D}$ gauge theory parameters as $-R^{-1} \ln P_{i}$, with $R$ the radius of the circle of the 5th dimension. The topological partition function is also a function of the parameters $q, t$ which are the $5 \mathrm{D}$ version of Nekrasov's Omega background

$$
q=e^{-R \epsilon_{1}}, \quad t=e^{R \epsilon_{2}} .
$$




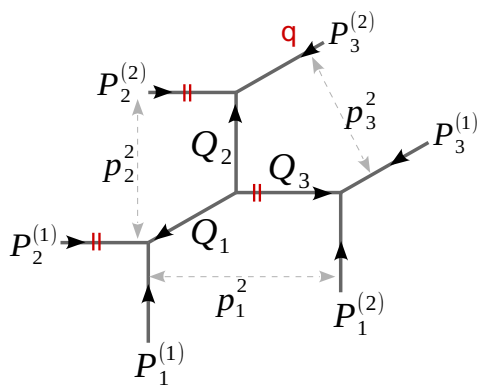

Figure 2. The 5-brane web diagram for $T_{2}$ with the choice of the preferred direction and the Kähler parameters. The edges along the preferred direction are marked by two red strips.

For completeness we sketch the rules for reading off the refined topological string partition function from a dual toric diagram. The procedure is very similar to calculating Feynman diagrams where we associate functions of the Kähler parameters, the Omega background as well as Young diagrams to the edges and vertices of the dual toric diagram. The topological partition function is then obtained as the product of all the edge and vertex factors, summed over all possible partitions associated with the internal edges.

In order to compute the refined topological string partition function, using the refined topological vertex formalism we first need to pick a preferred direction on the toric diagram. This is denoted with two red lines in figure 2. The final result for the closed topological string partition function is conjectured to not depend on the choice of the preferred direction $[21,47]$. However, the closed topological string partition function corresponds to the full correlation function. We are interested in its holomorphic half, the conformal block obtained from the open topological string amplitude. The later carries the information of the choice of the preferred direction $[48,49]$.

Returning to the rules for computing the refined topological string partition function, to each edge we associate a partition $\mu$. The partitions associated to external lines of the diagram are empty. The edge factor is the function that we associate to each edge:

$$
\text { edge factor } \stackrel{\text { def }}{=}(-Q)^{|\mu|} \times \text { framing factor. }
$$

For the $T_{2}$ toric diagram depicted in figure 2 the only framing factors which we need to use are trivial, equal to one. In general, the framing factor is given by

$$
f_{\mu}(t, q)=(-1)^{n_{e}|\mu|} t^{\frac{n_{e}\left\|\mu^{t}\right\| \|^{2}}{2}} q^{-\frac{n_{e}\|\mu\|^{2}}{2}}, \tilde{f}_{\mu}(t, q)=(-1)^{n_{e}|\mu|} t^{\frac{n_{e}\left(\left.\left\|\mu^{t}\right\|\right|^{2}+|\mu|\right)}{2}} q^{-\frac{n_{e}\left(\|\mu\| \|^{2}+|\mu|\right)}{2}}
$$

with $n_{e}=\operatorname{det}\left(\vec{v}_{\text {in }} \vec{v}_{\text {out }}\right)$ an integer defined like in figure 3 and framing factors assigned to edges like in figure 4.

The refined topological vertex is the function that we associate to each vertex:

$$
C_{\mu \nu \lambda}(t, q)=q^{\frac{\|\nu\|^{2}+\|\lambda\|^{2}}{2}} t^{-\frac{\left\|\nu^{t}\right\|^{2}}{2}} \tilde{Z}_{\lambda}(t, q) \sum_{Y}\left(\frac{q}{t}\right)^{\frac{|Y|+|\mu|-|\nu|}{2}} s_{\mu^{t} / Y}\left(t^{-\rho} q^{-\lambda}\right) s_{\nu / Y}\left(q^{-\rho} t^{-\lambda^{t}}\right)
$$



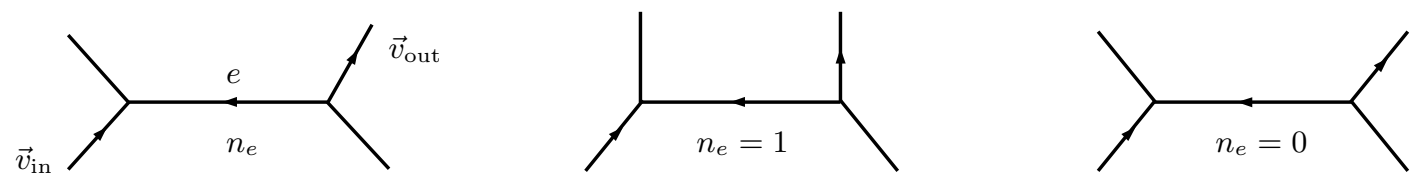

Figure 3. The integer $n_{e}$ associated to the edge $e$ in the graph is defined as $n_{e}=\operatorname{det}\left(\vec{v}_{\text {in }} \vec{v}_{\text {out }}\right)$. Thus for the graphs in the middle and on the right, this yields $n_{e}=1$ and $n_{e}=0$, respectively.
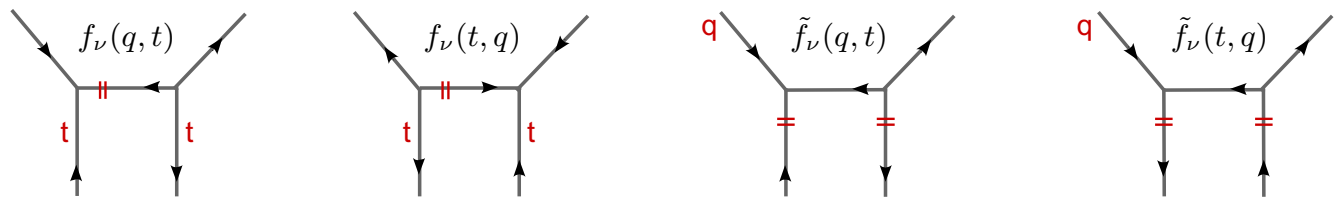

Figure 4. The assignment of framing factors to edges of a web diagram.

where we have used the functions $\tilde{Z}_{\lambda}(t, q)$ defined in equation (A.20) in appendix A. The $s_{\mu / \nu}(x)$ are skew-Schur functions of the possibly infinite vector $x=\left(x_{1}, \ldots\right)$. We use the notation that for a partition $\nu=\left(\nu_{1}, \nu_{2}, \ldots\right)$, the vector $t^{-\rho} q^{-\nu}$ is given by

$$
t^{-\rho} q^{-\nu}=\left(t^{\frac{1}{2}} q^{-\nu_{1}}, t^{\frac{3}{2}} q^{-\nu_{2}}, t^{\frac{5}{2}} q^{-\nu_{3}}, \ldots\right) .
$$

The topological string partition function is a sum over the partitions $\left\{Y_{1}, \cdots, Y_{M}\right\}$ of the $M$ internal edges of the toric diagram

$$
\mathcal{Z}^{\text {top }}=\sum_{Y_{1}, \cdots, Y_{M}} \prod_{\text {edges }} \text { edge factor } \times \prod_{\text {vertices }} \text { vertex factor. }
$$

For the $T_{2}$ web depicted in figure 1 the refined topological string partition function is

$$
\mathcal{Z}_{T_{2}}^{\text {top }}=\mathcal{Z}_{2}^{\text {top }}(\boldsymbol{Q})=\sum_{\boldsymbol{R}} \prod_{i=1}^{3}\left(-Q_{i}\right)^{\left|R_{i}\right|} C_{R_{1}^{t} \emptyset \emptyset}(q, t) C_{\emptyset R_{2}^{t} \emptyset}(q, t) C_{\emptyset \emptyset R_{3}^{t}}(q, t) C_{R_{1} R_{2} R_{3}}(t, q),
$$

where $\boldsymbol{Q}=\left(Q_{1}, Q_{2}, Q_{3}\right)$ and $R_{i}$, for $i=1,2,3$, is the Young diagram decorating the edge with corresponding Kähler parameter $Q_{i}$. The $T_{2}$ geometry is special and following the work of [50] for the unrefined partition function we can perform some of the sums and achieve a more compact way to write (2.8). For that we use the following identities involving Schur functions

$$
\begin{aligned}
\sum_{Y} Q^{|Y|} s_{Y / R_{1}}( & \left.{ }^{-\rho} q^{-\nu_{1}}\right) s_{Y^{t} / R_{2}}\left(q^{-\rho} t^{-\nu_{2}}\right)= \\
& =\mathcal{R}_{\nu_{2} \nu_{1}}(-Q ; t, q) \sum_{Y} Q^{\left|R_{1}\right|+\left|R_{2}\right|-|Y|} s_{R_{2}^{t} / Y}\left(t^{-\rho} q^{-\nu_{1}}\right) s_{R_{1}^{t} / Y^{t}}\left(q^{-\rho} t^{-\nu_{2}}\right),
\end{aligned}
$$

and

$$
\begin{aligned}
\sum_{Y} Q^{|Y|} s_{Y / R_{1}}\left(t^{-\rho} q^{-\nu_{1}}\right) s_{Y / R_{2}}\left(q^{-\rho} t^{-\nu_{2}}\right)= \\
=\mathcal{R}_{\nu_{2} \nu_{1}}(Q ; t, q)^{-1} \sum_{Y} Q^{\left|R_{1}\right|+\left|R_{2}\right|-|Y|} s_{R_{2} / Y}\left(t^{-\rho} q^{-\nu_{1}}\right) s_{R_{1} / Y}\left(q^{-\rho} t^{-\nu_{2}}\right),
\end{aligned}
$$


where the new function $\mathcal{R}_{\nu_{2} \nu_{1}}(Q ; t, q)$ is defined in (A.21). The final result is [30]

$\mathcal{Z}_{2}^{\text {top }}(\boldsymbol{Q})=\mathcal{M}\left(Q_{1} Q_{2}\right) \sum_{R_{3}}\left(-Q_{3}\right)^{\left|R_{3}\right|} t^{\frac{\left\|R_{3}^{t}\right\|^{2}}{2}} q^{\frac{\left\|R_{3}\right\|^{2}}{2}} \tilde{Z}_{R_{3}^{t}}(q, t) \tilde{Z}_{R_{3}}(t, q) \mathcal{R}_{R_{3}^{t} \emptyset}\left(Q_{2} ; t, q\right) \mathcal{R}_{\emptyset R_{3}}\left(Q_{1} ; t, q\right)$

and using (A.9) and (A.19)-(A.22) can be brought to the form

$$
\begin{aligned}
& \mathcal{Z}_{2}^{\text {top }}\left(P_{1}, P_{2}, P_{3} ; t, q\right)= \\
& =\frac{\mathcal{M}\left(P_{2}^{2}\right)}{\mathcal{M}\left(\sqrt{\frac{t}{q}} \frac{P_{2} P_{3}}{P_{1}}\right) \mathcal{M}\left(\sqrt{\frac{t}{q}} \frac{P_{1} P_{2}}{P_{3}}\right)} \sum_{\nu}\left(\sqrt{\frac{t}{q}} \frac{P_{1} P_{3}}{P_{2}}\right) \frac{|\nu| \mathcal{N}_{\nu \emptyset}\left(\sqrt{\frac{t}{q}} \frac{P_{2} P_{3}}{P_{1}}\right) \mathcal{N}_{\emptyset \nu}\left(\sqrt{\frac{t}{q}} \frac{P_{1} P_{2}}{P_{3}}\right)}{\mathcal{N}_{\nu \nu}(t / q)} .
\end{aligned}
$$

Note. Here we have used the relations of the three Kähler parameters $Q_{1}, Q_{2}$ and $Q_{3}$ to the 5 D parameters $P_{1}, P_{2}, P_{3}(2.1)$ and the shorthand notations $\mathcal{M}(Q)=\mathcal{M}(Q ; t, q)$ and $\mathcal{N}_{R P}(Q)=\mathcal{N}_{R P}(Q ; t, q)$.

\subsection{Resummation into a product formula}

In this section we will show how the partition function of the $T_{2}$ theory can be brought to an infinite product formula [40]

$$
\mathcal{Z}_{2}^{\text {top }}(\boldsymbol{Q})=\prod_{i, j=1}^{\infty} \frac{\left(1-Q_{1} Q_{2} Q_{3} q^{i-\frac{1}{2}} t^{j-\frac{1}{2}}\right) \prod_{k=1}^{3}\left(1-Q_{k} q^{i-\frac{1}{2}} t^{j-\frac{1}{2}}\right)}{\left(1-Q_{1} Q_{2} q^{i} t^{j-1}\right)\left(1-Q_{1} Q_{3} q^{i-1} t^{j}\right)\left(1-Q_{2} Q_{3} q^{i} t^{j-1}\right)}
$$

We begin with the formula (2.12) that was derived using the topological vertex formalism. It is useful to write it, using (A.11), as $\mathcal{Z}_{2}^{\text {top }}(\boldsymbol{Q})=\mathcal{Z}_{2}^{\text {prod }}(\boldsymbol{Q}) \check{\mathcal{Z}}_{2}(\boldsymbol{Q})$, with

$$
\check{\mathcal{Z}}_{2}(\boldsymbol{Q})=\sum_{R}\left(-Q_{3}\right)^{|R|} q^{\frac{\|R\|^{2}}{2}} t^{\frac{\left\|R^{t}\right\|^{2}}{2}} \prod_{(i, j) \in R} \frac{\left(1-Q_{1} \sqrt{\frac{t}{q}} q^{1-j} t^{i-1}\right)\left(1-Q_{2} \sqrt{\frac{q}{t}} q^{j-1} t^{1-i}\right)}{\left(1-q^{R_{i}-j+1} t^{R_{j}^{t}-i}\right)\left(1-q^{R_{i}-j} t^{R_{j}^{t}-i+1}\right)},
$$

and we denote by $\mathcal{Z}_{2}^{\text {prod }}=\mathcal{M}\left(P_{2}^{2}\right) / \mathcal{M}\left(P_{2} P_{3} / P_{1} v\right) \mathcal{M}\left(P_{1} P_{2} / P_{3} v\right)$ the part of the partition function that is already in product form. This sum is a formal series (for which we have not given a proof of convergence yet) and it has the form

$$
\check{\mathcal{Z}}_{2}(\boldsymbol{Q})=\sum_{k=0}^{\infty}\left(-Q_{3}\right)^{k} \mathrm{P}_{k}\left(Q_{1}, Q_{2}\right)
$$

where $\mathrm{P}_{k}\left(Q_{1}, Q_{2}\right)$ is a degree $k$ polynomial in $Q_{1}$ and $Q_{2}$ which can also be explicitly written as

$$
\mathrm{P}_{k}\left(Q_{1}, Q_{2}\right)=\sum_{\ell=0}^{k} Q_{1}^{\ell} \mathrm{P}_{k \ell}\left(Q_{2}\right)=\sum_{\ell, m=0}^{k} Q_{1}^{\ell} Q_{2}^{m} \mathrm{P}_{k \ell}
$$

The idea of the proof is to find a function that has the same formal series expansion, but which is known to be convergent. 
Note that the polynomials $\mathrm{P}_{k}\left(Q_{1}, Q_{2}\right)$ have degree $k$ in both variables $Q_{1}$ and $Q_{2}$. In order to determine the polynomials $\mathrm{P}_{k}$ uniquely, it suffices to know their values for $(k+1)^{2}$ different values of $Q_{1}$ and $Q_{2}$. We will consider the values of $Q_{1}$ and $Q_{2}$ defined by

$$
Q_{1}(N)=q^{N} \sqrt{\frac{q}{t}}, \quad Q_{2}(M)=q^{M} \sqrt{\frac{t}{q}}, \quad \text { for } N, M \in \mathbb{Z} .
$$

For these cases we can further rewrite expression (2.15) using a specialisation of the Macdonald symmetric polynomials

$$
P_{R}\left(t^{N-\frac{1}{2}}, t^{N-\frac{3}{2}}, \cdots, t^{\frac{1}{2}} ; q, t\right)=t^{\frac{\left\|R^{t}\right\|^{2}}{2}} \prod_{(i, j) \in R} \frac{\left(1-q^{j-1} t^{N+1-i}\right)}{\left(1-q^{R_{i}-j} t^{R_{j}^{t}-i+1}\right)}
$$

from [51], equation (6.11') on page 337. Then, by further using the Cauchy formula

$$
\sum_{R} Q^{|R|} P_{R}(x ; q, t) P_{R}(y ; t, q)=\prod_{i, j=1}^{\infty}\left(1+Q x_{i} y_{j}\right)
$$

for the Macdonald symmetric polynomials, we can rewrite

$$
\begin{aligned}
& \check{\mathcal{Z}}_{2}\left(Q_{1}(N), Q_{2}(M), Q_{3}\right)= \\
& \quad=\sum_{R}\left(-Q_{3}\right)^{|R|} P_{R^{t}}\left(q^{N-\frac{1}{2}}, q^{N-\frac{3}{2}}, \cdots, q^{\frac{1}{2}} ; t, q\right) P_{R}\left(t^{M-\frac{1}{2}}, t^{M-\frac{3}{2}}, \cdots, t^{\frac{1}{2}} ; q, t\right) \\
& \quad=\prod_{i=1}^{N} \prod_{j=1}^{M}\left(1-Q_{3} q^{i-\frac{1}{2}} t^{j-\frac{1}{2}}\right) .
\end{aligned}
$$

It is not hard to find a function $\mathcal{Y}\left(Q_{1}, Q_{2}, Q_{3}\right)$ such that the values $\mathcal{Y}\left(Q_{1}(N), Q_{2}(M), Q_{3}\right)$ are given by the right side of (2.20),

$$
\mathcal{Y}\left(Q_{1}, Q_{2}, Q_{3}\right):=\prod_{i, j=1}^{\infty} \frac{\left(1-Q_{3} q^{i-\frac{1}{2}} t^{j-\frac{1}{2}}\right)\left(1-Q_{1} Q_{2} Q_{3} q^{i-\frac{1}{2}} t^{j-\frac{1}{2}}\right)}{\left(1-Q_{1} Q_{3} q^{i-1} t^{j}\right)\left(1-Q_{2} Q_{3} q^{i} t^{j-1}\right)} .
$$

The function $\mathcal{Y}\left(Q_{1}, Q_{2}, Q_{3}\right)$ is meromorphic in all three variables. In order to see that the function $\mathcal{Y}\left(Q_{1}, Q_{2}, Q_{3}\right)$ is analytic in a neighbourhood of $Q_{3}=0$ one may represent it in the form

$$
\mathcal{Y}(\boldsymbol{Q})=\frac{\mathcal{M}\left(Q_{1} Q_{3} \frac{t}{q}\right) \mathcal{M}\left(Q_{2} Q_{3}\right)}{\mathcal{M}\left(Q_{3} \sqrt{\frac{t}{q}}\right) \mathcal{M}\left(Q_{1} Q_{2} Q_{3} \sqrt{\frac{t}{q}}\right)},
$$

with the function $\mathcal{M}(Q) \equiv \mathcal{M}(Q ; t, q)$ having a representation as an exponential function of a convergent power series,

$$
\mathcal{M}(Q ; t, q)=\exp \left[\sum_{m=1}^{\infty} \frac{Q^{m}}{m} \frac{q^{m}}{\left(1-t^{m}\right)\left(1-q^{m}\right)}\right],
$$

which converges for all $t$ and all $q$ provided that $|U|<q^{-1+\theta(|q|-1)} t^{\theta(|t|-1)}$, where $\theta(x)$ denotes the step function which is $\theta(x)=1$ if $x>0$ and $\theta(x)=0$ if $x \leq 0$. 

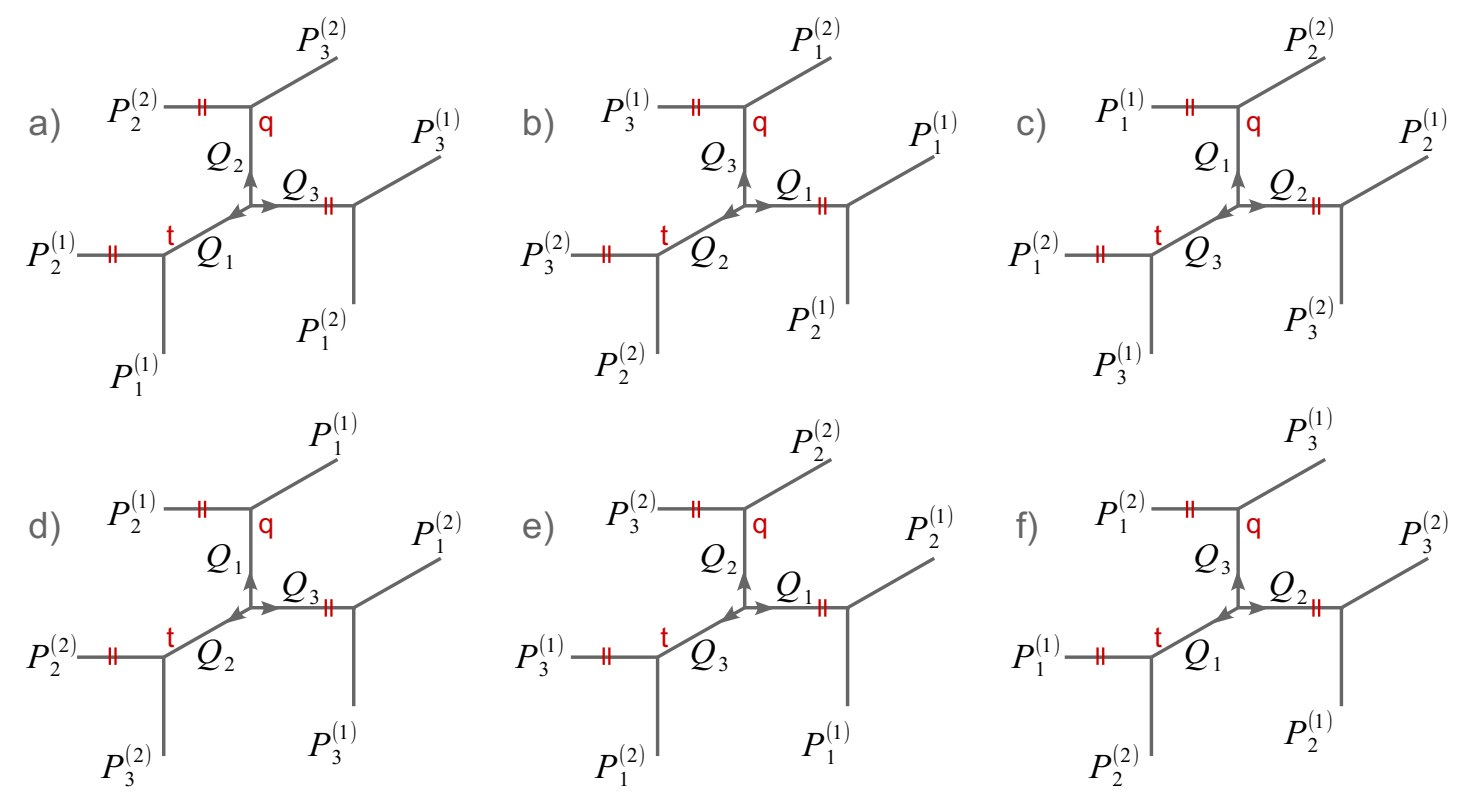

Figure 5. The upper figures $a), b$ ) and $c$ ) correspond to the three different ways to choose the preferred direction while the three lower figures $d$ ), $e$ ) and $f$ ) correspond to the three reflections over the middle axis labeled by the preferred direction.

The expansion of $\mathcal{Y}\left(Q_{1}, Q_{2}, Q_{3}\right)$ in powers of $Q_{3}$ has the same form as the expansion $(2.15), \mathcal{Y}\left(Q_{1}, Q_{2}, Q_{3}\right)=\sum_{k=0}^{\infty} Q_{3}^{k} \mathrm{P}_{k}^{\prime}\left(Q_{1}, Q_{2}\right)$. The equations $\check{\mathcal{Z}}_{2}\left(Q_{1}(N), Q_{2}(M), Q_{3}\right)=$ $\mathcal{Y}\left(Q_{1}(N), Q_{2}(M), Q_{3}\right)$ imply that the polynomials $\mathrm{P}_{k}\left(Q_{1}, Q_{2}\right)$ and $\mathrm{P}_{k}^{\prime}\left(Q_{1}, Q_{2}\right)$ agree on an infinite set of values. We must therefore have $\mathrm{P}_{k}\left(Q_{1}, Q_{2}\right)=\mathrm{P}_{k}^{\prime}\left(Q_{1}, Q_{2}\right)$ for all $k \in \mathbb{Z}, k \geq 0$. But this implies that $\check{\mathcal{Z}}_{2}\left(Q_{1}, Q_{2}, Q_{3}\right)=\mathcal{Y}\left(Q_{1}, Q_{2}, Q_{3}\right)$, which gives the result we wanted to prove.

\subsection{Dependence on the choice of preferred direction}

To round off the picture let us comment on the dependence on the choice of preferred direction on the web diagram. There are three possible ways of choosing the preferred direction and they correspond to rotating around the middle point of the $T_{2}$ web diagram the lines labeled by the preferred direction, while keeping the conventions of the topological vertex (figure 6) fixed as well as keeping the Kähler parameters fixed to their position. Equivalently, we can keep the preferred direction and the placing of $t, q$ fixed and rotate the positions of the Kähler parameters. This is depicted in figure $5 a$ ), b) and $c$ ). We may note that

$$
\begin{aligned}
& \mathcal{Z}_{2, b}^{\text {top }}\left(Q_{1}, Q_{2}, Q_{3}\right)=\mathcal{Z}_{2}^{\text {top }}\left(Q_{2}, Q_{3}, Q_{1}\right) \\
& \mathcal{Z}_{2, c}^{\text {top }}\left(Q_{1}, Q_{2}, Q_{3}\right)=\mathcal{Z}_{2}^{\text {top }}\left(Q_{3}, Q_{1}, Q_{2}\right)
\end{aligned} \quad \mathcal{Z}_{2, a}^{\text {top }}\left(Q_{1}, Q_{2}, Q_{3}\right) \equiv \mathcal{Z}_{2}^{\text {top }}\left(Q_{1}, Q_{2}, Q_{3}\right)
$$



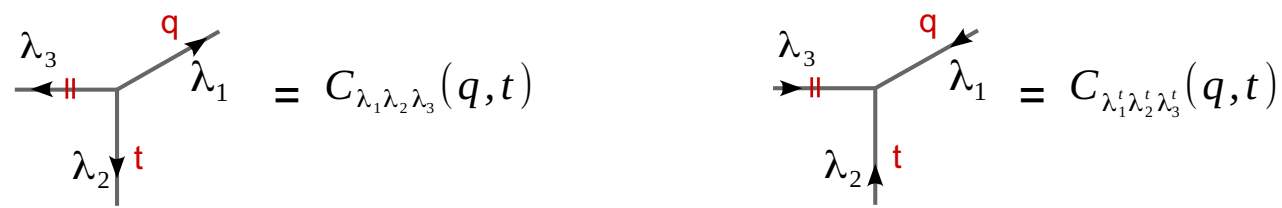

Figure 6. The direction of the arrows determines the way partitions enter the vertex factor. Partitions are counted clockwise to the preferred direction and the variables $t, q$ enter the vertex factor in this order if $t$ is associated with the end of the first edge and $q$ with the end of the second.

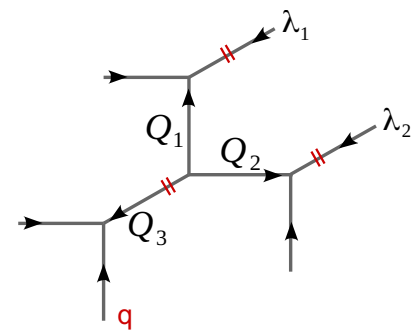

Figure 7. The $T_{2}$ web with non-empty Young diagrams associated to the external $(1,1)$ branes.

Moreover, changing the conventions and defining the vertex factor counterclockwise (instead of clockwise) leads a further possibility. It can be shown that

$$
\mathcal{Z}_{2, d}^{\text {top }}\left(Q_{1}, Q_{2}, Q_{3}\right)=\mathcal{Z}_{2}^{\text {top }}\left(Q_{2}, Q_{1}, Q_{3}\right)
$$

The relation between $\mathcal{Z}_{2, d}^{\text {top }}$ and $\mathcal{Z}_{2}^{\text {top }}$ may be equivalently described by exchanging $t \leftrightarrow q$. In total we get the six different options depicted in figure 6 .

However, inspection of the explicit formula (2.13) reveals that for all of these cases one finds the same functions of $Q_{1}, Q_{2}, Q_{3}$ in the numerator. The denominator factorises into three functions depending separately on only one of the variables $P_{1}, P_{2}$ and $P_{3}$, in the following called leg factors. As will be explained in section 3.4, we will mostly be interested in the part of the result which does not factorise in this way. It will therefore not represent an essential loss of generality for us to consider only $\mathcal{Z}_{2, a}^{\text {top }}\left(Q_{1}, Q_{2}, Q_{3}\right)=\mathcal{Z}_{2}^{\text {top }}\left(Q_{1}, Q_{2}, Q_{3}\right)$ from now on.

\section{$2.4 \quad T_{2}$ web with non-empty external Young tableaux}

In this paper one of our goals is to explicitly check that by gluing two $T_{2}$ vertices we obtain the known instanton partition function of the $5 \mathrm{D} \mathrm{SU}(2)$ gauge theory with four flavours. For that we need to allow the Young diagrams associated to two of the external legs to be non-empty, like in figure 7 . Then the topological strings partition function for the $T_{2}$ diagram is given by

$$
\mathcal{Z}_{2, \vec{\lambda}}^{\text {top }}\left(Q_{1}, Q_{2}, Q_{3} ; t, q\right)=\sum_{\nu} \prod_{i=1}^{3}\left(-Q_{i}\right)^{\left|\nu_{i}\right|} C_{\emptyset \emptyset \nu_{3}^{t}}(q, t) C_{\emptyset \nu_{2}^{t} \lambda_{2}^{t}}(q, t) C_{\nu_{1}^{t} \emptyset \lambda_{1}^{t}}(q, t) C_{\nu_{1} \nu_{2} \nu_{3}}(t, q) .
$$


By applying the formulas (2.9)-(2.10) repeatedly, this expression can be simplified to

$$
\begin{aligned}
\mathcal{Z}_{2, \vec{\lambda}}^{\mathrm{top}}\left(Q_{1}, Q_{2}, Q_{3} ; t, q\right)= & t^{\frac{\left\|\lambda_{1}^{t}\right\|^{2}+\left\|\lambda_{2}^{t}\right\|^{2}}{2}} \tilde{Z}_{\lambda_{1}^{t}}(q, t) \tilde{Z}_{\lambda_{2}^{t}}(q, t) \frac{\mathcal{M}\left(Q_{1} Q_{2}\right)}{\mathcal{M}\left(Q_{1} \sqrt{\frac{t}{q}}\right) \mathcal{M}\left(Q_{2} \sqrt{\frac{t}{q}}\right)} \\
& \sum_{\nu}\left(Q_{3} \sqrt{\frac{q}{t}}\right)^{|\nu|} \frac{\mathcal{N}_{\lambda_{1} \nu}\left(Q_{1} \sqrt{\frac{t}{q}}\right) \mathcal{N}_{\nu \lambda_{2}}\left(Q_{2} \sqrt{\frac{t}{q}}\right)}{\mathcal{N}_{\nu \nu}(1) \mathcal{N}_{\lambda_{1} \lambda_{2}}\left(Q_{1} Q_{2}\right)} .
\end{aligned}
$$

At this point it is currently not known how to perform the summation over $\nu$.

\section{The $4 \mathrm{D}$ limit}

The previous section has reviewed the derivation of the topological strings partition function for the $T_{2}$ brane web. Recall that this partition function is for the $5 \mathrm{D}$ uplift on $\mathbb{S}^{1}$ of the theory describing the $2 \times 2$ free hypermultiplet. We are now going to analyse its $4 \mathrm{D}$ limit, with $\mathbb{S}^{1}$ radius $R \rightarrow 0$. In order to define this limit precisely we find it useful to parametrise the variables $q, t, P_{1}, P_{2}$ and $P_{3}$, as

$$
q=e^{-\epsilon_{1} R}, \quad t=q^{\beta^{2}}, \quad P_{1}^{2}=\frac{t^{\nu}}{t}, \quad P_{2}^{2}=\frac{t^{\mu}}{q}, \quad P_{3}^{2}=\frac{t^{\lambda}}{q} .
$$

The limit of our interest is $R \rightarrow 0$, keeping $\epsilon_{1}, \beta, \lambda, \mu, \nu$ finite. Introducing the notation

$$
s=\frac{1}{2}\left(\lambda+\mu-\nu-\beta^{-2}\right)
$$

allows us to rewrite the expression for $\mathcal{Z}_{2}^{\text {top }} \equiv \mathcal{Z}_{2}^{\text {top }}\left(\lambda, \mu, \nu ; \epsilon_{1}, \beta, R\right)$ in the following useful form

$$
\mathcal{Z}_{2}^{\text {top }}=\frac{1}{\mathcal{M}\left(t^{1+s} / q\right)} \frac{\mathcal{M}\left(t^{\lambda} / q\right) \mathcal{M}\left(t^{\mu} / q\right) \mathcal{M}\left(t^{\nu} / q\right)}{\mathcal{M}\left(t^{\lambda-s} / q\right) \mathcal{M}\left(t^{\mu-s} / q\right) \mathcal{M}\left(t^{\nu+s} / q\right)}
$$

It turns out that each of the functions $\mathcal{M}$ becomes singular in the limit defined above, see appendix A.1 for a discussion. It follows that the function $\mathcal{Z}_{2}^{\text {top }}\left(\lambda, \mu, \nu, \epsilon_{1}, \epsilon_{2}, R\right)$ does not have a finite limit when $R \rightarrow 0$. In the rest of this section we will discuss how a meaningful limit can be defined nevertheless.

\subsection{A useful factorisation}

In the following it will be demonstrated that $\mathcal{Z}_{2}^{\text {top }}$ can be factorised into a singular and a regular factor

$$
\mathcal{Z}_{2}^{\text {top }}\left(\lambda, \mu, \nu ; \epsilon_{1}, \beta, R\right)=\mathcal{Z}_{2, \text { sing }}^{\text {top }}\left(s, \epsilon_{1}, \beta, R\right) \mathcal{Z}_{2, \text { reg }}^{\text {top }}\left(\lambda, \mu, \nu, \epsilon_{1}, \beta, R\right),
$$

where $\mathcal{Z}_{2, \text { reg }}^{\text {top }}\left(\lambda, \mu, \nu, \epsilon_{1}, \beta, R\right)$ stays finite in the limit $R \rightarrow 0$ and the singular part $\mathcal{Z}_{2 \text {,sing }}^{\text {top }}$ is explicitly given as

$$
\mathcal{Z}_{2, \text { sing }}^{\text {top }}\left(s, \epsilon_{1}, \beta, R\right)=\frac{1}{\mathcal{M}(t / q)} \frac{\left(1-e^{-\epsilon_{1} R}\right)^{\beta^{2} s}}{\left(1-e^{-\epsilon_{1} \beta^{2} R}\right)^{s}}
$$


In order to prove our factorisation (3.4) one may start by rewriting $\mathcal{Z}_{2}^{\text {top }}$ as

$$
\mathcal{Z}_{2}^{\text {top }}=\frac{1}{\mathcal{M}(t / q)} \frac{\left(t^{s+1} ; t\right)_{\infty}(q ; q)_{\infty}}{(t ; t)_{\infty}\left(q^{\beta^{2} s+1} ; q\right)_{\infty}} \frac{\mathcal{M}\left(t^{\lambda} / q\right) \mathcal{M}\left(t^{\mu} / q\right) \mathcal{M}\left(t^{\nu} / q\right) \mathcal{M}(1)}{\mathcal{M}\left(t^{\lambda-s} / q\right) \mathcal{M}\left(t^{\mu-s} / q\right) \mathcal{M}\left(t^{\nu+s} / q\right) \mathcal{M}\left(t^{s}\right)}
$$

The equality between the two expressions for $\mathcal{Z}_{2}^{\text {top }}$ above is easily verified using the functional equations

$$
\mathcal{M}(u t)=(u q, q)_{\infty} \mathcal{M}(u), \quad \mathcal{M}(u q)=(u q, t)_{\infty} \mathcal{M}(u)
$$

The middle factor in (3.6) may be represented in terms of the $q$-Gamma function

$$
\Gamma_{q}(x)=(1-q)^{1-x} \frac{(q ; q)_{\infty}}{\left(q^{x} ; q\right)_{\infty}}
$$

as

$$
\frac{\left(t^{s+1} ; t\right)_{\infty}(q ; q)_{\infty}}{(t ; t)_{\infty}\left(q^{\beta^{2} s+1} ; q\right)_{\infty}}=\frac{(1-q)^{\beta^{2} s}}{(1-t)^{s}} \frac{\Gamma_{q}\left(1+\beta^{2} s\right)}{\Gamma_{t}(1+s)} .
$$

The function $\Gamma_{q}(x)$ is known to have the ordinary Gamma-function $\Gamma(x)$ as its limit $q \rightarrow 1$, so that (3.5) displays a factorisation into a simple singular and a finite part for $R \rightarrow 0$.

We thereby arrive at the factorisation (3.6) with

$$
\mathcal{Z}_{2, \text { reg }}^{\text {top }}=\frac{\Gamma_{q}\left(1+\beta^{2} s\right)}{\Gamma_{t}(1+s)} \mathcal{Z}_{2, \text { bal }}^{\text {top }}, \quad \mathcal{Z}_{2, \text { bal }}^{\text {top }}=\frac{\mathcal{M}\left(t^{\lambda} / q\right) \mathcal{M}\left(t^{\mu} / q\right) \mathcal{M}\left(t^{\nu} / q\right) \mathcal{M}(1)}{\mathcal{M}\left(t^{\lambda-s} / q\right) \mathcal{M}\left(t^{\mu-s} / q\right) \mathcal{M}\left(t^{\nu+s} / q\right) \mathcal{M}\left(t^{s}\right)}
$$

which will be shown to have a finite limit when $R \rightarrow 0$.

\subsection{The limit of the regular part}

In order to show that $\mathcal{Z}_{2 \text {,reg }}^{\text {top }}$ has a finite limit when $R \rightarrow 0$ it is useful to observe that it is a ratio of products of functions that is "perfectly balanced" in the following sense. For any given function $F(x)$ we may call ratios of the form

$$
R(\lambda, \mu, \nu, \delta)=\frac{F(\delta)}{F(\delta-s)} \frac{F(\nu)}{F(\nu-s)} \frac{F(\mu)}{F(\mu+s)} \frac{F(\lambda)}{F(\lambda+s)}
$$

perfectly balanced if $(\lambda, \mu, \nu, \delta)$ satisfy $\mu+\lambda-\nu-\delta=2 s$. If this is the case one easily finds that the function $\tilde{R}(\lambda, \mu, \nu, \delta)$ obtained by replacing the function $F(x)$ in (3.11) by $\tilde{F}(x)=e^{\alpha x^{2}+\beta x} F(x)$ is identically equal to the function $R(\lambda, \mu, \nu, \delta)$. It is easily checked that $\mathcal{Z}_{2, \text { bal }}^{\text {top }} \equiv \mathcal{Z}_{2 \text {,bal }}^{\text {top }}\left(\lambda, \mu, \nu, \beta^{-2}\right)$ is perfectly balanced in this sense. It can be represented as the infinite product

$$
\mathfrak{T}_{s}(\delta, \nu, \mu, \lambda)=\prod_{i, j=0} \mathfrak{t}_{i j}(\delta, \nu, \mu, \lambda)
$$

where $\delta=\beta^{-2}$ and

$$
\mathfrak{t}_{i j}(\delta, \nu, \mu, \lambda)=\vartheta_{i j}^{+}(\delta, s) \vartheta_{i j}^{+}(\nu, s) \vartheta_{i j}^{-}(\mu, s) \vartheta_{i j}^{-}(\lambda, s), \quad \vartheta_{i j}^{ \pm}(\mu, s)=\frac{1-t^{\mu \pm s+i} q^{j}}{1-t^{\mu+i} q^{j}} .
$$


In the limit $R \rightarrow 0$ one finds that

$$
\mathfrak{T}_{s}(\delta, \nu, \mu, \lambda) \rightarrow \mathfrak{R}_{s}(\delta, \nu, \mu, \lambda)=\prod_{i, j=0} \mathfrak{r}_{i j}(\delta, \nu, \mu, \lambda),
$$

where

$$
\mathfrak{r}_{i j}(\delta, \nu, \mu, \lambda)=\varrho_{i j}^{+}(\delta, s) \varrho_{i j}^{+}(\nu, s) \varrho_{i j}^{-}(\mu, s) \varrho_{i j}^{-}(\lambda, s), \quad \varrho_{i j}^{ \pm}(\mu, s)=\frac{j+\beta^{2}(\mu \pm s+i)}{j+\beta^{2}(\mu+i)} .
$$

The crucial point to observe is that the infinite product that defines $\mathfrak{R}_{s}(\delta, \nu, \mu, \lambda)$ is still absolutely convergent thanks to the fact that it is the product of perfectly balanced factors. In order to see this, let us introduce

$$
\Gamma_{\beta}(\beta x)=\Gamma_{2}\left(x \mid 1, \beta^{-2}\right),
$$

with $\Gamma_{2}\left(x \mid \epsilon_{1}, \epsilon_{2}\right)$ defined by the absolutely convergent infinite product

$$
\Gamma_{2}\left(x \mid \epsilon_{1}, \epsilon_{2}\right)=\frac{e^{-\alpha x+\frac{\beta x^{2}}{2}}}{x} \prod_{n_{1}, n_{2} \geq 0}^{\prime} \frac{e^{\frac{x}{\epsilon_{1} n_{1}+\epsilon_{2} n_{2}}-\frac{x^{2}}{2\left(\epsilon_{1} n_{1}+\epsilon_{2} n_{2}\right)^{2}}}}{1+\frac{x}{\epsilon_{1} n_{1}+\epsilon_{2} n_{2}}}, \quad \epsilon_{1}, \epsilon_{2}>0 .
$$

It is then easy to see that

$$
\mathfrak{R}_{s}\left(\beta^{-2}, \nu, \mu, \lambda\right)=\frac{\Gamma_{\beta}\left(\beta^{-1}\right)}{\Gamma_{\beta}\left(\beta^{-1}+\beta s\right)} \frac{\Gamma_{\beta}(\beta \nu)}{\Gamma_{\beta}(\beta(\nu+s))} \frac{\Gamma_{\beta}(\beta \mu)}{\Gamma_{\beta}(\beta(\mu-s))} \frac{\Gamma_{\beta}(\beta \lambda)}{\Gamma_{\beta}(\beta(\lambda-s))} .
$$

Indeed, each factor in the infinite product over $i, j$ obtained by inserting (3.17) into (3.18) is perfectly balanced, making it easy to see that all exponential factors cancel each other, factor by factor. Thus the infinite product defining $\mathfrak{R}_{s}(\delta, \nu, \mu, \lambda)$ is absolutely convergent since the infinite products defining the function $\Gamma_{\beta}(x)$ also have this property.

\subsection{Renormalising the singular part}

One may now be tempted to simply define $\mathcal{Z}_{2}^{4 \mathrm{~d}}$ to be $\mathcal{Z}_{2, \text { reg }}^{\text {top }}\left(\lambda, \mu, \nu, \epsilon_{1}, \beta, R\right)$. However, it is clear that the factorisation (3.4) is ambiguous. One could modify $\mathcal{Z}_{2, \text { sing }}^{\text {top }}$ by multiplying it with an arbitrary function while dividing $\mathcal{Z}_{2, \text { reg }}^{\text {top }}$ by the same function. Additional requirements have to be imposed in order to arrive at an unambiguous definition for $\mathcal{Z}_{2}^{4 \mathrm{~d}}$.

In our case it seems natural to require that the key analytic properties of the function $\mathcal{Z}_{2}^{\text {top }}$ are preserved in the limit. In this regard let us note that the factorisation (3.4) has some special features distinguishing it from other possible factorisations. The singular piece, here recalled for convenience

$$
\mathcal{Z}_{2, \text { sing }}^{\text {top }}\left(s, \epsilon_{1}, \beta, R\right)=\frac{1}{\mathcal{M}(t / q)} \frac{\left(1-e^{-\epsilon_{1} R}\right)^{\beta^{2} s}}{\left(1-e^{-\epsilon_{1} \beta^{2} R}\right)^{s}},
$$

depends (i) on the variables $\lambda, \mu, \nu$ only through the combination $s=\frac{1}{2}\left(\lambda+\mu-\nu-\beta^{-2}\right)$ and (ii) depends on the variable $s$ in a very simple way: the dependence of $\mathcal{Z}_{2 \text {,sing }}^{\text {top }}$ on the variable $s$ is entire analytic, $\mathcal{Z}_{2 \text {,sing }}^{\text {top }}$ is nowhere vanishing as function of $s$, and $\mathcal{Z}_{2 \text {,sing }}^{\text {top }}$ has at most exponential growth. This means that $\log \mathcal{Z}_{2, \text { sing }}^{\text {top }}$ is a linear function. 
Imposing the requirement that these features are preserved in the limit $R \rightarrow 0$ eliminates most of the ambiguities in the renormalisation of $\mathcal{Z}_{2}^{\text {top }}$. The factor $\frac{1}{\mathcal{M}(t / q)}$ does not depend on $s$ at all, while

$$
\frac{\left(1-e^{-\epsilon_{1} R}\right)^{\beta^{2} s}}{\left(1-e^{-\epsilon_{1} \beta^{2} R}\right)^{s}} \sim R^{s\left(\beta^{2}-1\right)} \beta^{-2 s} .
$$

We conclude that the most general renormalised limit $R \rightarrow 0$ satisfying the requirements formulated above is

$$
\lim _{R \rightarrow 0} \eta\left(\rho R^{\beta^{2}}\right)^{-s} \mathcal{M}(t / q) \mathcal{Z}_{2}^{\text {top }}\left(\lambda, \mu, \nu ; \epsilon_{1}, \beta, R\right) .
$$

The factors $\eta$ and $\rho^{-s}$ represent the ambiguity in the definition of the limit that cannot be removed by the requirements above.

Collecting our findings above, introducing the notations

$$
\beta \lambda=2 a_{3}+2 \beta^{-1}-\beta, \quad \beta \mu=-2 a_{2}+\beta, \quad \beta \nu=2 a_{1}+\beta^{-1},
$$

and using the identity

$$
\frac{\Gamma_{\beta}\left(\beta^{-1}\right)}{\Gamma_{\beta}\left(\beta^{-1}+\beta s\right)}=\beta^{-s\left(1+\beta^{2}\right)} \frac{\Gamma(1+s)}{\Gamma\left(1+\beta^{2} s\right)} \frac{\Gamma_{\beta}(\beta)}{\Gamma_{\beta}(\beta+\beta s)}
$$

we arrive at the statement that

$$
\begin{aligned}
\lim _{R \rightarrow 0} \eta\left(\rho R^{\beta^{2}}\right)^{-s} \mathcal{M}(t / q) \mathcal{Z}_{2}^{\text {top }}=\eta\left(\beta^{1+\beta^{2}} \rho\right)^{-s} & \frac{\Gamma_{\beta}(\beta)}{\Gamma_{\beta}(\beta(1+s))} \\
& \times \frac{\Gamma_{\beta}\left(\beta^{-1}+2 a_{1}\right)}{\Gamma_{\beta}\left(\beta^{-1}+2 a_{1}+s \beta\right)} \frac{\Gamma_{\beta}\left(\beta-2 a_{2}\right)}{\Gamma_{\beta}\left((1-s) \beta-2 a_{2}\right)} \frac{\Gamma_{\beta}\left(2 \beta^{-1}-\beta+2 a_{3}\right)}{\Gamma_{\beta}\left(2 \beta^{-1}-(s+1) \beta+2 a_{3}\right)} .
\end{aligned}
$$

With $a_{3}=a_{1}+a_{2}+s \beta$, the above equation becomes

$$
\begin{aligned}
\lim _{R \rightarrow 0} \eta\left(\rho R^{\beta^{2}}\right)^{-s} \mathcal{M}(t / q) \mathcal{Z}_{2}^{\text {top }}=\eta\left(\beta^{1+\beta^{2}} \rho\right)^{-s} \frac{\Gamma_{\beta}(\beta)}{\left.\Gamma_{\beta}\left(\beta+a_{3}-a_{1}-a_{2}\right)\right)} \\
\quad \times \frac{\Gamma_{\beta}\left(\beta^{-1}+2 a_{1}\right)}{\Gamma_{\beta}\left(\beta^{-1}+a_{1}+a_{3}-a_{2}\right)} \frac{\Gamma_{\beta}\left(\beta-2 a_{2}\right)}{\Gamma_{\beta}\left(\beta+a_{1}-a_{2}-a_{3}\right)} \frac{\Gamma_{\beta}\left(2 \beta^{-1}-\beta+2 a_{3}\right)}{\Gamma_{\beta}\left(2 \beta^{-1}-\beta+a_{3}-a_{1}-a_{2}\right)} .
\end{aligned}
$$

Note that the arguments of the double gamma functions can not all be positive when $a_{2}>0$. However, iff $a_{2}<0$ there exists a regime where the arguments of the double gamma functions are all positive. This should be compared with the expression for the topological string partition function in terms of Kähler parameters $Q_{i}, i=1,2,3$. It is manifest in formula (2.22) that the combinations of Kähler parameters appearing in this expression are all positive.

Recall that the calculation above is performed for the case where $q=e^{-\epsilon_{1} R}<1$ and $t=q^{\beta^{2}}<1$. In the regime where $|t|>1$ it is more natural to parameterise $t=q^{-b^{2}}>1$. In this case we obtain

$$
\begin{aligned}
\lim _{R \rightarrow 0} \eta\left(\rho R^{\beta^{2}}\right)^{-s} \mathcal{M}(t / q) \mathcal{Z}_{2}^{\text {top }}= & \eta\left(b^{1-b^{2}} \rho\right)^{-s}\left(\frac{b^{1+b^{2}}}{2 \pi \mathrm{i}} \Gamma\left(-b^{2}\right)\right)^{-s} \frac{\Gamma_{b}(-s b)}{\Gamma_{b}(0)} \\
& \frac{\Gamma_{b}\left(Q+2 \alpha_{1}-s b\right) \Gamma_{b}\left(-2 \alpha_{2}+s b\right) \Gamma_{b}\left(2 \alpha_{3}+2 Q+s b\right)}{\Gamma_{b}\left(Q+2 \alpha_{1}\right) \Gamma_{b}\left(-2 \alpha_{2}\right) \Gamma_{b}\left(2 Q+2 \alpha_{3}\right)} .
\end{aligned}
$$


Comparing to the Liouville three point function we see that the parameter $\rho$ parametrising the ambiguity in the definition of the limit $R \rightarrow 0$ gets related to the parameter called 'cosmological constant' in the Liouville CFT literature.

\subsection{Extending the domain of definition of the $T_{2}$ partition functions}

It will be useful to parameterise the distances between the pairs of legs emanating from the $T_{2}$-vertex vertically, horizontally and diagonally by the variables $p_{1}, p_{2}$ and $p_{3}$, respectively, which is equivalent to the parameterisation

$$
P_{1}^{2}=q^{2 \beta p_{1}}, \quad P_{2}^{2}=q^{2 \beta p_{2}}, \quad P_{3}^{2}=q^{2 \beta p_{3}} .
$$

From the point of view of the world-sheet sigma model it is not unexpected that the definition of the partition function is somewhat ambiguous. As we are dealing with noncompact target spaces having infinite ends indicated by the pairs of parallel external lines in the toric diagram, we expect that naive definitions of the partition functions will be divergent, and need to be regularised. As the cut-off defining the regularisation for a pair of external lines could depend on the parameter describing the asymptotic geometry of the corresponding infinite end, one expects that changes of the cut-offs will change the partition functions by multiplicative factors depending on one of the variables $p_{i}, i=1,2,3$, only. Such factors will be called leg factors in the following. Contributions to the partition functions with mixed dependence on $p_{1}, p_{2}, p_{3}$, on the other hand, are naturally interpreted as contributions coming from a compact region in the target space containing the region where the three legs meet. We'd therefore expect that such contributions are meaningful.

The result for the $T_{2}$ vertex can then be represented as

$$
\mathcal{Z}^{T_{2}}\left(p_{1}, p_{2}, p_{3}\right)=\frac{[\text { Leg factors }]}{G_{\beta}\left(p_{1}+p_{2}+p_{3}\right) \prod_{i=1}^{3} G_{\beta}\left(p_{1}+p_{2}+p_{3}-2 p_{i}\right)},
$$

using the notation

$$
G_{\beta}(x)=\Gamma_{\beta}\left(\frac{1}{2}\left(\beta+\beta^{-1}\right)+x\right) .
$$

This result has been derived under the condition that the Kähler parameters in the $T_{2}$ diagram are all positive, which is equivalent to the inequalities

$$
p_{1}+p_{2}>p_{3}, \quad p_{2}+p_{3}>p_{1}, \quad p_{3}+p_{1}>p_{2} .
$$

Toric CY having sets of parameters $p_{i}, i=1,2,3$ violating any of these inequalities are related to the toric CY described by the $T_{2}$ by flop transitions. The corresponding toric diagrams are depicted in figure 8 .

The subspace $\mathfrak{P}_{\mathbb{R}}$ of the space of parameters of the $T_{2}$ diagrams with real and positive Kähler parameters can be parameterised by the variables $p_{i}, i=1,2,3$. This space is covered exactly once if $p_{i}>0$ for $i=1,2,3$. It breaks up into four chambers $\mathfrak{P}_{\mathbb{R}}^{(i)}$, $i=1,2,3$ and $\mathfrak{P}_{\mathbb{R}}^{(s)}$, defined by the positivity of all Kähler parameters. These chambers are in one-to-one correspondence with the toric diagrams in figure 8 , with $T_{2}^{(s)} \equiv T_{2}$ being the most symmetric one in the middle. Note that the parameter $p_{i}$ describing the width of the 

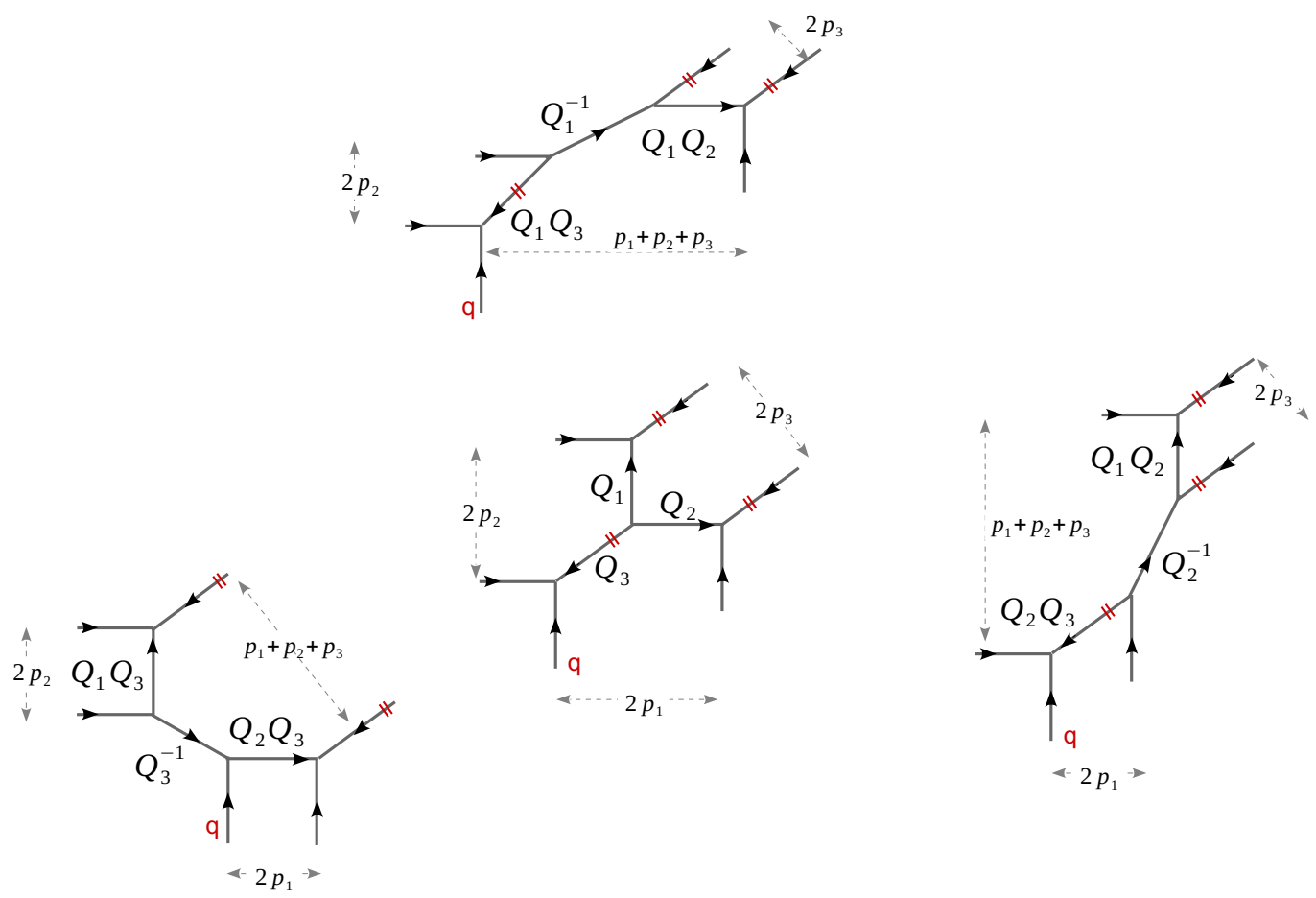

Figure 8. Different $T_{2}$ diagrams related by flops.

corresponding region can grow arbitrarily large in the chamber $\mathfrak{P}_{\mathbb{R}}^{(i)}, i=1,2,3$. One may easily check that the arguments of the functions $G_{\beta}$ appearing in the expressions for $\mathcal{Z}^{T_{2}^{(i)}}$ are always positive within the respective chambers $\mathfrak{P}_{\mathbb{R}}^{(i)}$ for $i=1,2,3, s$.

Each of the new diagrams also has three asymptotic regions bounded by two parallel edges. The distances between these parallel legs are $\sum_{i=1}^{3} p_{i}, 2 p_{i+1}$ and $2 p_{i+2}$ respectively for the diagram $T_{2}^{(i)}$, using the notations $p_{i+3}=p_{i}$. There are known simple rules describing the ratios of the topological string partition functions associated to toric CY related by flop transitions [52-54]. A flop transition describing the continuation from positive to negative values of a Kähler parameter $\log Q_{i}$ will be described by substituting the factor $\mathcal{M}\left(Q_{i}\right)$ in $\mathcal{Z}^{T_{2}^{(i)}}$ by $\mathcal{M}\left(Q_{i}^{-1}\right)$. The corresponding partition functions are thereby found to be

$$
\mathcal{Z}^{T_{2}^{(i)}}=\frac{[\text { Leg factors }]}{\prod_{\epsilon, \epsilon^{\prime}} G_{\beta}\left(p_{i}+\epsilon p_{i+1}+\epsilon^{\prime} p_{i+2}\right)} .
$$

The dependence of the denominator on the variable $p_{i}$ associated to the distinguished asymptotic region is somewhat special in the sense that it is not invariant under $p_{i} \rightarrow-p_{i}$, as is manifestly the case for $j \neq i$.

\section{Integral representation of the $T_{2}$ partition function}

In order to determine the map between the Kähler parameters for the $T_{2}$ vertex and the variables parameterising chiral vertex operators in Liouville conformal field theory, we will now derive an integral representation for the $T_{2}$ partition function $\mathcal{Z}_{2}^{\text {top }}(2.12)$ specialised 
to a discrete family of two-dimensional subspaces inside the three-dimensional parameter space. The specialisation of parameters is known in the CFT literature as the screening condition. We will follow the approach of $[38,39]$, being interested in the precise form of the normalisation factors which had not been determined before. These normalisation factors will turn out to be important for us later.

\subsection{Imposing the specialisation condition to the topological strings}

We begin with the topological string partition function for $T_{2}$ derived in section 2 and written in equation (2.12), which we rewrite here for the convenience of the reader:

$$
\begin{aligned}
& \mathcal{Z}_{2}^{\text {top }}\left(P_{1}, P_{2}, P_{3} ; t, q\right)= \\
& =\frac{\mathcal{M}\left(P_{2}^{2}\right)}{\mathcal{M}\left(\sqrt{\frac{t}{q}} \frac{P_{2} P_{3}}{P_{1}}\right) \mathcal{M}\left(\sqrt{\frac{t}{q}} \frac{P_{1} P_{2}}{P_{3}}\right)} \sum_{\nu}\left(\sqrt{\frac{t}{q}} \frac{P_{1} P_{3}}{P_{2}}\right) \frac{|\nu| \mathcal{N}_{\nu \emptyset}\left(\sqrt{\frac{t}{q}} \frac{P_{2} P_{3}}{P_{1}}\right) \mathcal{N}_{\emptyset \nu}\left(\sqrt{\frac{t}{q}} \frac{P_{1} P_{2}}{P_{3}}\right)}{\mathcal{N}_{\nu \nu}(t / q)} .
\end{aligned}
$$

Following $[38,39]$, an important first step is to observe that if we impose

$$
\sqrt{\frac{t}{q}} \frac{P_{2} P_{3}}{P_{1}}=\frac{t}{q} t^{s}
$$

with $s$ an integer and the length of the partition $\nu$ denoted $N_{\nu}$ that satisfies $N_{\nu}>s$, then the partition function vanishes. Therefore, after substituting (4.2) in (4.1) we can safely replace $N_{\nu}$ by $s$ for the products which exist inside the expansion of the functions $\mathcal{N}_{\mu \nu}$. Then, using equations (A.10) and (A.11) we can recast the topological string partition function of $T_{2}$ as

$$
\begin{aligned}
\mathcal{Z}_{2}^{\text {top }}= & \frac{\prod_{i=1}^{s} \varphi\left(P_{2}^{2} q t^{-i}\right)}{\mathcal{M}\left(v^{-2}\right) \prod_{i=1}^{s} \varphi\left(t^{i}\right)} \sum_{\nu}\left(\frac{P_{1} P_{3}}{v P_{2}}\right)^{|\nu|} \\
& \times \prod_{i=1}^{s} \frac{\varphi\left(P_{2}^{2} v^{2} / y_{\nu, i}\right)}{\varphi\left(P_{2}^{2} v^{2} / y_{\emptyset, i}\right)} \prod_{i=1}^{s} \frac{\varphi\left(1 / y_{\emptyset, i}\right)}{\varphi\left(1 / y_{\nu, i}\right)} \prod_{i, j=1}^{s} \frac{\varphi\left(q^{\nu_{i}-\nu_{j}} t^{j-i}\right)}{\varphi\left(q^{\nu_{i}-\nu_{j}} t^{j-i+1}\right)} \frac{\varphi\left(t^{j-i+1}\right)}{\varphi\left(t^{j-i}\right)}
\end{aligned}
$$

in terms of the quantum dilogarithm $\varphi(x)$ (A.1), with $v=\sqrt{q / t}$ and where the variables $y$ are

$$
y_{\nu, i}=q^{\nu_{i}} t^{s-i}
$$

Further massaging the equation (4.3) we can rewrite the topological strings partition function as

$$
\mathcal{Z}_{2}^{\text {top }}=\frac{\prod_{i=1}^{s} \varphi\left(P_{2}^{2} q t^{-i}\right)}{\mathcal{M}\left(v^{-2}\right) \prod_{i=1}^{s} \varphi\left(t^{i}\right)} \sum_{\nu}\left(\frac{P_{1} P_{3}}{v P_{2}}\right)^{|\nu|} \frac{\mathcal{I}_{m}\left(y_{\nu}\right)}{\mathcal{I}_{m}\left(y_{\emptyset}\right)} \frac{\mathcal{I}_{1,1}\left(y_{\nu}\right)}{\mathcal{I}_{1,1}\left(y_{\emptyset}\right)}
$$

having defined $[38,39]$

$$
\mathcal{I}_{m}\left(y_{\nu}\right)=\prod_{i=1}^{s} \frac{\varphi\left(P_{2}^{2} v^{2} / y_{\nu, i}\right)}{\varphi\left(1 / y_{\nu, i}\right)}, \mathcal{I}_{1,1}\left(y_{\nu}\right)=\prod_{i \neq j=1}^{s} \frac{\varphi\left(q^{\nu_{i}-\nu_{j}} t^{j-i}\right)}{\varphi\left(q^{\nu_{i}-\nu_{j}} t^{j-i+1}\right)}=\prod_{i \neq j=1}^{s} \frac{\varphi\left(y_{\nu, i} / y_{\nu, j}\right)}{\varphi\left(t y_{\nu, i} / y_{\nu, j}\right)}
$$


For the intermediate steps to reproduce this calculation we note that when $s$ is the number of rows of the partition $\nu$, the expansion (A.18) of the Nekrasov function $\mathcal{N}_{\nu \nu}(t / q)$ into quantum dilogarithms allows to obtain $\mathcal{I}_{1,1}(y)$ from

$$
\mathcal{N}_{\nu \nu}(t / q)^{-1}=\mathcal{N}_{\nu \emptyset}\left(t^{1+s} / q\right)^{-1} \mathcal{N}_{\emptyset \nu}\left(t^{1-s} / q\right)^{-1} \frac{\mathcal{I}_{1,1}\left(y_{\nu}\right)}{\mathcal{I}_{1,1}\left(y_{\emptyset}\right)}
$$

Then, the functions $\mathcal{N}_{\nu \emptyset}$ combine to give $\mathcal{I}_{m}(y)$

$$
\frac{\mathcal{N}_{\emptyset \nu}\left(P_{2}^{2} t^{-s}\right)}{\mathcal{N}_{\emptyset \nu}\left(t^{1-s} / q\right)}=\frac{\mathcal{I}_{m}\left(y_{\nu}\right)}{\mathcal{I}_{m}\left(y_{\emptyset}\right)}
$$

\subsection{The matrix integral as a sum of residues}

The summation inside the topological string partition function (4.5) is related to the matrix integral

$$
\mathcal{I}_{2}=\int \frac{d_{q}^{\prime} y_{1}}{y_{1}} \cdots \frac{d_{q}^{\prime} y_{s}}{y_{s}} \prod_{i=1}^{s} y_{i}^{\zeta+1} \mathcal{I}_{1,1}(y) \mathcal{I}_{m}(y),
$$

where the parameter $\zeta$ is defined by

$$
q^{\zeta+1}=\sqrt{\frac{t}{q}} \frac{P_{1} P_{3}}{P_{2}},
$$

and the integrals $\int d_{q}^{\prime} y_{1} \cdots d_{q}^{\prime} y_{s} \prod_{i=1}^{s} y_{i}^{-1}$ are variants of the Jackson integral defined for meromorphic functions $M(y)$ of $s$ variables $y=\left(y_{1}, \ldots, y_{s}\right)$ as a sum over residues

$$
\int \frac{d_{q}^{\prime} y_{1}}{y_{1}} \cdots \frac{d_{q}^{\prime} y_{s}}{y_{s}} M(y):=(2 \pi \mathrm{i})^{s} \sum_{\substack{R_{1}, \ldots, R_{s} \in \mathbb{N} \\ R_{1}>R_{2}>\cdots>R_{s}}} \operatorname{Res}_{y=y_{R}} M(y) .
$$

Summation over residues. To understand the relation between the integral (4.9) and its expression as a sum over residues precisely, we discuss the pole structure of the integrand, noting that it parallels to a large extent the analysis of $[38,39]$. We nevertheless review this here for clarity and completeness. Assuming a radial ordering of the poles $\left|y_{i}\right|<\left|y_{i+1}\right|$, these originate from:

- $\mathcal{I}_{m}(y)$ : outermost pole $y_{s}=q^{\nu_{s}}$

- $\mathcal{I}_{1,1}(y)$ : poles organised by a partition $\nu$, with $y_{i}=q^{\nu_{i}} t^{s-i}, 1 \leq i<s$.

The reasoning behind this statement is as follows. The poles of the function $\mathcal{I}_{m}(y)$ are located at $y_{i}=q^{m}, m \in \mathbb{N}$ while, in the regime where $|q|,|t|<1$, those of the function $\mathcal{I}_{1,1}(y)$ satisfy $y_{i} / y_{i+1}=q^{n} t$. The outermost pole $y_{s}$ therefore has to belong to $\mathcal{I}_{m}(y)$, since it would otherwise be inconsistent with the radial ordering. Having established this fact, no other poles can originate from $\mathcal{I}_{m}(y)$ because any such singularities would be cancelled by zeros of $\mathcal{I}_{1,1}(y)$. Consequently, all of the remaining poles belong to the function $\mathcal{I}_{1,1}(y)$ and $y_{s-1} / y_{s}=q^{n} t$ implies $y_{s-1}=q^{\nu_{s-1}} t$, with $\nu_{s-1}>\nu_{s}$. Iterating this logic, we find the remaining poles $y_{i}=q^{\nu_{i}} t^{s-i}$, the set of which is therefore labelled by a Young tableau $\nu$

$$
y_{\nu}=\left(q^{\nu_{1}} t^{s-1}, \ldots, q^{\nu_{s-1}} t, q^{\nu_{s}}\right) \text {. }
$$


The matrix integral (4.9) is therefore defined through the sum

$$
\mathcal{I}_{2}=(2 \pi i)^{s}\left(\operatorname{Res}_{\emptyset}\right) \sum_{\nu} q^{(\zeta+1)|\nu|} \frac{\mathcal{I}_{1,1}\left(y_{\nu}\right)}{\mathcal{I}_{1,1}\left(y_{\emptyset}\right)} \frac{\mathcal{I}_{m}\left(y_{\nu}\right)}{\mathcal{I}_{m}\left(y_{\emptyset}\right)},
$$

where the coefficient

$$
\operatorname{Res}_{\emptyset}=t^{\frac{1}{2} s(s-1)(\zeta+1)}\left(\frac{\varphi(t)}{\varphi(q)}\right)^{s} \prod_{i=1}^{s} \frac{\varphi\left(P_{2}^{2} q t^{i-s-1}\right)}{\varphi\left(t^{i}\right)}
$$

is obtained by evaluating the integral (4.9) with $\nu$ empty. One thus finds the same summation over Young diagrams on the right hand side of both equations (4.5) for the $T_{2}$ topological string partition function and (4.13) for the integral formulation.

\subsection{The free field representation for $q$-Liouville}

In this section we briefly discuss the relation between the matrix integral (4.9) and mathematical objects called $q$-deformed Virasoro conformal blocks which represent a deformation of the integrals representing conformal blocks in conformal field theory. The $q$-deformation of a three point conformal block on $\mathbb{C P}^{1} \backslash\{0,1, \infty\}$ with primary fields $V_{a_{1}}, V_{a_{2}}$ and $V_{a_{3}}$ inserted at the locations of the punctures and with $\beta s=a_{3}-a_{2}-a_{1}$, for example, can be written as

$$
\mathcal{B}_{q-\text { Liouv }} \equiv \oint d y \mathcal{I}_{q-\text { Liouv }}
$$

with the integrand

$$
\mathcal{I}_{q-\text { Liouv }}=\prod_{i=1}^{s} y_{i}^{2 \beta a_{1}}\left\langle S\left(y_{i}\right) V_{a_{2}}(1)\right\rangle \prod_{j>i}^{s}\left\langle S\left(y_{j}\right) S\left(y_{i}\right)\right\rangle,
$$

where without any loss of generality we send $z_{3} \rightarrow \infty$. The two point function of $q$-Liouville between a primary field and a screening current is $[55,56]$

$$
\left\langle S\left(y_{i}\right) V_{a_{2}}(z)\right\rangle=y_{i}^{2 \beta a_{2}} \frac{\varphi\left(q^{-2 \beta a_{2}} / y_{i}\right)}{\varphi\left(1 / y_{i}\right)},
$$

while that between two screening currents is $[55,56]$

$$
\left\langle S\left(y_{j}\right) S\left(y_{i}\right)\right\rangle=\left(y_{j}\right)^{2 \beta^{2}} \frac{\varphi\left(y_{i} / y_{j}\right) \varphi\left(q t^{-1} y_{i} / y_{j}\right)}{\varphi\left(q y_{i} / y_{j}\right) \varphi\left(t y_{i} / y_{j}\right)}, \quad\left|y_{i}\right|<\left|y_{j}\right|, i<j .
$$

One should note that a different definition of the two point function (4.18) has been used in the references [38, 39].

We may already observe that the factor $\mathcal{I}_{m}$ in (4.6) is proportional to the two-point function (4.17) provided the parameter $P_{2}$ is related to $a_{2}$ by $P_{2}^{2}=\frac{t}{q} q^{-2 \beta a_{2}}$. To also reach agreement between the ratio of quantum dilogarithms in the two point function (4.18) and the function $\mathcal{I}_{1,1}$ inside (4.6), it is necessary to rewrite (4.18) using the following function

$$
\vartheta_{q}\left(\frac{y_{i}}{y_{j}}, 1-\beta^{2}\right)=\left(\frac{y_{j}}{y_{i}}\right)^{\beta^{2}} \frac{\varphi\left(q t^{-1} y_{i} / y_{j}\right)}{\varphi\left(q y_{i} / y_{j}\right)} \frac{\varphi\left(t y_{j} / y_{i}\right)}{\varphi\left(y_{j} / y_{i}\right)} .
$$


The function $\vartheta_{q}(x, \kappa)$ is a quasi-constant, meaning that it does not depend on multiplicative shifts of the argument by $q$,

$$
\vartheta_{q}(x, \kappa)=\vartheta_{q}(q x, \kappa) .
$$

If we now introduce the short-hand grouping all quasi-constants

$$
\vartheta_{q}(y, s)=\prod_{j>i}^{s} \vartheta_{q}\left(\frac{y_{i}}{y_{j}}, 1-\beta^{2}\right)
$$

gather the contributions of all factors $y_{i}$ into $y_{i}^{\zeta}$ with

$$
\zeta=2 \beta\left(a_{1}+a_{2}\right)+\beta^{2}(s-1)
$$

then the three point conformal block $\mathcal{B}_{q-\text { Liouv }}$ in the free field representation with $z_{1}=$ $0, z_{2}=1$ and $z_{3}=\infty$ can be written proportional to the matrix model integral (4.9)

$$
\begin{aligned}
\mathcal{B}_{q-\text { Liouv }} & =\int d_{q}^{\prime} y_{1} \cdots d_{q}^{\prime} y_{s} \prod_{i=1}^{s} y_{i}^{\zeta} \mathcal{I}_{m}(y) \mathcal{I}_{1,1}(y) \vartheta_{q}(y, s) \\
& =\vartheta_{q}(y, s) \mathcal{I}_{2}\left(a_{1}, a_{2}, s ; \beta\right)
\end{aligned}
$$

The quasi-constant factors, through their invariance with respect to the multiplicative factor $q$, can all be pulled outside of the integral in equation (4.23). Furthermore, the power $\zeta$ appearing in this equation, together with the identity (4.10) and the specialisation (4.2), fixes the remaining entries of the dictionary between the topological string parameters $P_{1}$ and $P_{3}$ and the conformal field theory momenta $a_{1}$ and $a_{2}$ to be

$$
P_{1}^{2}=\frac{q}{t} q^{2 \beta a_{1}}, \quad P_{2}^{2}=\frac{t}{q} q^{-2 \beta a_{2}}, \quad P_{3}^{2}=\frac{q}{t} q^{2 \beta a_{3}}, \quad t=q^{\beta^{2}},
$$

where $a_{3}=a_{1}+a_{2}+s \beta .^{5}$

Given the relations (4.24) between the relevant parameters the relation between the $T_{2}$ topological strings partition function (4.5) and the matrix model integral (4.9) is

$$
\mathcal{I}_{2}\left(a_{1}, a_{2}, s ; \beta\right)=(2 \pi i)^{s} t^{\frac{1}{2} s(s-1)(\zeta+1)}\left(\frac{\varphi(t)}{\varphi(q)}\right)^{s} \mathcal{M}(t / q) \mathcal{Z}_{2}^{\mathrm{top}}\left(P_{1}, P_{2}, P_{3} ; t, q\right)
$$

We will next see that the integral $\mathcal{I}_{2}\left(a_{1}, a_{2}, s ; \beta\right)$ has a well-defined limit $q \rightarrow 1$.

\section{$5 \quad$ The $q \rightarrow 1$ limit of the Matrix integral}

Having established the relation between the topological string partition function and the matrix integral, we wish to take the $q \rightarrow 1$ limit of the latter. Looking at definitions (4.6), (4.9), as well as (A.24), one will notice that the function $\varphi(z)$ representing the main building block of the integrand diverges for $q \rightarrow 1$, and that the summation over residues defining $\mathcal{I}_{2}$ does not have an obvious limit.

\footnotetext{
${ }^{5}$ We remark that the dictionary (4.24) fully agrees with equations (3.1) and (3.22) in section 3.
} 
Our strategy so far to resolve this issue has been to first rewrite the sum (4.13) as a variant of the Jackson integral, then recast this in terms of combinations of functions $\varphi(z)$ which are known to have a well-defined limit when $q \rightarrow 1$. Now we will rewrite the integrand using the quasi-constants $\vartheta_{q}(x, s)$ defined in equation (4.19) such that all the singularities are contained inside a product of quasi-constant terms. As a result we will discover the relation to the usual Selberg integral.

\subsection{A simple example}

Let us first consider a simple example. We are ultimately interested in evaluating the $q \rightarrow 1^{-}$limit of integrals of the form

$$
\mathcal{I}_{q}=\int_{\mathcal{C}} d x \mathcal{R}(x ; s, t), \quad \mathcal{R}(x ; s, t)=x^{t+s-2} \frac{\varphi\left(q^{1-s} / x\right)}{\varphi(1 / x)},
$$

where $\mathcal{C}$ is a contour starting and ending at 0 , encircling the poles of the integrand in the interval $(0,1)$ in the counterclockwise direction. The integrand (5.1) has poles at $x=q^{n}$, $n \in \mathbb{N}$. The integral $\mathcal{I}_{q}$ can therefore be evaluated as a sum of the residues

$$
\mathcal{I}_{q}=2 \pi \mathrm{i} \sum_{n=0}^{\infty} \mathcal{R}_{n}(s, t), \quad \mathcal{R}_{n}(s, t):=\operatorname{Res}_{x=q^{n}} \mathcal{R}(x ; s, t) .
$$

For what follows we find it useful to introduce a variant of the Jacobi triple product function

$$
\vartheta_{q}(z):=\varphi(z) \varphi(q / z) \varphi(q)=(1-z) \prod_{n=1}\left(1-z q^{n}\right)\left(1-z^{-1} q^{n}\right)\left(1-q^{n}\right)
$$

which we may use to rewrite the integrand $\mathcal{R}(x ; s, t)$ of $\mathcal{I}_{q}$ as

$$
\frac{\varphi\left(q^{1-s} / x\right)}{\varphi(1 / x)}=\frac{\varphi(q x)}{\varphi\left(q^{s} x\right)} \frac{\vartheta_{q}\left(q^{s} x\right)}{\vartheta_{q}(q x)} .
$$

The function $\vartheta_{q}(z)$ allows us to represent $\mathcal{I}_{q}$ as

$$
\mathcal{I}_{q}=2 \pi \mathrm{i} \sum_{n=0}^{\infty} \rho(s) \mathcal{R}_{n}^{\prime}(s, t), \quad \mathcal{R}_{n}^{\prime}(s, t):=\left[x^{t-1} \frac{\varphi(q x)}{\varphi\left(q^{s} x\right)}\right]_{x=q^{n}}
$$

and where $\rho(s)$ is given by

$$
\rho(s)=\operatorname{Res}_{x=q^{n}} x^{s-1} \frac{\vartheta_{q}\left(q^{s} x\right)}{\vartheta_{q}(q x)}=q^{n} \frac{\vartheta_{q}\left(q^{s}\right)}{(q ; q)_{\infty}^{3}} .
$$

It follows that the integral (5.1) can be rewritten in terms of an integral $\mathcal{I}_{q}^{\prime}$

$$
\mathcal{I}_{q}=\frac{2 \pi \mathrm{i}}{1-q} \frac{\vartheta_{q}\left(q^{s}\right)}{(q ; q)_{\infty}^{3}} \mathcal{I}_{q}^{\prime}, \quad \mathcal{I}_{q}^{\prime}=\int_{0}^{1} d_{q} x x^{t-1} \frac{(q x ; q)_{\infty}}{\left(q^{s} x ; q\right)_{\infty}},
$$

where $\mathcal{I}_{q}^{\prime}$ can be evaluated as Jacksons integral

$$
\int_{0}^{1} \frac{d_{q} x}{x} f(x)=(1-q) \sum_{k=0}^{\infty} f\left(q^{k}\right) .
$$




\subsection{The matrix integral}

We now return to the specific case in which we are interested, equation (4.9). Identity (4.19) allows us to rewrite the integral (4.9) as

$$
\begin{aligned}
\mathcal{I}_{2}=\int d_{q}^{\prime} y_{1} \cdots d_{q}^{\prime} y_{s} & \prod_{i=1}^{s} y_{i}^{\zeta} \frac{\varphi\left(q y_{i}\right)}{\varphi\left(t y_{i} / P_{2}^{2}\right)} \vartheta_{q}\left(y_{i}, 1+2 \beta a_{2}\right)\left(y_{i}\right)^{-2 \beta a_{2}} \\
& \prod_{j>i}^{s} \frac{\varphi\left(y_{i} / y_{j}\right)}{\varphi\left(t y_{i} / y_{j}\right)} \frac{\varphi\left(v^{2} y_{i} / y_{j}\right)}{\varphi\left(q y_{i} / y_{j}\right)} \vartheta_{q}\left(\frac{y_{i}}{t y_{j}}, 1+\beta^{2}\right)\left(\frac{y_{i}}{t y_{j}}\right)^{-\beta^{2}},
\end{aligned}
$$

where we recall that the integrals $\int d_{q}^{\prime} y_{1} \cdots d_{q}^{\prime} y_{s} \prod_{i=1}^{s} y_{i}^{-1}$ are variants of the Jackson integral (4.11). We also recall that as explained in section 4.2 we have assumed the radial ordering of poles $\left|y_{i}\right|<\left|y_{i+1}\right|$, which are labelled by a partition $\nu$

$$
y_{\nu}=\left\{y_{1}, y_{2}, \ldots, y_{s-1}, y_{s}\right\}_{\nu}=\left\{t^{s-1} q^{\nu_{1}}, t^{s-2} q^{\nu_{2}}, \ldots, t q^{\nu_{s-1}}, q^{\nu_{s}}\right\} .
$$

Since quasi-constants are independent of multiplicative shifts of the argument by $q$, they can be factored out of the integrand and $\mathcal{I}_{2}$ in equation $(5.9)$ becomes

$$
\begin{array}{r}
\mathcal{I}_{2}=t^{\frac{s(s-1)}{2} \beta^{2}}\left(\frac{2 \pi \mathrm{i}}{1-q}\right)^{s} \operatorname{Eval}_{y=y_{\nu_{\emptyset}}}\left[\prod_{i=1}^{s-1} \vartheta_{q}\left(y_{i}, 1+2 \beta a_{2}\right) \prod_{j-i \geq 2}^{s} \vartheta_{q}\left(\frac{y_{i}}{t y_{j}}, 1+\beta^{2}\right)\right] \\
\operatorname{Res}_{y_{s}=1} \vartheta_{q}\left(y_{s}, 1+2 \beta a_{2}\right) \prod_{i=1}^{s-1} \operatorname{Res}_{y_{i}=t y_{i+1}} \vartheta_{q}\left(\frac{y_{i}}{t y_{i+1}}, 1+\beta^{2}\right) \\
\int d_{q}^{\prime} y_{1} \cdots d_{q}^{\prime} y_{s} \prod_{i=1}^{s} y_{i}^{2 \beta^{2}(i-1)+2 \beta a_{1}} \frac{\varphi\left(q y_{i}\right)}{\varphi\left(t y_{i} / P_{2}^{2}\right)} \prod_{i<j}^{s} \frac{\varphi\left(y_{i} / y_{j}\right)}{\varphi\left(t y_{i} / y_{j}\right)} \frac{\varphi\left(v^{2} y_{i} / y_{j}\right)}{\varphi\left(q y_{i} / y_{j}\right)} .
\end{array}
$$

The residues can be evaluated, as we show in appendix B, and in particular using the definition (4.19), and we obtain

$$
\operatorname{Res}_{y_{i}=t y_{i+1}} \vartheta_{q}\left(\frac{y_{i}}{t y_{i+1}}, 1+\beta^{2}\right)=\frac{\vartheta_{q}\left(q^{-\beta^{2}}\right)}{\varphi(q)^{3}}
$$

and

$$
\operatorname{Res}_{y_{s}=1} \vartheta_{q}\left(y_{s}, 1+2 \beta a_{2}\right)=\frac{\vartheta_{q}\left(q^{-2 \beta a_{2}}\right)}{\varphi(q)^{3}} .
$$

Such ratios have a well defined $q \rightarrow 1$ limit

$$
\lim _{q \rightarrow 1} \frac{\varphi\left(q^{\alpha_{1}} x\right)}{\varphi\left(q^{\alpha_{2}} x\right)}=(1-x)^{\alpha_{2}-\alpha_{1}}, \quad \frac{\vartheta_{q}\left(q^{s}\right)}{\vartheta_{q}\left(q^{t}\right)} \stackrel{q \rightarrow 1}{\longrightarrow} \frac{\sin (\pi s)}{\sin (\pi t)}, \quad \frac{2 \pi \mathrm{i}}{1-q} \frac{\vartheta_{q}\left(q^{s}\right)}{\varphi(q)^{3}} \stackrel{q \rightarrow 1}{\longrightarrow} 2 \mathrm{i} \sin (\pi s),
$$

see appendix A.1 and appendix B for details. Thus the integral (5.11) becomes

$$
\begin{aligned}
\lim _{q \rightarrow 1} \mathcal{I}_{2} \longrightarrow & \prod_{k=0}^{s-1} 2 \mathrm{i} \sin \left(\pi \beta\left(2 a_{2}+k \beta\right)\right) \\
& \int_{0}^{1} d y_{s} \int_{0}^{y_{s}} d y_{s-1} \ldots \int_{0}^{y_{2}} d y_{1} \prod_{i=1}^{s} y_{i}^{2 \beta a_{1}}\left(1-y_{i}\right)^{2 \beta a_{2}} \prod_{i<j}\left(y_{j}-y_{i}\right)^{2 \beta^{2}}
\end{aligned}
$$


Obtaining the $\prod_{k=0}^{s-1} \sin \left(\pi \beta\left(2 a_{2}+k \beta\right)\right)$ prefactor in front of the Selberg integral is an important point of our paper as it reflects a particular choice of basis in the space of conformal blocks. This choice is not particularly spectacular for the space of Virasoro three point conformal blocks, since this is one dimensional, completely fixed via Ward identities. The real importance comes from the implication for the higher rank $T_{N}$ theories. In this context the $T_{N}$ topological strings partition function is expected to correspond to a particular choice of basis for the space of $W_{N}$ three point conformal blocks, which is infinite dimensional for $N \geq 3$.

\subsection{The matrix integral as a Selberg integral}

We can finally express the limit of $\mathcal{I}_{2}$ in terms of $\Gamma$-functions through the Selberg integral

$$
\lim _{q \rightarrow 1} \mathcal{I}_{2}=\prod_{k=0}^{s-1} 2 \mathrm{i} \sin \left(\pi \beta\left(2 a_{2}+k \beta\right)\right) \mathcal{I}_{\text {Sel }}\left(1+2 \beta a_{1}, 1+2 \beta a_{2}, \beta^{2}\right),
$$

using the definition

$$
\begin{aligned}
\mathcal{I}_{\mathrm{Sel}}\left(1+2 \beta a_{1}, 1+2 \beta a_{2}, \beta^{2}\right)= \\
\quad=\int_{0}^{1} d y_{s} \int_{0}^{y_{s}} d y_{s-1} \ldots \int_{0}^{y_{2}} d y_{1} \prod_{i=1}^{s} y_{i}^{2 \beta a_{1}}\left(1-y_{i}\right)^{2 \beta a_{2}} \prod_{i<j}\left(y_{j}-y_{i}\right)^{2 \beta^{2}} \\
=\prod_{j=0}^{s-1} \frac{\Gamma\left(1+2 \beta a_{1}+j \beta^{2}\right) \Gamma\left(1+2 \beta a_{2}+j \beta^{2}\right) \Gamma\left((j+1) \beta^{2}\right)}{\Gamma\left(2+2 \beta\left(a_{1}+a_{2}\right)+(s-1+j) \beta^{2}\right) \Gamma\left(\beta^{2}\right)}
\end{aligned}
$$

Note that this integral is convergent for $a_{1}, a_{2}$ and $\beta$ real and positive. Furthermore, using the shift identities of the $\Gamma$ function to suppress the products in equation (5.16) by rewriting this in terms of the double gamma function $\Gamma_{\beta}$ :

$$
\frac{\pi}{\sin (\pi x)}=\Gamma(1-x) \Gamma(x)
$$

and

$$
\frac{\Gamma_{\beta}(x+\beta)}{\Gamma_{\beta}(x)}=\sqrt{2 \pi} \beta^{\frac{1}{2}-\beta x} \Gamma^{-1}(\beta x),
$$

this becomes

$$
\begin{aligned}
& \mathcal{I}_{\text {Sel }}\left(1+2 \beta a_{1}, 1+2 \beta a_{2}, \beta^{2}\right)=\left(\frac{2 \pi \mathrm{i}}{\beta^{\beta^{2}-1} \Gamma\left(\beta^{2}\right)}\right)^{s} \frac{\Gamma_{\beta}(\beta)}{\Gamma_{\beta}\left(2 \beta^{-1}-\beta+a_{1}+a_{2}+a_{3}\right)} \\
& \frac{\Gamma_{\beta}\left(\beta^{-1}+2 a_{1}\right) \Gamma_{\beta}\left(\beta^{-1}+2 a_{2}\right) \Gamma_{\beta}\left(2 \beta^{-1}-\beta+2 a_{3}\right)}{\Gamma_{\beta}\left(\beta^{-1}+a_{3}+a_{1}-a_{2}\right) \Gamma_{\beta}\left(\beta^{-1}+a_{3}+a_{2}-a_{1}\right) \Gamma_{\beta}\left(\beta+a_{3}-a_{1}-a_{2}\right)},
\end{aligned}
$$

recalling the condition $a_{3}=a_{1}+a_{2}+s \beta$. We can further make the following observations.

- We can extend the domain of definition from integer $s$ to $s \in \mathbb{C}$.

- The arguments of the double gamma functions are all positive if $a_{i}>0$ for $i=1,2,3$ and $s>0$. 
Taking into account the factor $\prod_{k=0}^{s-1} \sin \left(\pi \beta\left(2 a_{2}+k \beta\right)\right)$ in front of the Selberg integral (5.16), this equation becomes

$$
\begin{aligned}
\lim _{q \rightarrow 1} \mathcal{I}_{2}= & \left(\frac{2 \pi \mathrm{i}}{\beta^{\beta^{2}-1} \Gamma\left(\beta^{2}\right)}\right)^{s} \frac{\Gamma_{\beta}(\beta)}{\Gamma_{\beta}((s+1) \beta)} \\
& \frac{\Gamma_{\beta}\left(\beta^{-1}+2 a_{1}\right)}{\Gamma_{\beta}\left(\beta^{-1}+a_{1}-a_{2}+a_{3}\right)} \frac{\Gamma_{\beta}\left(\beta-2 a_{2}\right)}{\Gamma_{\beta}\left(a_{1}-a_{2}-a_{3}+\beta\right)} \frac{\Gamma_{\beta}\left(2 \beta^{-1}-\beta+2 a_{3}\right)}{\Gamma_{\beta}\left(2 \beta^{-1}-\beta+a_{1}+a_{2}+a_{3}\right)} .
\end{aligned}
$$

Note. So far we have been considering the regime where $|q|<1,|t|<1$ corresponding to CFT with central charge $c=1-6\left(\beta-\beta^{-1}\right)^{2}$ if $t=q^{\beta^{2}}$. Another interesting regime is $|t|>1$ and $|q|<1$. Similar arguments as used above would lead to

$$
\begin{aligned}
\mathcal{Z}_{\text {Liou }}\left(\alpha_{1}, \alpha_{2}, \alpha_{3}\right)= & \left(\frac{b^{1+b^{2}}}{2 \pi \mathrm{i}} \Gamma\left(-b^{2}\right)\right)^{-s} \frac{\Gamma_{b}(-s b)}{\Gamma_{b}(0)} \\
& \frac{\Gamma_{b}\left(Q+2 \alpha_{1}-s b\right) \Gamma_{b}\left(-2 \alpha_{2}+s b\right) \Gamma_{b}\left(2 \alpha_{3}+2 Q+s b\right)}{\Gamma_{b}\left(Q+2 \alpha_{1}\right) \Gamma_{b}\left(-2 \alpha_{2}\right) \Gamma_{b}\left(2 Q+2 \alpha_{3}\right)} .
\end{aligned}
$$

The regime $|q|<1,|t|<1$ is related to the CFT called Generalised Minimal Models in [57], see also [58]. The parameters of the two regimes are related by analytic continuation,

$$
b=-\mathrm{i} \beta, \quad \alpha=\mathrm{i} a, \quad Q=\mathrm{i} q,
$$

provided that $b$ is the parameter giving the central charge of Liouville field theory as $c_{\text {Liouv }}=1+6\left(b+b^{-1}\right)^{2}$.

\section{Comparison with the strip vertex}

We have so far focused on the $T_{2}$ vertex. There exists however another important building block which has been well studied in literature with which the $T_{2}$ should be compared. This is usually referred to as the strip geometry [41], which we review below. One version of the strip is depicted in figure 9 , other variants of the strip being related to this by flop transitions. From the point of view of gauge theory, its partition function is well known to give the partition function of $\mathrm{SU}(2) \times \mathrm{SU}(2)$ bifundamental hypermultiplets.

We will begin by comparing the results of the topological vertex computations for the respective regions in the parameter space. It will turn out, however, that the strip diagrams do not allow us to cover the full parameter space of the $T_{2}$ vertex and its relatives obtained by flop transitions. For the regions of the parameter space for which both the $T_{2}$ and the strip vertex can be used we will find agreement.

This agreement is not unexpected. The toric CY associated to the strip and $T_{2}$ have the same mirror manifolds, indicating that the partition functions associated to these two diagrams should be related. In the second half of this section we will briefly discuss two more direct ways for understanding the relation between the $T_{2}$ vertex and strip from the point of view of the B-model, using the relation between geometric transitions and matrix models on the one hand, and recent results on the topological recursion for the case of our interest on the other hand. 


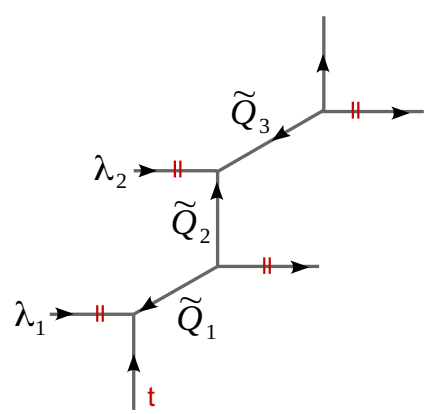

Figure 9. The strip geometry, with the assignment of Kähler parameters, the choice of preferred direction and a pair of Young tableaux decorations on external legs.

\subsection{Topological vertex computation}

The topological strings partition function for the strip geometry has the property that all the sums over Young diagrams can be performed and it can be written in product form. Starting with

$$
\mathcal{Z}_{\text {strip }, \vec{\lambda}}^{\text {top }}\left(\tilde{Q}_{1}, \tilde{Q}_{2}, \tilde{Q}_{3} ; t, q\right)=\sum_{\nu} \prod_{i=1}^{3}\left(-\tilde{Q}_{i}\right)^{\left|\nu_{i}\right|} C_{\nu_{1}^{t} \emptyset \lambda_{1}^{t}}(q, t) C_{\nu_{1} \nu_{2} \emptyset}(t, q) C_{\nu_{3}^{t} \nu_{2}^{t} \lambda_{2}^{t}}(q, t) C_{\nu_{3} \emptyset \emptyset}(t, q)
$$

keeping two of the Young diagrams decorating the external legs non-empty, this equation can be brought to the form

$$
\begin{gathered}
\mathcal{Z}_{\text {strip }, \vec{\lambda}}^{\text {top }}\left(\tilde{Q}_{1}, \tilde{Q}_{2}, \tilde{Q}_{3} ; t, q\right)=t^{\frac{\left\|\lambda_{1}^{t}\right\|^{2}+\left\|\lambda_{2}^{t}\right\|^{2}}{2}} \tilde{Z}_{\lambda_{1}^{t}}(q, t) \tilde{Z}_{\lambda_{2}^{t}}(q, t) \\
\frac{\mathcal{M}\left(\tilde{Q}_{1} \tilde{Q}_{2} ; t, q\right) \mathcal{M}\left(\tilde{Q}_{2} \tilde{Q}_{3} \frac{t}{q} ; t, q\right)}{\mathcal{M}\left(\tilde{Q}_{1} \sqrt{\frac{t}{q}} ; t, q\right) \mathcal{M}\left(\tilde{Q}_{2} \sqrt{\frac{t}{q}} ; t, q\right) \mathcal{M}\left(\tilde{Q}_{3} \sqrt{\frac{t}{q}} ; t, q\right) \mathcal{M}\left(\tilde{Q}_{1} \tilde{Q}_{2} \tilde{Q}_{3} \sqrt{\frac{t}{q}} ; t, q\right)} \\
\frac{\mathcal{N}_{\lambda_{1} \emptyset}\left(\tilde{Q}_{1} \sqrt{\frac{t}{q}} ; t, q\right) \mathcal{N}_{\emptyset \lambda_{2}}\left(\tilde{Q}_{2} \sqrt{\frac{t}{q}} ; t, q\right) \mathcal{N}_{\lambda_{2} \emptyset}\left(\tilde{Q}_{3} \sqrt{\frac{t}{q}} ; t, q\right) \mathcal{N}_{\lambda_{1} \emptyset}\left(\tilde{Q}_{1} \tilde{Q}_{2} \tilde{Q}_{3} \sqrt{\frac{t}{q}} ; t, q\right)}{\mathcal{N}_{\lambda_{1} \lambda_{2}}\left(\tilde{Q}_{1} \tilde{Q}_{2} ; t, q\right)} .
\end{gathered}
$$

Setting then all of the external legs' Young tableaux to $\emptyset$, see for example also [40], this partition function reduces to

$$
\mathcal{Z}_{\text {strip }}^{\text {top }}\left(\tilde{Q}_{1}, \tilde{Q}_{2}, \tilde{Q}_{3} ; t, q\right)=\frac{\mathcal{M}\left(\tilde{Q}_{1} \tilde{Q}_{2}\right) \mathcal{M}\left(\tilde{Q}_{2} \tilde{Q}_{3} \frac{t}{q}\right)}{\mathcal{M}\left(\tilde{Q}_{1} \sqrt{\frac{t}{q}}\right) \mathcal{M}\left(\tilde{Q}_{2} \sqrt{\frac{t}{q}}\right) \mathcal{M}\left(\tilde{Q}_{3} \sqrt{\frac{t}{q}}\right) \mathcal{M}\left(\tilde{Q}_{1} \tilde{Q}_{2} \tilde{Q}_{3} \sqrt{\frac{t}{q}}\right)} .
$$

We may compare this equation to its $T_{2}$ counterpart, whose topological strings partition function was given in product form in equation $(2.22)$ as

$$
\mathcal{Z}_{2}^{\text {top }}\left(Q_{1}, Q_{2}, Q_{3} ; t, q\right)=\frac{\mathcal{M}\left(Q_{1} Q_{2}\right) \mathcal{M}\left(Q_{1} Q_{3} \frac{t}{q}\right) \mathcal{M}\left(Q_{2} Q_{3}\right)}{\mathcal{M}\left(Q_{1} \sqrt{\frac{t}{q}}\right) \mathcal{M}\left(Q_{2} \sqrt{\frac{t}{q}}\right) \mathcal{M}\left(Q_{3} \sqrt{\frac{t}{q}}\right) \mathcal{M}\left(Q_{1} Q_{2} Q_{3} \sqrt{\frac{t}{q}}\right)} .
$$



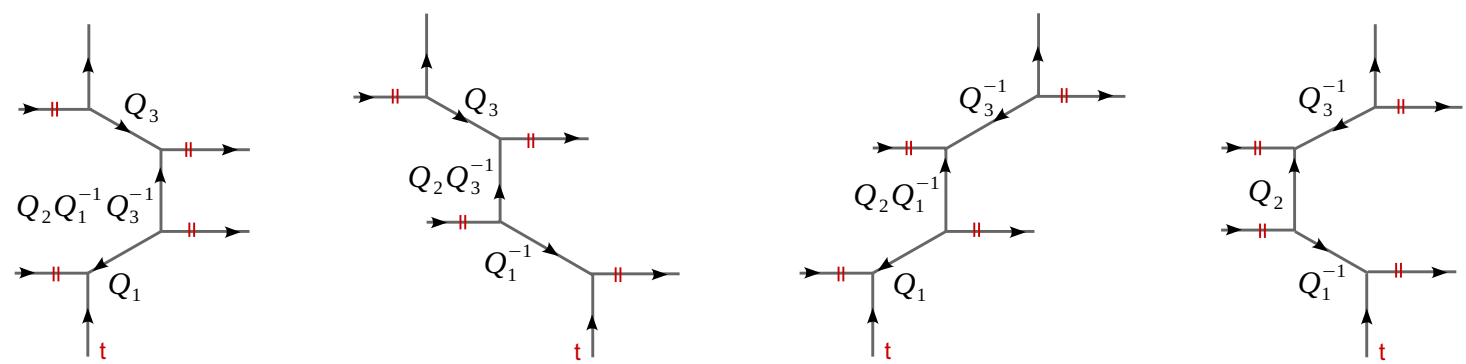

Figure 10. Different strip diagrams related by flops.

At the level of the topological strings partition functions, when naively setting $Q_{i}=\tilde{Q}_{i}$, for all $i=1,2,3$, the expressions $\mathcal{Z}_{2}^{\text {top }}(6.4)$ and $\mathcal{Z}_{\text {strip }}^{\text {top }}(6.3)$ are identified with the partition function of $\mathrm{SU}(2) \times \mathrm{SU}(2)$ free $5 \mathrm{D}$ hypermultiplets on $\mathbb{R}^{4} \times \mathbb{S}^{1}$

$$
\begin{aligned}
\mathcal{Z}_{\text {hypers }}^{\mathbb{R}^{4} \times \mathbb{S}^{1}} & =\frac{\mathcal{Z}_{T_{2}}^{\text {top }}\left(Q_{1}, Q_{2}, Q_{3} ; t, q\right)}{\mathcal{M}\left(Q_{1} Q_{2}\right) \mathcal{M}\left(Q_{2} Q_{3}\right) \mathcal{M}\left(Q_{1} Q_{3} \frac{t}{q}\right)}=\frac{\mathcal{Z}_{\text {strip }}^{\text {top }}\left(Q_{1}, Q_{2}, Q_{3} ; t, q\right)}{\mathcal{M}\left(Q_{1} Q_{2}\right) \mathcal{M}\left(Q_{2} Q_{3} \frac{t}{q}\right)} \\
& =\frac{1}{\mathcal{M}\left(Q_{1} \sqrt{\frac{t}{q}}\right) \mathcal{M}\left(Q_{2} \sqrt{\frac{t}{q}}\right) \mathcal{M}\left(Q_{3} \sqrt{\frac{t}{q}}\right) \mathcal{M}\left(Q_{1} Q_{2} Q_{3} \sqrt{\frac{t}{q}}\right)}
\end{aligned}
$$

up to products of $\mathcal{M}$ functions which are interpreted as the non-full spin content [30]. The topological string partition function contains extra degrees of freedom from strings stretching between the external parallel legs which do not transform properly under the 5D Lorenz group and thus have to be removed in order to obtain the 5D partition function [30, 32].

Below we give a systematic presentation comparing the $T_{2}$ and strip geometries, taking into account the various possible ways to flop internal edges of the web diagrams for each of these.

\subsection{Comparison}

We now want to compare the $T_{2}$ partition functions (3.28) and (3.31) with the result for the strip vertex. The computations can be found in [41, 44, 45, 59], and are reviewed in [54]. The result for the diagrams on the left and on the right of figure 10 is found to be

$$
Z_{l}^{\text {strip }}=\frac{\mathcal{M}\left(Q_{2}\right) \mathcal{M}\left(Q_{2}\left(Q_{1} Q_{3}\right)^{-1}\right)}{\prod_{i=1,3} \mathcal{M}\left(Q_{i}\right) \mathcal{M}\left(Q_{2} Q_{i}^{-1}\right)}, \quad Z_{r}^{\text {strip }}=\frac{\mathcal{M}\left(Q_{2}\right) \mathcal{M}\left(Q_{2}\left(Q_{1} Q_{3}\right)^{-1}\right)}{\prod_{i=1,3} \mathcal{M}\left(Q_{i}^{-1}\right) \mathcal{M}\left(Q_{2} Q_{i}^{-1}\right)} .
$$

Let us start with the leftmost diagram in figure 10. We may parameterise

$$
Q_{1}=q^{\beta\left(p_{3}-p_{1}-p_{2}\right)}, \quad Q_{3}=q^{\beta\left(p_{3}+p_{2}-p_{1}\right)}, \quad Q_{2}=q^{2 \beta p_{3}},
$$

where $p_{i}, i=1,2,3$, are the distances between the pairs of parallel lines containing the external edges of the strip diagram depicted in figure 11. Similar parameterisations can be introduced for the remaining cases. The resulting formulae for the limit $q \rightarrow 1$ can be written in the form

$$
\mathcal{Z}_{l}^{\text {strip }}=\frac{[\text { Leg factors }]}{\prod_{\epsilon, \epsilon^{\prime}} G_{\beta}\left(p_{3}+\epsilon p_{1}+\epsilon^{\prime} p_{2}\right)}, \quad \mathcal{Z}_{\mathrm{r}}^{\text {strip }}=\frac{[\text { Leg factors }]}{\prod_{\epsilon, \epsilon^{\prime}} G_{\beta}\left(p_{1}+\epsilon p_{3}+\epsilon^{\prime} p_{2}\right)} .
$$




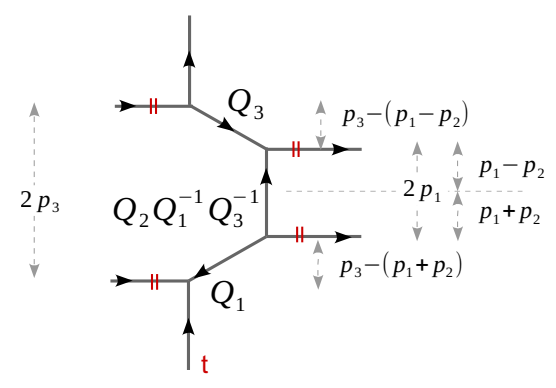

Figure 11. Parametrisation of distances between pairs of external parallel legs of a strip.

The result for the second strip diagram takes the form

$$
\mathcal{Z}_{m}^{\text {strip }}=\frac{[\text { Leg factors }]}{G_{\beta}\left(p_{1}+p_{2}+p_{3}\right) \prod_{i=1}^{3} G_{\beta}\left(p_{1}+p_{2}+p_{3}-2 p_{i}\right)},
$$

while for the third diagram, $\mathcal{Z}_{m_{2}}^{\text {strip }}$ differs from this by the replacement $p_{2} \rightarrow-p_{2}$.

Let us now compare these results to the ones found for $T_{2}$. First one may note that chamber $\mathfrak{P}_{\mathbb{R}}^{(2)}$ is not covered by the strip diagrams. One may otherwise observe the following relations

$$
\mathcal{Z}^{\mathrm{T}_{2}^{(3)}} \simeq \mathcal{Z}_{l}^{\text {strip }}, \quad \mathcal{Z}^{\mathrm{T}_{2}^{(\mathrm{s})}} \simeq \mathcal{Z}_{m}^{\text {strip }}, \quad \mathcal{Z}^{\mathrm{T}_{2}^{(1)}} \simeq \mathcal{Z}_{r}^{\text {strip }}
$$

where $\simeq$ means equality up to leg factors. We see that the results calculated using $T_{2}$ vertex and strip agree within each chamber up to leg factors.

\section{$7 \quad$ B-model picture}

We'd now like to shed some light on our findings using the tools offered by the B-model approach to topological string theory.

\subsection{Relation between mirror curves of strip and $T_{2}$}

In the above we had observed simple relations between $T_{2}$ vertices and strips. This is not surprising in view of the fact that the toric CY described by the $T_{2}$ vertex and the strip have mirror manifolds related by a coordinate change.

Indeed, the mirror of the $T_{2}$ toric diagram is known [30] to be the curve in $\mathbb{C}^{*} \times \mathbb{C}^{*}$ defined by the equation

$$
W^{2}-\left(P_{2}+P_{2}^{-1}\right) W+\left(P_{3}+P_{3}^{-1}\right) W T-\left(P_{1}+P_{1}^{-1}\right) T+T^{2}+1=0 .
$$

It is easy to check that the external legs of the $T_{2}$ toric diagram describe the infinite ends of the curve (7.1). Changing coordinates $T=\mathrm{U}\left(W-P_{2}\right)$ one gets

$$
W\left(U+P_{3}\right)\left(U+P_{3}^{-1}\right)=P_{2}\left(U+P_{1} P_{2}^{-1}\right)\left(U+P_{1}^{-1} P_{2}^{-1}\right) .
$$

This is recognised as the mirror of the toric diagram for the strip. A dual interpretation of this relation in terms of the intersecting brane systems one is provided by the two successive Hanany-Witten moves depicted in figure 12. 

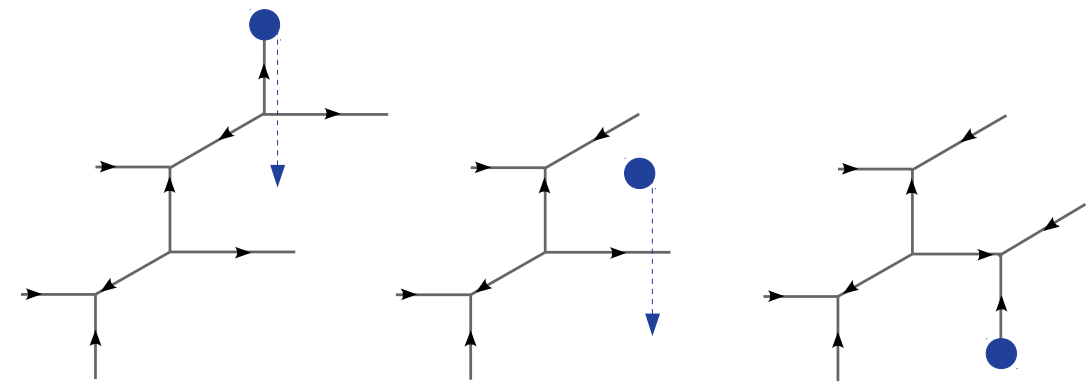

Figure 12. Starting with the strip geometry, the $T_{2}$ geometry can be obtained via two successive Hanany-Witten moves. The blue dot represents the 7-brane on which the 5-branes end.

The fact that the local CY mirror to the toric geometries associated to the strip and $T_{2}$ diagrams are related by a change of coordinates suggests that the corresponding topological string partition functions should be related. This gives us a simple way to predict relations between the partition functions $\mathcal{Z}^{T_{2}}$ and $\mathcal{Z}^{\text {strip }}$, as observed in section 6 above. To make this argument precise one needs to have a more direct way to compute the topological string partition functions within the B-model description. This is provided by the matrix model representation predicted by the Gopakumar-Vafa geometric transitions, as will be discussed next.

\subsection{Geometric transitions and matrix models}

It is well-known that the matrix model representation of topological string partition function reflects a duality between closed and open topological string referred to as geometric transition $[23,60]$. We will briefly discuss the implications of these dualities for the case of our interest. This can be done directly on the level of the four-dimensional partition functions obtained in the limit $q \rightarrow 1$ we are mostly interested in.

In the B-model one considers the local CY defined by the equation

$$
x^{2}(x-1)^{2} y^{2}=p_{3}^{2} x^{2}-\left(p_{3}^{2}+p_{1}^{2}-p_{2}^{2}\right) x+p_{1}^{2},
$$

which can be written as

$$
\left(y-\frac{p_{1}}{x}-\frac{p_{2}}{x-1}\right)\left(y+\frac{p_{1}}{x}+\frac{p_{2}}{x-1}\right)=\frac{\left(p_{3}-p_{1}-p_{2}\right)\left(p_{3}+p_{2}+p_{1}\right)}{x(x-1)} .
$$

Defining

$$
W(x)=p_{1} \log (x)+p_{2} \log (x-1),
$$

we may write equation (7.4) in the form

$$
y^{2}-\left(W^{\prime}(x)\right)^{2}=\frac{p_{3}^{2}-\left(p_{1}+p_{2}\right)^{2}}{x(x-1)} .
$$

In this form we may recognise the curve (7.3) as a resolution of the singular curve $y^{2}-$ $\left(W^{\prime}(x)\right)^{2}=0$. 
For integer values of $s=p_{3}-p_{2}-p_{1}$ one expects to have a dual description of the closed topological string in terms of an open topological string on a deformation of the singular curves obtained by setting $p_{3}=p_{2}+p_{1}$ and wrapping $s$ D-branes on the resulting $S^{3}$. The arguments of $[23,60]$ then lead to the prediction that

$$
\left.\mathcal{Z}^{\mathrm{cl}}\left(p_{1}, p_{2}, p_{3}\right)\right|_{p_{3}-p_{2}-p_{1}=s}=\mathcal{Z}^{\mathrm{op}}\left(p_{1}, p_{2} ; s\right)
$$

with $\mathcal{Z}^{\mathrm{op}}\left(p_{1}, p_{2} ; s\right)$ having a representation as a multiple integral

$$
\mathcal{Z}^{\mathrm{op}}\left(p_{1}, p_{2} ; s\right)=\int_{\mathcal{C}_{s}} d x_{1} \ldots d x_{s} \prod_{k<l}\left(x_{k}-x_{l}\right)^{2} \prod_{k=1}^{s} e^{\frac{1}{\lambda} W\left(x_{k}\right)},
$$

for a certain choice of the contour $\mathcal{C}_{s}$. The results for $\mathcal{Z}^{\mathrm{op}}\left(p_{1}, p_{2} ; s\right)$ will of course depend sensitively on $\mathcal{C}_{s}$. As stressed in [24] one should consider the choice of $\mathcal{C}_{s}$ as an important part of the non-perturbative definition of topological string theory. A natural candidate for the contour $\mathcal{C}_{s}$ has been identified in [24]. It amounts to defining $\mathcal{Z}^{\mathrm{op}}\left(p_{1}, p_{2} ; s\right)$ as the Selberg integral (5.17).

We may now observe that this proposal is perfectly consistent with the results of the topological vertex computations. The comparison is possible in the chamber of the parameter space where the real part of $p_{3}-p_{2}-p_{1}$ is positive. In this chamber one may use the toric diagram depicted in figure 11. In section 6 we had already observed that $\mathcal{Z}_{l}^{\text {strip }}$ is given by the Selberg integral, in perfect agreement with the prediction (7.7).

In the cases where $p_{3}<p_{2}+p_{1}$ one can no longer apply this reasoning. However, there is a second possibility for representing the curve (7.3) in the form (7.6), given by the formula

$$
\left(y-\frac{p_{1}}{x}+\frac{p_{2}}{x-1}\right)\left(y+\frac{p_{1}}{x}-\frac{p_{2}}{x-1}\right)=\frac{\left(p_{3}-p_{1}+p_{2}\right)\left(p_{3}-p_{2}+p_{1}\right)}{x(x-1)} .
$$

The same arguments as had been used to arrive at (7.7) can now be applied, for example in the case that $p_{3}-p_{1}+p_{2}=s$, with $s$ being a positive integer, leading to the prediction that

$$
\left.\mathcal{Z}^{\mathrm{cl}}\left(p_{1}, p_{2}, p_{3}\right)\right|_{p_{3}-p_{1}+p_{2}=s}=\tilde{\mathcal{Z}}^{\mathrm{op}}\left(p_{1}, p_{2} ; s\right)
$$

with $\mathcal{Z}^{\mathrm{op}}\left(p_{1}, p_{2} ; s\right)$ having a representation as a multiple integral

$$
\tilde{\mathcal{Z}}^{\mathrm{op}}\left(p_{1}, p_{2} ; s\right)=\int_{\tilde{\mathcal{C}}_{s}} d x_{1} \ldots d x_{s} \prod_{k<l}\left(x_{k}-x_{l}\right)^{2} \prod_{k=1}^{s} e^{\frac{1}{\lambda} \tilde{W}\left(x_{k}\right)},
$$

for a certain choice of the contour $\tilde{\mathcal{C}}_{s}$, and

$$
\tilde{W}(x)=p_{1} \log (x)-p_{2} \log (x-1) .
$$

One should observe, however, that the contour $\mathcal{C}_{s}$ proposed in [24] is not suitable for the second case, as it would yield a divergent integral, in general. At the upper limit of integration one encounters a singularity of the form $(1-x)^{-p_{2}}$ with $p_{2}$ positive. In this 
case it is natural to replace the contour by a half-open multi-contour $\mathcal{C}_{s}^{\prime}$ starting at $x_{k}=0$, encircling $x_{k}=1$, and returning to $x_{k}$ for $k=1, \ldots, s$, assuming that the contour of integration for $x_{k}$ is in the interior of the disc surrounded by the contour for $x_{l}$ if $k<l$. By deforming the contours ${ }^{6}$ it is possible to show that

$$
\tilde{\mathcal{Z}}^{\mathrm{op}}\left(p_{1}, p_{2} ; s\right)=\mathcal{Z}^{\mathrm{op}}\left(p_{1},-p_{2} ; s\right) \prod_{k=0}^{s-1}(-2 \mathrm{i}) \sin \left(\pi \beta\left(-2 p_{2}+k \beta\right)\right) .
$$

This prediction can be compared with the results we had obtained above. It can be applied, on the one hand, to the case of the strip in the case where $p_{2}>0$ and $p_{1}-p_{2}<p_{3}<p_{2}+p_{1}$, represented by second diagram from the left in figure 10. It can, on the other hand, be applied in the case of the $T_{2}$ vertex. We find that our previous results are perfectly consistent with (7.10). It is interesting to observe that the factor relating the partition functions associated to the two chambers is directly related to the monodromy factors reflecting the change of the contours.

Reconsidering the identification between the Liouville parameters $a_{1}, a_{2}, a_{3}$ and the geometric parameters $p_{1}, p_{2}, p_{3}$ it is important to note that the partition functions associated to the toric diagrams on the left half of figure 11 are related to the counterparts on the right half found by a reflection along an axis in the middle by $p_{2} \rightarrow-p_{2}$. Consideration of the strip diagrams can therefore fix the relation between $a_{2}$ and $p_{2}$ only up to a sign. The situation is better in the case of the $T_{2}$ diagram. Formula (7.9) suggests to identify

$$
\tilde{W}(x)=p_{1} \log (x)-p_{2} \log (x-1)=a_{1} \log (x)+a_{2} \log (x-1),
$$

leading to the relation $p_{2}=-a_{2}$ already adopted in the above.

\subsection{Topological recursion}

Another technique for the reconstruction of the topological string partition function from the curve $\Sigma$ is the topological recursion [61,62]. This method has recently been applied to the case at hand in $[63,64]$. The result can be represented in the form

$$
\left[\log \mathcal{Z}\left(p_{3}, p_{2}, p_{1}\right)\right]_{\mathrm{f}}=\left[\log \frac{\prod_{\epsilon, \epsilon^{\prime}= \pm} G\left(1+p_{3}+\epsilon p_{2}+\epsilon^{\prime} p_{1}\right)}{G\left(1+2 p_{3}\right) G\left(1+2 p_{2}\right) G\left(1+2 p_{1}\right) G(1)}\right]_{\mathrm{f}},
$$

where $p_{i}=\frac{1}{\lambda} \nu_{i}$ and $[\log G(1+w)]_{\mathrm{f}}$ is the following formal power series

$$
[\log G(1+w)]_{\mathrm{f}}=\frac{1}{2}\left(w^{2}-\frac{1}{6}\right) \log (w)-\frac{3}{4} w^{2}-w \zeta^{\prime}(0)+\zeta^{\prime}(-1)-\sum_{g=2} \frac{B_{2 g}}{2 g(2 g-2)} w^{2-2 g} .
$$

\footnotetext{
${ }^{6}$ One may first deform the innermost contour in the sum of two segments infinitesimally below and above the real axis plus a small circle around 1 . The integral is thereby seen to be proportional to $2 \mathrm{i} \sin \left(2 \pi \beta p_{2}\right)$ times an integral having the interval $[0,1]$ as the contour of integration over $x_{1}$. The contour of integration for $x_{2}$ may then be deformed in a similar way, and so on. We will assume that the definition of the contour $\mathcal{C}_{s}^{\prime}$ involves an ordering ensuring that the result of the contour deformation above is an integral with integration variables $x_{1}, \ldots, x_{s}$ ordered along the interval $[0,1]$ as $0<x_{s}<x_{s-1}<\cdots<x_{1}<1$.
} 
It is known that this formal series represents the aymptotic expansion of the Barnes $G$ function (see e.g. [65]).

The formal series on the right side of (7.16) is Borel summable, as follows from the Binet type integral representation for the function $G(x)[65]$

$$
\begin{aligned}
\log G(1+w)=w \log \Gamma(w) & +\frac{w^{2}}{4}-\frac{B_{2}(w)}{2} \log w-\log A \\
& -\int_{0}^{\infty} \frac{d t}{t^{2}} e^{-t w}\left(\frac{1}{1-e^{-t}}-\frac{1}{t}-\frac{1}{2}-\frac{t}{12}\right) .
\end{aligned}
$$

Indeed, the integral on the right of equation (7.17) represents the Laplace transform of a function which is easily found to be the Borel-transform of the asymptotic series in (7.16).

However, the Borel summation displays a Stokes phenomenon. The result depends on the chamber in the parameter space one is in. The integral in (7.17) converges for $\operatorname{Re}(w)>0$. When $\operatorname{Re}(w)<0$ one may notice that $G(1-w)$ has almost the same asymptotic expansion as $G(1+w)$ has. There are two differences, though. The first is due to the factor $\log (-w)$, the second to the term proportional to $\zeta^{\prime}(0)$. There is, of course, an inevitable ambiguity in the choice of the branch of the logarithm in (7.16). Replacing $\log (w)$ by $\log (-w)$ appears to be a natural way to fix this ambiguity. This motivates us to define the piecewise holomorphic function $\widehat{G}(w)$ by the equation

$$
\log \widehat{G}(1+w)= \begin{cases}\log G(1+w) & \text { for } \operatorname{Re}(w)>0, \\ \log G(1-w)-2 w \zeta^{\prime}(0) & \text { for } \operatorname{Re}(w)<0,\end{cases}
$$

With this definition one may represent the result in the form

$$
\log \mathcal{Z}\left(p_{3}, p_{2}, p_{1}\right)=\log \frac{\prod_{\epsilon, \epsilon^{\prime}= \pm} \widehat{G}\left(1+p_{3}+\epsilon p_{2}+\epsilon^{\prime} p_{1}\right)}{\widehat{G}\left(1+2 p_{3}\right) \widehat{G}\left(1+2 p_{2}\right) \widehat{G}\left(1+2 p_{1}\right) G(1)},
$$

assuming that all variables $p_{i}$ are real and positive. Noting that the term proportional to $\zeta^{\prime}(0)$ only affects the leg factors one recognises the same form as was found in section 3.4.

Let us note that the partition functions represent analytic functions within their respective domains of definition. Requiring that this is the case fixes the signs in front of the combination $p_{3}+\epsilon p_{2}+\epsilon^{\prime} p_{1}$ appearing in the arguments of the Barnes functions. ${ }^{7}$ This observation is naturally explained by the observations above concerning the Borel summation of the topological recursion.

\section{$8 \quad T_{N}$ versus conformal blocks}

We are now going to formulate more precisely what an AGT-type correspondence between the $T_{N}$ theories and Toda CFT conformal blocks would mean. It should relate a) the partition functions of $T_{N}$ theories computed using the topological vertex, and b) conformal blocks of $A_{N-1}$ conformal Toda field theories. To this aim we will again compare with the case of the strip for which the relation to the AGT-correspondence is fairly well-understood.

\footnotetext{
${ }^{7}$ Note here that the Barnes G-function $G(1+x)$ is analytic if the real part of $x$ is positive, but has poles on the negative real axis.
} 
The basic observation is the following: Let $\mathcal{Z}^{\text {strip }}\left(\vec{\mu}, \vec{\nu} ; \mathbf{p}, \mathbf{p}^{\prime}, w ; q, t\right)$ be the partition function defined by the strip diagram with horizontal legs decorated by $N$-tuples of Young diagrams $\vec{\mu}=\left(\mu_{1}, \ldots, \mu_{N}\right), \vec{\nu}=\left(\nu_{1}, \ldots, \nu_{N}\right)$ (see for example figure 9 for the case $N=2$ ). The variables $\mathbf{p}$ and $\mathbf{p}^{\prime}$ are $N$-tuples of complex numbers assigned to the two sets of parallel external horizontal lines appearing on the left and on the right of the strip diagram, respectively. The variable $w$ is associated to the horizontal shift between the vertical lines emanating at the top and on the bottom of the strip diagram. The Kähler parameters associated to the internal edges can be easily expressed in terms of the variables $\mathbf{p}, \mathbf{p}^{\prime}$ and $w$. The parameters $q=e^{-R \epsilon_{1}}$ and $t=e^{R \epsilon_{2}}$ can be expressed in terms of the topological string coupling $\lambda^{2}=\epsilon_{1} \epsilon_{2}$ and the additional refinement parameter $\epsilon_{1}+\epsilon_{2}$ introduced in [21].

One may then consider the function $\mathcal{Z}^{\text {bif }}$ defined by the limit

$$
\mathcal{Z}_{\vec{\mu}, \vec{\nu}}^{\text {bif }}\left(\mathbf{p}, \mathbf{p}^{\prime}, w ; b\right):=\lim _{q \rightarrow 1} \frac{\mathcal{Z}_{\vec{\mu}, \vec{\nu}}^{\text {strip }}\left(\mathbf{p}, \mathbf{p}^{\prime}, w ; q, t\right)}{\mathcal{Z}_{\vec{\emptyset}, \vec{\emptyset}}^{\text {strip }}\left(\mathbf{p}, \mathbf{p}^{\prime}, w ; q, t\right)} .
$$

The function $\mathcal{Z}^{\text {bif }}$ is on the one hand known to be an important building block of the instanton partition functions of linear quiver theories, and it was on the other hand shown to be the matrix representing a particular type of intertwining operator between represen-

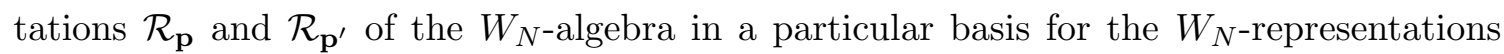
called AFLT-basis after Alba-Fateev-Litvinov-Tarnopolsky [2, 12],

$$
\mathcal{Z}_{\vec{\mu}, \vec{\nu}}^{\text {bif }}\left(\mathbf{p}, \mathbf{p}^{\prime}, w ; b\right)={ }_{\mathbf{p}^{\prime} ; b}\left\langle\vec{\nu}\left|\mathrm{V}_{\mathbf{p}^{\prime}, \mathbf{p}}(w)\right| \vec{\mu}\right\rangle_{\mathbf{p} ; b} .
$$

The AFLT basis diagonalises a natural abelian sub-algebra of the product of the $W_{N}$ algebra with a free boson algebra within a Fock-space representation of this algebra. Identity (8.2) is the main ingredient in the proof of the AGT correspondence given in $[2,12]$.

One may, of course, simply define an operator $\mathcal{V}_{\mathbf{p}^{\prime}, \mathbf{p}}(w)$ on a vector space $\mathcal{F}$ having a basis formed by vectors $|\vec{\mu}\rangle$ labelled by $N$-tuples of Young diagrams $\vec{\mu}$ such that

$$
\mathcal{Z}_{\vec{\mu}, \vec{\nu}}^{\operatorname{strip}}\left(\mathbf{p}, \mathbf{p}^{\prime}, w ; q, t\right)=\left\langle\vec{\nu}\left|\mathcal{V}_{\mathbf{p}^{\prime}, \mathbf{p}}(w)\right| \vec{\mu}\right\rangle .
$$

The following results from $[66,67]$ and references therein clarify in which sense the operator

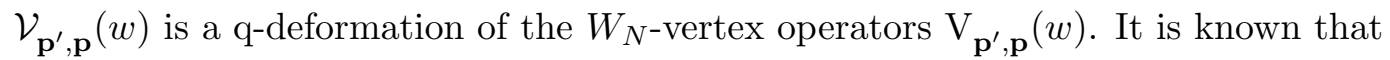

- The spaces $\mathcal{F}_{\mathbf{p}}$ are modules of a Hopf algebra called Ding-Iohara-Miki-algebra (DIM algebra). There exists a Fock-space realisation $\mathcal{F}_{\mathbf{p}}$ of the DIM algebra in which the states $|\vec{\mu}\rangle$ can be realised in terms of generalised Macdonald functions, $|\vec{\mu}\rangle \equiv$ $|\vec{\mu}\rangle_{\mathbf{p} ; q, t}$. The basis $|\vec{\mu}\rangle$ diagonalises a large abelian sub-algebra in the DIM algebra with eigenvalues being functions of an $\mathrm{N}$-tuple of parameters $\mathbf{p}=\left(p_{1}, \ldots, p_{N}\right)$.

- The q-deformation $q-W_{N}$ of the W-algebra $W_{N}$ can be embedded into the DIM algebra, making the spaces $\mathcal{F}_{\mathbf{p}}$ highest weight representations of the $q$ - $W_{N}$-algebra. The eigenvalues of the zero modes of the $q$ - $W_{N}$-algebra determining the $q$ - $W_{N}$-module can be expressed in terms of the parameters $\mathbf{p}$. For $q \rightarrow 1$ one finds that

$$
\lim _{R \rightarrow 0}|\vec{\mu}\rangle_{\mathbf{p} ; q, t}=|\vec{\mu}\rangle_{\mathbf{p} ; b}
$$

where the vectors $|\vec{\mu}\rangle_{\mathbf{p} ; b}$ form the AFLT-basis. 
- The operators $\mathcal{V}_{\mathbf{p}, \mathbf{p}^{\prime}}(w)$ defined from $\mathcal{Z}^{\text {strip }}$ in this way are intertwining operators between representations $\mathcal{F}_{\mathbf{p}}$ and $\mathcal{F}_{\mathbf{p}^{\prime}}$ of the DIM algebra, characterised by simple commutation relations with the generators of the DIM algebra [66] or with the generators of the $q$ - $W_{N}$-algebra [67].

These observations explain how the symmetry algebra $W_{N}$ in the AGT correspondence can be understood as a limit of similar structures of topological string theory. They explain, in particular, why the partition functions associated to toric diagrams obtained by gluing two strip diagrams get related to the conformal blocks associated to spheres with four punctures in the limit $q \rightarrow 1$. Such conformal blocks can be represented as matrix elements of compositions of the vertex operators $\mathrm{V}_{\mathbf{p}^{\prime}, \mathbf{p}}(w)$, like, for example

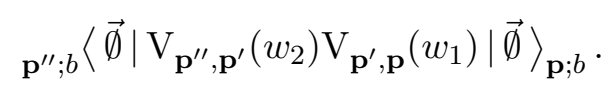

Inserting a complete set of states from the representation $\mathcal{F}_{\mathbf{p}^{\prime}}$ between the vertex operators $\mathrm{V}_{\mathbf{p}^{\prime \prime}, \mathbf{p}^{\prime}}\left(w_{2}\right)$ and $\mathrm{V}_{\mathbf{p}^{\prime}, \mathbf{p}}\left(w_{1}\right)$ yields the familiar power series expansions of the conformal blocks with coefficients given by products of the matrix elements related to $\mathcal{Z}^{\text {bif }}$ via (8.2).

If a generalisation of the AGT-correspondence holds for all class $\mathcal{S}$ theories, as expected, it would imply relations between the partition functions of the $T_{N}$ theories and $W_{N}$ conformal blocks that we'll now formulate a bit more precisely. The diagrams representing the toric CY used to engineer the $T_{N}$ theories within string theory now have three very similar legs consisting of $N$ parallel lines. Associating $N$-tuples of partitions to each of the three legs allows one to define partition functions

$$
\mathcal{Z}_{\vec{\nu}_{1}, \vec{\nu}_{2}, \vec{\nu}_{3}}^{T_{N}}\left(\mathbf{p}_{1}, \mathbf{p}_{2}, \mathbf{p}_{3} ; \mathbf{a} ; t, q\right)
$$

depending on tuples of partitions $\vec{\nu}_{1}, \vec{\nu}_{2}, \vec{\nu}_{3}$ associated to the three legs. The Kähler parameters can be naturally parameterised through three $N$-tuples of parameters $\mathbf{p}_{1}, \mathbf{p}_{2}$, $\mathbf{p}_{3}$ describing the asymptotic geometry of the legs, supplemented by $d=\frac{1}{2}(N-2)(N-1)$ parameters a parameterising the widths of the internal faces in the toric diagram.

It is tempting to define vertex operators from $\mathcal{Z}^{T_{N}}$ by a construction similar to the one outlined above for the case of the strip. A natural analog of (8.3) could be, for example,

$$
\mathcal{Z}_{\vec{\mu}, \vec{\emptyset}, \vec{\nu}}^{T_{N}}\left(\mathbf{p}_{1}, \mathbf{p}_{2}, \mathbf{p}_{3}, \mathbf{a} ; q, t\right)=\left\langle\vec{\nu}\left|\mathcal{V}_{\mathbf{p}_{3}, \mathbf{p}_{1}}^{\mathbf{p}_{2}, \mathbf{a}}(1)\right| \vec{\mu}\right\rangle .
$$

It is not clear at the moment if the vertex operator $\mathcal{V}_{\mathbf{p}_{3}, \mathbf{p}_{1}}^{\mathbf{p}_{2}, \mathbf{a}}$ (1) defined through (8.7) has a limit for $q \rightarrow 1$ which can be identified with a vertex operator $\mathrm{V}_{\mathbf{p}_{3}, \mathbf{p}_{1}}^{\mathbf{p}_{2}, \mathbf{a}}(1)=\left.\mathrm{V}_{\mathbf{p}_{3}, \mathbf{p}_{1}}^{\mathbf{p}_{2}, \mathbf{a}}(w)\right|_{w=1}$ intertwining between representations of the $W_{N}$-algebra. This does not seem to be known even for $N=2$, where one might expect to recover the familiar vertex operators of the Virasoro algebra in this way. The matrix elements of such vertex operators are determined by simple commutation relations with the generators of the Virasoro algebra. It is not clear if the limit $q \rightarrow 1$ of the objects in (8.7) will reproduce these matrix elements. A necessary condition for this to be the case would be a relation between matrix elements of compositions of the vertex operators $\mathrm{V}_{\mathbf{p}_{3}, \mathbf{p}_{1}}^{\mathbf{p}_{2}}(w)$ similar to (8.5) and the limit $q \rightarrow 1$ of glued $T_{N}$-vertices. We will check this condition below. 
For $N>2$ one defines a family of vertex operators through (8.7) having $d$ parameters a. This is indeed as expected. For generic triples of representations $\mathcal{R}_{\mathbf{p}_{1}}, \mathcal{R}_{\mathbf{p}_{2}}, \mathcal{R}_{\mathbf{p}_{3}}$ one expects to find an infinite dimensional vector space of chiral vertex operators which has a basis spanned by chiral vertex operators $\mathrm{W}_{\mathbf{p}_{3}, \mathbf{p}_{1}}^{\mathbf{p}_{2}, \mathbf{s}}(w)$ labelled by $d$ parameters $\mathbf{s}$, see $[68,69]$ for discussions of this issue. It is not clear at the moment if the operators $\mathcal{V}_{\mathbf{p}_{3}, \mathbf{p}_{1}}^{\mathbf{p}_{2}, \mathbf{a}}(1)$ defined through (8.7) have a limit $q \rightarrow 1$ defining operators $\mathrm{V}_{\mathbf{p}_{3}, \mathbf{p}_{1}}^{\mathbf{p}_{2}, \mathbf{a}}(w)$ forming a basis for the space of chiral vertex operators. Even if this was the case it is not clear if such a basis would have a more direct description in conformal field theory. The vertex operators $V_{\mathbf{p}_{3}, \mathbf{p}_{1}}^{\mathbf{p}_{2}, \mathbf{a}}(w)$ might, for example, turn out to be linear combinations of vertex operators $\mathrm{W}_{\mathbf{p}_{3}, \mathbf{p}_{1}}^{\mathbf{p}_{2}, \mathbf{s}}(w)$ constructed with the help of the free field representation of the $W_{N}$-algbra in [69].

These issues simplify considerably when $N=2$, but do not become trivial. Even if the space of chiral vertex operators is generically one-dimensional in this case, there still is non-trivial information needed to fix a basis for this one-dimensional vector space. This information is provided by the normalisation factor

$$
N\left(\mathbf{p}_{3}, \mathbf{p}_{2}, \mathbf{p}_{1}\right):={ }_{\mathbf{p}_{3}, b}\left\langle\vec{\emptyset}\left|\mathrm{V}_{\mathbf{p}_{3}, \mathbf{p}_{1}}^{\mathbf{p}_{2}}(1)\right| \vec{\emptyset}\right\rangle_{\mathbf{p}_{1}, b} .
$$

The results obtained in the previous section determine a preferred choice of this normalisation factor for each region in the parameter space described in section 3.4. The relation between topological string partition functions and three-point conformal blocks obtained in this way can be seen as a variant of the AGT-correspondence.

\section{$9 \quad$ Gluing $T_{2}$ building blocks vs. strips}

With the aim to better understand if the $T_{2}$ vertex can be used as a building block, we will glue two $T_{2}$ vertices and compute the partition function of the resulting brane web using the topological vertex formalism, comparing this result to the gluing of strip blocks. The two geometries produced by the gluing process are depicted in figure 13 and they are both used to compute the partition function of the $5 \mathrm{D} \mathcal{N}=1, \mathrm{SU}(2)$ four-flavour theory on $\mathbb{R}_{q, t}^{4} \times \mathbb{S}^{1}$, up to what is referred to in the literature as the non-full spin content [30]. This represents the degrees of freedom coming from the external parallel legs. The webs depicted in figure 13 are related by two flops, applied to the edges of the octagon decorated by the Kähler parameters $Q_{m_{2}}$ and $Q_{m_{4}}$, or equivalently to the edges with Kähler parameters $Q_{3}$ and $Q_{3}^{\prime}$.

The partition function for the $\mathrm{SU}(2), N_{f}=4$ theory in $5 \mathrm{D}$, calculated with the refined topological vertex for the brane diagram on the left of figure 13, can be expressed in terms of the partition functions $\mathcal{Z}_{\text {strip }}$ of the strips glued to create the octagonal web

$$
\mathcal{Z}_{\text {oct }}\left(\mathbf{Q}_{\mathbf{m}}, \mathbf{Q}_{\mathbf{m}^{\prime}}, \mathbf{Q}_{B}^{\prime} ; t, q\right)=\sum_{\tau_{1}, \tau_{2}}\left(-Q_{B_{1}}^{\prime}\right)^{\left|\tau_{1}\right|}\left(-Q_{B_{2}}^{\prime}\right)^{\left|\tau_{2}\right|} \mathcal{Z}_{\tau_{1}, \tau_{2}}^{\text {strip }}\left(\mathbf{Q}_{\mathbf{m}} ; t, q\right) \mathcal{Z}_{\tau_{2}^{t}, \tau_{1}^{t}}^{\text {strip }}\left(\mathbf{Q}_{\mathbf{m}^{\prime}} ; q, t\right)
$$

The dependance on the Kähler parameter $Q_{F}$ is kept implicit in this equation. We will see below that, in order to recast this summation in the form of an instanton expansion where 

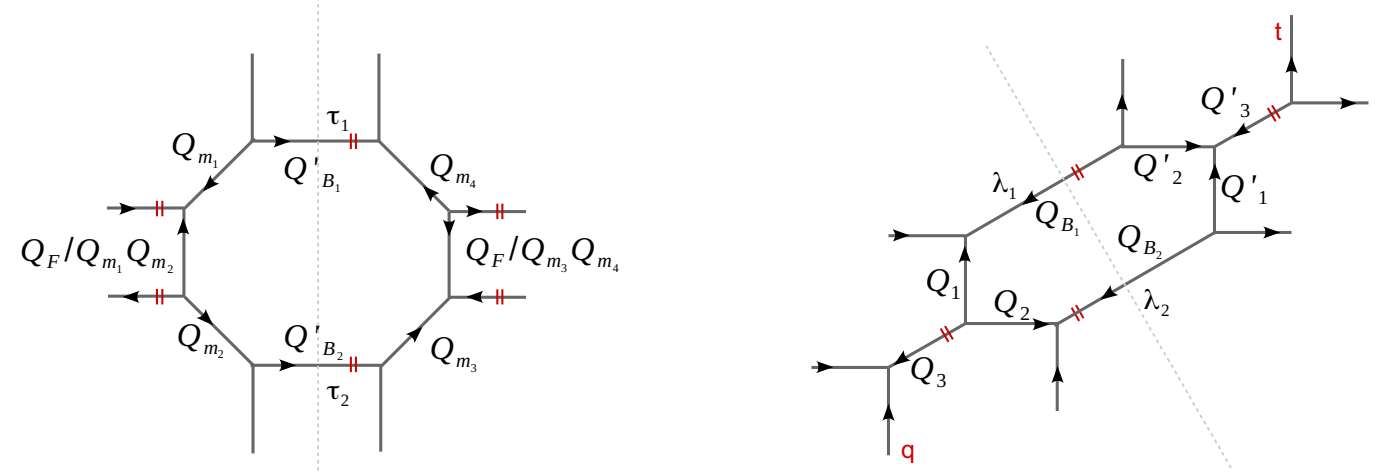

Figure 13. Brane diagrams corresponding to the $\mathcal{N}=1, \mathrm{SU}(2)$ four-flavour theory in $5 \mathrm{D}$, constructed by gluing strips (left) and two $T_{2}$ webs (right). Both the preferred direction and Kähler parameters are also indicated.

$\mathcal{Z}_{\text {oct }}=\mathcal{Z}_{\text {oct }}^{\text {pert }} \mathcal{Z}_{\text {oct }}^{\text {inst }}$, the Kähler parameters $\mathbf{Q}_{B}^{\prime}$ are first set to

$$
Q_{B_{1}}^{\prime}=u \frac{Q_{F}}{Q_{m_{1}} Q_{m_{4}}}, \quad Q_{B_{2}}^{\prime}=u \frac{Q_{F}}{Q_{m_{2}} Q_{m_{3}}},
$$

with $u$ the instanton counting parameter. It is possible to express two independent parameters $Q_{B_{i}}^{\prime}, i=1,2$, in terms of a single parameter $u$ because the Kähler parameters associated to the edges of a face of a brane diagram satisfy face constraints, here $Q_{m_{1}} Q_{B_{1}}^{\prime} Q_{m_{4}}=Q_{m_{2}} Q_{B_{2}}^{\prime} Q_{m_{3}}$. Equation (9.23) allows to represent the instanton part of the partition function in the form

$$
\mathcal{Z}_{\text {oct }}^{\text {inst }}\left(\mathbf{Q}_{\mathbf{m}}, \mathbf{Q}_{\mathbf{m}^{\prime}} ; t, q\right)=\sum_{k=0}^{\infty} u^{k} \mathcal{Z}_{k}^{\text {strip }}\left(\mathbf{Q}_{\mathbf{m}}, \mathbf{Q}_{\mathbf{m}^{\prime}} ; t, q\right),
$$

which is known to reproduce the expansion of Virasoro four point conformal blocks in powers of the cross-ratio [1]. We compare this result to the analogous expansion obtained when gluing $T_{2}$ building blocks

$$
\mathcal{Z}_{\text {hex }}\left(\mathbf{Q}, \mathbf{Q}^{\prime}, \mathbf{Q}_{B} ; t, q\right)=\sum_{\lambda_{1}, \lambda_{2}}\left(-Q_{B_{1}}\right)^{\left|\lambda_{1}\right|}\left(-Q_{B_{2}}\right)^{\left|\lambda_{2}\right|} \mathcal{Z}_{\lambda_{1}, \lambda_{2}}^{T_{2}}(\mathbf{Q} ; t, q) \mathcal{Z}_{\lambda_{2}^{t}, \lambda_{1}^{t}}^{T_{2}}\left(\mathbf{Q}^{\prime} ; q, t\right) .
$$

Setting here the Kähler parameters $\mathbf{Q}_{B}$ to be

$$
Q_{B_{1}}=u Q_{2}, \quad Q_{B_{2}}=u Q_{2}^{\prime}
$$

similarly to equation $(9.23)$, we find the instanton expansion from $\mathcal{Z}_{\text {hex }}=\mathcal{Z}_{\text {hex }}^{\text {pert }} \mathcal{Z}_{\text {hex }}^{\text {inst }}$ is

$$
\mathcal{Z}_{\text {hex }}^{\text {inst }}\left(\mathbf{Q}, \mathbf{Q}^{\prime} ; t, q\right)=\frac{\mathcal{Z}_{\text {oct }}^{\text {inst }}\left(\mathbf{Q}_{\mathbf{m}}, \mathbf{Q}_{\mathbf{m}^{\prime}} ; t, q\right)}{\mathcal{M}\left(Q_{B_{1}}^{\prime}\right) \mathcal{M}\left(Q_{B_{2}}^{\prime} \frac{t}{q}\right)},
$$

assuming a simple and natural dictionary between $\mathbf{Q}, \mathbf{Q}^{\prime}$ and $\mathbf{Q}_{\mathbf{m}}, \mathbf{Q}_{\mathbf{m}^{\prime}}$ and removing what is known as the non-full spin content $\mathcal{M}\left(Q_{B_{1}}^{\prime}\right) \mathcal{M}\left(Q_{B_{2}}^{\prime} \frac{t}{q}\right)$. The comparison between the partition functions obtained by gluing $T_{2}$ blocks and strip geometries can only be done order by order in the instanton expansion, unless one is able to perform the sum in equation (9.15) analytically and derive a product formula. It is a technically very difficult computation and thus we will present here a check up to second order in the instanton parameter. 


\section{1 $\mathrm{SU}(2)$ four-flavour $\mathcal{N}=1$ theory in 5D from gluing of strips}

It will help to start by reviewing the case of the strip first. We can explicitly compute the topological strings partition function for the octagon diagram in figure 13, which is obtained by gluing two strips, like in $[53,54]$

$$
\begin{gathered}
\mathcal{Z}_{\text {oct }}\left(\mathbf{Q}_{m}, Q_{F}, \mathbf{Q}_{B}^{\prime} ; t, q\right)=\frac{\mathcal{M}\left(\frac{Q_{F}}{Q_{m_{1} Q_{m_{2}}}}\right) \mathcal{M}\left(\frac{Q_{F}}{Q_{m_{3}} Q_{m_{4}}}\right) \mathcal{M}\left(Q_{F}\right) \mathcal{M}\left(Q_{F} \frac{t}{q}\right)}{\prod_{i=1}^{4} \mathcal{M}\left(Q_{m_{i}} \sqrt{\frac{t}{q}}\right) \mathcal{M}\left(\frac{Q_{F}}{Q_{m_{i}}} \sqrt{\frac{t}{q}}\right)} \\
\sum_{\tau_{1}, \tau_{2}}\left(Q_{B_{1}}^{\prime}\right)^{\left|\tau_{1}\right|}\left(Q_{B_{2}}^{\prime} \frac{t}{q}\right)^{\left|\tau_{2}\right|} q^{\left\|\mid \tau_{1}\right\|^{2}} t^{\left\|\mid \tau_{2}^{t}\right\|^{2}} \prod_{\ell=1}^{2} \tilde{Z}_{\tau_{\ell}}(t, q) \tilde{Z}_{\tau_{\ell}^{t}}(q, t) \\
\frac{\prod_{i=1,4} \mathcal{N}_{\tau_{1} \emptyset}\left(Q_{m_{i}} \sqrt{\frac{t}{q}}\right) \mathcal{N}_{\emptyset \tau_{2}}\left(\frac{Q_{F}}{Q_{m_{i}}} \sqrt{\frac{t}{q}}\right) \prod_{i=2,3} \mathcal{N}_{\emptyset \tau_{2}}\left(Q_{m_{j}} \sqrt{\frac{t}{q}}\right) \mathcal{N}_{\tau_{1} \emptyset}\left(\frac{Q_{F}}{Q_{m_{j}}} \sqrt{\frac{t}{q}}\right)}{\mathcal{N}_{\tau_{1} \tau_{2}}\left(Q_{F}\right) \mathcal{N}_{\tau_{1} \tau_{2}}\left(Q_{F} \frac{t}{q}\right)} .
\end{gathered}
$$

The base Kähler parameters of the octagon must be identified like in (9.2), which is the same as equation (4.88) of [70]. Only after this step we can identify the perturbative and instanton parts for this partition function

$$
\mathcal{Z}_{\text {oct }}=\mathcal{Z}_{\text {oct }}^{\text {pert }} \mathcal{Z}_{\text {oct }}^{\text {inst }} \quad \text { with } \quad \mathcal{Z}_{\text {oct }}^{\text {pert }}=\frac{\mathcal{M}\left(\frac{Q_{F}}{Q_{m_{1}} Q_{m_{2}}}\right) \mathcal{M}\left(\frac{Q_{F}}{Q_{m_{3}} Q_{m_{4}}}\right) \mathcal{M}\left(Q_{F}\right) \mathcal{M}\left(Q_{F} \frac{t}{q}\right)}{\prod_{i=1}^{4} \mathcal{M}\left(Q_{m_{i}} \sqrt{\frac{t}{q}}\right) \mathcal{M}\left(\frac{Q_{F}}{Q_{m_{i}}} \sqrt{\frac{t}{q}}\right)} .
$$

With this factorization, the instanton sum

$$
\mathcal{Z}_{\text {oct }}^{\text {inst }}=1+u \mathcal{Z}_{\text {oct }}^{1-\text { inst }}+u^{2} \mathcal{Z}_{\text {oct }}^{2-\text { inst }}+\ldots
$$

starts at zero order with unity, where $\tau_{1}=\tau_{2}=\emptyset$. At the next order, the coefficient $\mathcal{Z}_{\text {oct }}^{1-\text { inst }}$ is given by

$$
\begin{aligned}
& \mathcal{Z}_{\text {oct }}^{1-\text { inst }}=\frac{q Q_{F}}{(1-q)(1-t)}\left(\frac{1}{Q_{m_{1}} Q_{m_{4}}}+\frac{1}{Q_{m_{2}} Q_{m_{3}}} \frac{t}{q}\right) \\
& +\frac{q}{t} \frac{\left(1+\frac{q}{t}\right)\left(1+\sum_{\substack{i \neq j \\
i, j=1}}^{4} \frac{Q_{F}}{Q_{m_{i}} Q_{m_{j}}}+\frac{Q_{F}^{2}}{\prod_{k=1}^{4} Q_{m_{k}}}\right)-\sqrt{\frac{q}{t}}\left(1+Q_{F}\right) \sum_{i=1}^{4}\left(\frac{1}{Q_{m_{i}}}+\frac{Q_{F} Q_{m_{i}}}{\prod_{j=1}^{4} Q_{m_{j}}}\right)}{(1-q)\left(1-t^{-1}\right)\left(1-Q_{F} q / t\right)\left(1-Q_{F}^{-1} q / t\right)} .
\end{aligned}
$$

Removing from equation (9.7) what is referred to in literature as the non-full spin content [30], and which in this case is given by the product $\mathcal{M}\left(Q_{B_{1}}^{\prime}\right) \mathcal{M}\left(Q_{B_{2}}^{\prime} \frac{t}{q}\right)$, gives

$$
\mathcal{Z}_{\text {norm }}=\frac{\mathcal{Z}_{\text {oct }}\left(\mathbf{Q}_{m}, Q_{F}, \mathbf{Q}_{B}^{\prime} ; t, q\right)}{\mathcal{M}\left(Q_{B_{1}}^{\prime}\right) \mathcal{M}\left(Q_{B_{2}}^{\prime} \frac{t}{q}\right) \mathcal{M}\left(\frac{Q_{F}}{Q_{m_{1}} Q_{m_{2}}}\right) \mathcal{M}\left(\frac{Q_{F}}{Q_{m_{3}} Q_{m_{4}}}\right)} .
$$

This is the partition function of the $5 \mathrm{D} \mathcal{N}=1, \mathrm{SU}(2)$ four-flavour theory on $\mathbb{R}_{q, t}^{4} \times \mathbb{S}^{1}$ and the non-full spin content corresponds to (non 5D) degrees of freedom coming from the pairs of parallel external legs. The parameter identification (9.2) then allows to split the 
partition function into a product of perturbative and instanton parts, where

$$
\mathcal{Z}_{\text {norm }}=\mathcal{Z}_{\text {norm }}^{\text {pert }} \mathcal{Z}_{\text {norm }}^{\text {inst }} \quad \text { with } \quad \mathcal{Z}_{\text {norm }}^{\text {pert }}=\frac{\mathcal{M}\left(Q_{F}\right) \mathcal{M}\left(Q_{F} \frac{t}{q}\right)}{\prod_{i=1}^{4} \mathcal{M}\left(Q_{m_{i}} \sqrt{\frac{t}{q}}\right) \mathcal{M}\left(\frac{Q_{F}}{Q_{m_{i}}} \sqrt{\frac{t}{q}}\right)}
$$

and

$$
\mathcal{Z}_{\text {norm }}^{\text {inst }}\left(\mathbf{Q}_{m}, Q_{F} ; t, q\right)=\frac{\mathcal{Z}_{\text {oct }}^{\text {inst }}\left(\mathbf{Q}_{m}, Q_{F} ; t, q\right)}{\mathcal{M}\left(Q_{B_{1}}^{\prime}\right) \mathcal{M}\left(Q_{B_{2}}^{\prime} \frac{t}{q}\right)}
$$

The normalised partition function (9.12) has an enhanced $E_{5}$ symmetry [70], see also [53], and its 1-instanton term is given by

$$
\begin{aligned}
& \mathcal{Z}_{\text {norm }}^{1-\text { inst }}= \\
& \frac{q}{t} \frac{\left(1+\frac{q}{t}\right)\left(1+\sum_{\substack{i \neq j \\
i, j=1}}^{4} \frac{Q_{F}}{Q_{m_{i}} Q_{m_{j}}}+\frac{Q_{F}^{2}}{\prod_{k=1}^{4} Q_{m_{k}}}\right)-\sqrt{\frac{q}{t}}\left(1+Q_{F}\right) \sum_{i=1}^{4}\left(\frac{1}{Q_{m_{i}}}+\frac{Q_{F} Q_{m_{i}}}{\prod_{j=1}^{4} Q_{m_{j}}}\right)}{(1-q)\left(1-t^{-1}\right)\left(1-Q_{F} q / t\right)\left(1-Q_{F}^{-1} q / t\right)} .
\end{aligned}
$$

Notice this expression is symmetric under the interchange of all $Q_{m_{i}}$ for $i=1, \ldots, 4$. We can also present the result compactly at second order in the case of the unrefined partition function, where $q=t$, if we further set $q=e^{h}$ and take the limit where $h \rightarrow 0$. Then to leading order in $h$, the 2 -instanton term is

$$
\begin{aligned}
& (1-q)^{4} \mathcal{Z}_{\text {norm }}^{2-\text { inst }}= \\
& \quad \frac{1}{2}\left[\frac{Q_{F}}{\left(1-Q_{F}\right)^{2}}\left(2\left(1+\sum_{\substack{i \neq j \\
i, j=1}}^{4} \frac{Q_{F}}{Q_{m_{i}} Q_{m_{j}}}+\frac{Q_{F}^{2}}{\prod_{k=1}^{4} Q_{m_{k}}}\right)-\left(1+Q_{F}\right) \sum_{i=1}^{4}\left(\frac{1}{Q_{m_{i}}}+\frac{Q_{F} Q_{m_{i}}}{\prod_{j=1}^{4} Q_{m_{j}}}\right)\right]^{2} .\right.
\end{aligned}
$$

\section{2 $\mathrm{SU}(2)$ four-flavour $\mathcal{N}=1$ theory in $5 \mathrm{D}$ from gluing of $T_{2}$ blocks}

We now turn to the geometry depicted on the right side of figure 13 and the gluing of $T_{2}$ vertices. To look at this in a similar way, we first need the generalisation (2.27) of the $T_{2}$ partition function when two of the Young tableaux associated to external diagonal legs of the web are non-empty

$$
\begin{aligned}
\mathcal{Z}_{\vec{\lambda}}^{\text {top }, 2}\left(Q_{1}, Q_{2}, Q_{3} ; t, q\right)= & t^{\frac{\left\|\lambda_{1}^{t}\right\|^{2}+\left\|\lambda_{2}^{t}\right\|^{2}}{2}} \tilde{Z}_{\lambda_{1}^{t}}(q, t) \tilde{Z}_{\lambda_{2}^{t}}(q, t) \frac{\mathcal{M}\left(Q_{1} Q_{2} ; t, q\right)}{\mathcal{M}\left(Q_{1} \sqrt{\frac{t}{q}} ; t, q\right) \mathcal{M}\left(Q_{2} \sqrt{\frac{t}{q}} ; t, q\right)} \\
& \sum_{\nu}\left(Q_{3} \sqrt{\frac{q}{t}}\right)^{|\nu|} \frac{\mathcal{N}_{\lambda_{1} \nu}\left(Q_{1} \sqrt{\frac{t}{q}} ; t, q\right) \mathcal{N}_{\nu \lambda_{2}}\left(Q_{2} \sqrt{\frac{t}{q}} ; t, q\right)}{\mathcal{N}_{\nu \nu}(1 ; t, q) \mathcal{N}_{\lambda_{1} \lambda_{2}}\left(Q_{1} Q_{2} ; t, q\right)}
\end{aligned}
$$

Because we aim to use this as a building block when gluing, we keep here explicit the dependence on the $t, q$ deformation parameters. Recalling the discussion from section 2.2, when the external tableaux in (9.15) are empty the partition function reduces to

$$
\mathcal{Z}_{T_{2}}^{\text {top }}\left(Q_{1}, Q_{2}, Q_{3} ; t, q\right)=\frac{\mathcal{M}\left(Q_{1} Q_{2} ; t, q\right) \mathcal{M}\left(Q_{2} Q_{3} ; t, q\right) \mathcal{M}\left(Q_{1} Q_{3} \frac{t}{q} ; t, q\right)}{\mathcal{M}\left(Q_{1} Q_{2} Q_{3} \sqrt{\frac{t}{q}} ; t, q\right) \prod_{\ell=1}^{3} \mathcal{M}\left(Q_{\ell} \sqrt{\frac{t}{q}} ; t, q\right)} .
$$


It will be useful for us to write the topological strings partition function (9.15) for the $T_{2}$ web depicted in figure 7 in the following way, ${ }^{8}$ where it is normalized by $\mathcal{Z}_{T_{2}}^{\text {top }}(9.16)$

$$
\mathcal{Z}_{\lambda_{1}, \lambda_{2}}^{\text {top }, 2}\left(Q_{1}, Q_{2}, Q_{3} ; t, q\right)=\mathcal{Z}_{T_{2}}^{\text {top }}\left(Q_{1}, Q_{2}, Q_{3} ; t, q\right) \prod_{i=1}^{2} t^{\frac{\left\|\lambda_{i}^{t}\right\|^{2}}{2}} \tilde{Z}_{\lambda_{i}^{t}}(q, t) \frac{P_{\lambda_{1}, \lambda_{2}}\left(Q_{1}, Q_{2}, Q_{3} ; t, q\right)}{\mathcal{N}_{\lambda_{1} \lambda_{2}}\left(Q_{1} Q_{2} ; t, q\right)}
$$

and we have defined the function

$$
\begin{aligned}
P_{\lambda_{1}, \lambda_{2}}\left(Q_{1}, Q_{2}, Q_{3} ; t, q\right)= & \frac{\mathcal{M}\left(Q_{1} Q_{2} Q_{3} \sqrt{\frac{t}{q}} ; t, q\right) \mathcal{M}\left(Q_{3} \sqrt{\frac{t}{q}} ; t, q\right)}{\mathcal{M}\left(Q_{2} Q_{3} ; t, q\right) \mathcal{M}\left(Q_{1} Q_{3} \frac{t}{q} ; t, q\right)} \\
& \sum_{\nu}\left(Q_{3} \sqrt{\frac{q}{t}}\right)^{|\nu|} \frac{\mathcal{N}_{\lambda_{1} \nu}\left(Q_{1} \sqrt{\frac{t}{q}} ; t, q\right) \mathcal{N}_{\nu \lambda_{2}}\left(Q_{2} \sqrt{\frac{t}{q}} ; t, q\right)}{\mathcal{N}_{\nu \nu}(1 ; t, q)} .
\end{aligned}
$$

Let $\mathbf{Q}=\left(Q_{1}, Q_{2}, Q_{3}\right)$ denote a vector of Kähler parameters. The topological strings partition function obtained by gluing $T_{2}$ blocks, as depicted on the left of figure 13 , is

$$
\mathcal{Z}_{\text {hex }}\left(\mathbf{Q}, \mathbf{Q}^{\prime} ; t, q\right)=\sum_{\lambda_{1}, \lambda_{2}}\left(-Q_{B_{1}}\right)^{\left|\lambda_{1}\right|}\left(-Q_{B_{2}}\right)^{\left|\lambda_{2}\right|} \mathcal{Z}_{\lambda_{1}, \lambda_{2}}^{\text {top } 2}(\mathbf{Q} ; t, q) \mathcal{Z}_{\lambda_{2}^{t}, \lambda_{1}^{t}}^{\text {top } 2}\left(\mathbf{Q}^{\prime} ; q, t\right)
$$

To determine the first orders in the instanton expansion, we need to know explicitly the function $P_{\lambda_{1}, \lambda_{2}}\left(Q_{1}, Q_{2}, Q_{3} ; t, q\right)$ defined in equation (9.18) when the Young diagrams $\lambda_{1}, \lambda_{2}$ have one or two boxes. In the case with only one box, we find

$$
\begin{aligned}
& P_{\{1\}, \emptyset}\left(Q_{1}, Q_{2}, Q_{3} ; t, q\right)=1-Q_{1}\left(1+Q_{2} Q_{3}\right) \sqrt{\frac{q}{t}}+Q_{1} Q_{3}, \\
& P_{\emptyset,\{1\}}\left(Q_{1}, Q_{2}, Q_{3} ; t, q\right)=1-Q_{2}\left(1+Q_{1} Q_{3}\right) \sqrt{\frac{t}{q}}+Q_{2} Q_{3} .
\end{aligned}
$$

The first of these expressions has been previously computed by [70]. We have furthermore calculated

$$
\begin{aligned}
P_{\{2\}, \emptyset}(\mathbf{Q} ; t, q)= & 1+Q_{1} Q_{3}+\left(Q_{1} Q_{3}\right)^{2}+q Q_{1} Q_{3}\left(1+Q_{1} Q_{2} \frac{1}{t}\right)+Q_{1}^{2}\left(1+Q_{2} Q_{3}+\left(Q_{2} Q_{3}\right)^{2}\right) \frac{q^{2}}{t} \\
& -Q_{1}\left(1+Q_{1} Q_{3}\right)\left(1+Q_{2} Q_{3}\right) \sqrt{\frac{q}{t}}(1+q) \\
P_{\{1,1\}, \emptyset}(\mathbf{Q} ; t, q)= & 1+Q_{1} Q_{3}\left(1+\frac{1}{t}\right)+\left(Q_{1} Q_{3}\right)^{2}+Q_{1}^{2} \frac{q}{t}\left(Q_{2} Q_{3}+\frac{1}{t}\left(1+Q_{2} Q_{3}+\left(Q_{2} Q_{3}\right)^{2}\right)\right) \\
& -\sqrt{\frac{q}{t}} Q_{1}\left(1+\frac{1}{t}\right)\left(1+Q_{1} Q_{3}\right)\left(1+Q_{2} Q_{3}\right) \\
P_{\{1\},\{1\}}(\mathbf{Q} ; t, q)= & \left(1-Q_{1}\left(1+Q_{2} Q_{3}\right) \sqrt{\frac{q}{t}}+Q_{1} Q_{3}\right)\left(1-Q_{2}\left(1+Q_{1} Q_{3}\right) \sqrt{\frac{t}{q}}+Q_{2} Q_{3}\right) \\
& +Q_{1} Q_{2} Q_{3}\left(\sqrt{\frac{q}{t}}-\sqrt{q t}-\frac{1}{\sqrt{q t}}+\sqrt{\frac{t}{q}}\right)
\end{aligned}
$$

\footnotetext{
${ }^{8}$ One may note here that $\mathcal{N}_{\lambda_{1} \lambda_{2}}^{-1}\left(Q_{1} Q_{2} ; t, q\right)$ is half of the contribution of a $5 \mathrm{D}$ vector multiplet.
} 


$$
\begin{aligned}
P_{\emptyset,\{1,1\}}(\mathbf{Q} ; t, q)= & 1+Q_{2} Q_{3}(1+t)+\left(Q_{2} Q_{3}\right)^{2}+Q_{2}^{2} \frac{t}{q}\left(Q_{1} Q_{3}+t\left(1+Q_{1} Q_{3}+\left(Q_{1} Q_{3}\right)^{2}\right)\right) \\
& -\sqrt{\frac{t}{q}} Q_{2}(1+t)\left(1+Q_{1} Q_{3}\right)\left(1+Q_{2} Q_{3}\right) \\
P_{\emptyset,\{2\}}(\mathbf{Q} ; t, q)= & 1+Q_{2} Q_{3}+\left(Q_{2} Q_{3}\right)^{2}+\frac{1}{q} Q_{2} Q_{3}\left(1+Q_{1} Q_{2} t\right)+Q_{2}^{2}\left(1+Q_{1} Q_{3}+\left(Q_{1} Q_{3}\right)^{2}\right) \frac{t}{q^{2}} \\
& -Q_{2}\left(1+Q_{1} Q_{3}\right)\left(1+Q_{2} Q_{3}\right) \sqrt{\frac{t}{q}}\left(1+\frac{1}{q}\right) .
\end{aligned}
$$

If we now apply the following mapping

$$
Q_{m_{3}}=Q_{1}, \quad Q_{m_{1}}=Q_{1}^{\prime}, \quad Q_{m_{2}}=\frac{1}{Q_{3}}, \quad Q_{m_{4}}=\frac{1}{Q_{3}^{\prime}}, \quad Q_{F}=Q_{1} Q_{2}=Q_{1}^{\prime} Q_{2}^{\prime}
$$

and set the base Kähler parameters to be

$$
Q_{B_{1}}=u \frac{Q_{F}}{Q_{m_{1}}}, \quad Q_{B_{2}}=u \frac{Q_{F}}{Q_{m_{3}}}
$$

then the partition function (9.4) can be factored to

$$
\mathcal{Z}_{\text {hex }}=\mathcal{Z}_{\text {hex }}^{\text {pert }} \mathcal{Z}_{\text {hex }}^{\text {inst }}, \quad \text { where } \quad \mathcal{Z}_{\text {hex }}^{\text {pert }}\left(\mathbf{Q}, \mathbf{Q}^{\prime} ; t, q\right)=\mathcal{Z}_{T_{2}}^{\text {top }}(\mathbf{Q} ; t, q) \mathcal{Z}_{T_{2}}^{\text {top }}\left(\mathbf{Q}^{\prime} ; q, t\right)
$$

and the instanton part has an expansion in the instanton parameter $u$. The first order term in this expansion is

$$
\begin{aligned}
u \mathcal{Z}_{\text {hex }}^{1-\text { inst }}=\sqrt{q t} \tilde{Z}_{\{1\}}(q, t) \tilde{Z}_{\{1\}}(t, q)( & -Q_{B_{1}} \frac{P_{\{1\}, \emptyset}(\mathbf{Q} ; t, q) P_{\emptyset,\{1\}}\left(\mathbf{Q}^{\prime} ; q, t\right)}{\mathcal{N}_{\{1\}, \emptyset}\left(Q_{1} Q_{2} ; t, q\right) \mathcal{N}_{\emptyset,\{1\}}\left(Q_{1}^{\prime} Q_{2}^{\prime} ; q, t\right)} \\
& \left.-Q_{B_{2}} \frac{P_{\emptyset,\{1\}}(\mathbf{Q} ; t, q) P_{\{1\}, \emptyset}\left(\mathbf{Q}^{\prime} ; q, t\right)}{\mathcal{N}_{\emptyset,\{1\}}\left(Q_{1} Q_{2} ; t, q\right) \mathcal{N}_{\{1\}, \emptyset}\left(Q_{1}^{\prime} Q_{2}^{\prime} ; q, t\right)}\right)
\end{aligned}
$$

and it becomes

$$
\begin{aligned}
& \mathcal{Z}_{\text {hex }}^{1-\text { inst }}= \\
& \frac{q}{t} \frac{\left(1+\frac{q}{t}\right)\left(1+\sum_{\substack{i \neq j \\
i, j=1}}^{4} \frac{Q_{F}}{Q_{m_{i}} Q_{m_{j}}}+\frac{Q_{F}^{2}}{\prod_{k=1}^{4} Q_{m_{k}}}\right)-\sqrt{\frac{q}{t}}\left(1+Q_{F}\right) \sum_{i=1}^{4}\left(\frac{1}{Q_{m_{i}}}+\frac{Q_{F} Q_{m_{i}}}{\prod_{j=1}^{4} Q_{m_{j}}}\right)}{(1-q)\left(1-t^{-1}\right)\left(1-Q_{F} q / t\right)\left(1-Q_{F}^{-1} q / t\right)} .
\end{aligned}
$$

Notice this is identical to equation (9.13), expressing the 1-instanton term in the normalised partition function derived by gluing horizontally strip geometries. We can repeat this calculation at second order of the instanton expansion, where the pairs of Young tableaux $\lambda_{1}, \lambda_{2}$ which need to be considered are

$$
(\{2\}, \emptyset), \quad(\{1,1\}, \emptyset), \quad(\{1\},\{1\}), \quad(\emptyset,\{1,1\}), \quad(\emptyset,\{2\}) .
$$


Taking the terms corresponding to these Young diagrams from equation (9.4) gives

$$
\begin{aligned}
u^{2} \mathcal{Z}_{\text {hex }}^{2-\text { inst }}= & Q_{B_{1}} Q_{B_{2}} q t\left(\tilde{Z}_{\{1\}}(q, t) \tilde{Z}_{\{1\}}(t, q)\right)^{2} \frac{P_{\{1\},\{1\}}(\mathbf{Q} ; t, q) P_{\{1\},\{1\}}\left(\mathbf{Q}^{\prime} ; q, t\right)}{\mathcal{N}_{\{1\},\{1\}}\left(Q_{F} ; t, q\right) \mathcal{N}_{\{1\},\{1\}}\left(Q_{F} ; q, t\right)} \\
& +Q_{B_{1}}^{2} t q^{2} \tilde{Z}_{\{1,1\}}(q, t) \tilde{Z}_{\{2\}}(t, q) \frac{P_{\{2\}, \emptyset}(\mathbf{Q} ; t, q) P_{\emptyset,\{1,1\}}\left(\mathbf{Q}^{\prime} ; q, t\right)}{\mathcal{N}_{\{2\}, \emptyset}\left(Q_{F} ; t, q\right) \mathcal{N}_{\emptyset,\{1,1\}}\left(Q_{F} ; q, t\right)} \\
& +Q_{B_{1}}^{2} t^{2} q \tilde{Z}_{\{2\}}(q, t) \tilde{Z}_{\{1,1\}}(t, q) \frac{P_{\{1,1\}, \emptyset}(\mathbf{Q} ; t, q) P_{\emptyset,\{2\}}\left(\mathbf{Q}^{\prime} ; q, t\right)}{\mathcal{N}_{\{1,1\}, \emptyset}\left(Q_{F} ; t, q\right) \mathcal{N}_{\emptyset,\{2\}}\left(Q_{F} ; q, t\right)} \\
& +Q_{B_{2}}^{2} t q^{2} \tilde{Z}_{\{1,1\}}(q, t) \tilde{Z}_{\{2\}}(t, q) \frac{P_{\emptyset,\{2\}}(\mathbf{Q} ; t, q) P_{\{1,1\}, \emptyset}\left(\mathbf{Q}^{\prime} ; q, t\right)}{\mathcal{N}_{\emptyset,\{2\}}\left(Q_{F} ; t, q\right) \mathcal{N}_{\{1,1\}, \emptyset}\left(Q_{F} ; q, t\right)} \\
& +Q_{B_{2}}^{2} t^{2} q \tilde{Z}_{\{2\}}(q, t) \tilde{Z}_{\{1,1\}}(t, q) \frac{P_{\emptyset,\{1,1\}}(\mathbf{Q} ; t, q) P_{\{2\}, \emptyset}\left(\mathbf{Q}^{\prime} ; q, t\right)}{\mathcal{N}_{\emptyset,\{1,1\}}\left(Q_{F} ; t, q\right) \mathcal{N}_{\{2\}, \emptyset}\left(Q_{F} ; q, t\right)}
\end{aligned}
$$

Substituting appropriately the functions (9.21), going to the unrefined case where $t=q$, and further setting $q=e^{h}$ for simplicity, gives to leading order in $h$ in the limit $h \rightarrow 0$

$$
\begin{aligned}
& (1-q)^{4} \mathcal{Z}_{\text {hex }}^{2-\text { inst }}= \\
& \frac{1}{2}\left[\frac{Q_{F}}{\left(1-Q_{F}\right)^{2}}\left(2\left(1+\sum_{\substack{i \neq j \\
i, j=1}}^{4} \frac{Q_{F}}{Q_{m_{i}} Q_{m_{j}}}+\frac{Q_{F}^{2}}{\prod_{k=1}^{4} Q_{m_{k}}}\right)-\left(1+Q_{F}\right) \sum_{i=1}^{4}\left(\frac{1}{Q_{m_{i}}}+\frac{Q_{F} Q_{m_{i}}}{\prod_{j=1}^{4} Q_{m_{j}}}\right)\right]^{2} .\right.
\end{aligned}
$$

We therefore note that, like in the case of the 1-instanton term (9.26), the 2-instanton coefficient is also identical to its counterpart from the previous subsection (9.14) calculated from the horizontal gluing of strip geometries. Therefore at leading orders in the instanton expansion, we have identified

$$
\left[\mathcal{M}\left(Q_{B_{1}}^{\prime}\right) \mathcal{M}\left(Q_{B_{2}}^{\prime} t / q\right)\right]^{-1} \mathcal{Z}_{\text {oct }}^{\text {inst }}=\mathcal{Z}_{\text {norm }}^{\text {inst }}=\mathcal{Z}_{\text {hex }}^{\text {inst }}
$$

from equations (9.12) and respectively (9.24). This result is highly non-trivial and supports the slicing invariance conjecture [21] whereby, under a correct identification of parameters, the instanton expansions computed for vertical and diagonal slicing of a web diagram are identical.

\subsection{Mapping to Virasoro four point conformal blocks}

It is known that the instanton expansion in equation (9.9) can be mapped to the expansion of Virasoro four point conformal blocks in powers of the conformal cross-ratio [1]. Therefore, via equations (9.12) and (9.29), one would naturally expect to be able to also map the coefficients of the instanton expansion (9.24) derived from gluing $T_{2}$ blocks to the same expansion on the side of conformal field theory. Following the analysis from section 4 which related the $T_{2}$ Kähler parameters to Liouville CFT momenta, we repeat the process for the two $T_{2}$ vertices glued to form the web on the right of figure 13. We thus identify the following dictionary

$$
Q_{1}=q^{-\beta\left(\frac{Q}{2}+a_{1}-a_{2}+a\right)}, \quad Q_{2}=q^{-\beta\left(\frac{Q}{2}-a_{1}+a_{2}+a\right)}, \quad Q_{3}=q^{\beta\left(\frac{3 Q}{2}+a_{1}+a_{2}+a\right)},
$$


where $Q=\beta^{-1}-\beta$ and $a_{1}=a_{2}+a+\beta s$ and similarly

$$
Q_{1}^{\prime}=q^{-\beta\left(\frac{Q}{2}+a_{3}-a_{4}+a\right)}, \quad Q_{2}^{\prime}=q^{-\beta\left(\frac{Q}{2}-a_{3}+a_{4}+a\right)}, \quad Q_{3}^{\prime}=q^{\beta\left(\frac{3 Q}{2}+a_{3}+a_{4}+a\right)} .
$$

Taking this dictionary together with the map (9.22) and the relation $q^{\beta}=e^{R}$, in the limit $R \rightarrow 0$, equation (9.10) becomes

$$
Z_{n}^{1-\text { inst }}=-\frac{\left(\Delta_{a}-\Delta_{a_{1}}+\Delta_{a_{2}}\right)\left(\Delta_{a}-\Delta_{a_{3}}+\Delta_{a_{4}}\right)}{2 \Delta_{a}}+2 a_{1}\left(a_{3}+Q\right),
$$

with $\Delta_{a}=a(a+Q)$. This can be compared to the instanton expansion in [2], whereby the term $2 a_{1}\left(a_{3}+Q\right)$ is identified with the $\mathrm{U}(1)$ factor of [1] while the first term appears in the expansion of the four point conformal block of Liouville CFT.

\section{Conclusions and outlook}

\subsection{Using the $T_{2}$-vertex in the AGT-correspondence}

We have presented direct evidence for the conjecture that gluing $T_{2}$-vertices yields partition functions related to instanton partition functions, or equivalently Liouville conformal blocks, in the four-dimensional limit. This is not completely unexpected in view of the relations between the $T_{2}$ vertex and strip discussed in section 6 , given that such results are well-known in the case of the strip. However, on first sight one finds results for the $T_{2}$ vertex and strip differing by a non-trivial factor. One needs to take into account the fact that both partition functions represent piecewise analytic functions on their respective domains of definition in a common parameter space. Agreement is found when comparing the results for the chambers in which both are defined. It should also be noted that the strip partition functions have a smaller domain of definition than the $T_{2}$ partition functions, indicating that the relation between the two types of vertices is somewhat subtle.

It would be nice to find a mathematical proof of the conjectured relation between gluing of $T_{2}$ vertices and instanton partition functions. One should also notice that the $T_{2}$ vertex offers more options for gluing than the strip. This additional flexibility should be useful for the investigations of the so-called Sicilian theories [71].

Our results indicate that the geometric engineering of $4 d$ field theories determines the one-loop contributions to the instanton partition functions up to leg factors. The result can easily be summarised as follows. The arguments of the Barnes double Gamma functions appearing in the one-loop contributions should always be chosen in such a way that the partition functions are analytic within the whole region in the parameter space under consideration. This requirement fixes the arguments of the Barnes double Gamma functions uniquely, reproducing the results we had carefully derived by various methods before.

One should note that the rule formulated above, natural as it may be, implies that the $T_{2}$ partition functions are only piecewise analytic over the parameter space. The loci where analyticity fails are related to the walls separating different Kähler cones related by flop transitions within the extended Kähler moduli space.

A direct field theoretic explanation of the piecewise analyticity does not seem to be available at the moment. One may note, however, that the instanton partition functions 
admit a physical interpretation as hemisphere partition functions [72, 73]. It seems quite possible that the precise form of the one-loop contributions depends in a subtle way on the boundary conditions defining the hemisphere partition functions. Another possible approach could be to follow the approach of $[38,39]$ relating the relevant $5 d$ partition functions to partition functions of a three-dimensional gauge theory. The chamber dependence discussed in this paper is somewhat reminiscent of the Stokes phenomena studied in the context of $3 d$ superconformal field theories on (deformed) hemispheres in [74, 75]. It would be interesting if similar phenomena could explain our results more directly in field theoretical terms.

The one-loop contributions to the instanton partition functions are related by the AGTcorrespondence to the choice of a basis in the one-dimensional space of conformal blocks on the three-punctures sphere. The geometric engineering of the $4 d$ field theories of interest therefore refines the previous results on the AGT-correspondence in an interesting way by selecting preferred bases for the spaces of three-point conformal blocks. The relation of the geometric parameters of the topological string theory to the parameters used in Liouville theory derived in sections 4 and 5 involved a slightly non-obvious sign which was explained by the subtleties in the relation between geometric transitions and matrix model representation for the partition functions discussed in section 7.2.

\subsection{Higher rank}

As we have already discussed in the introduction, the problems we encounter in this current

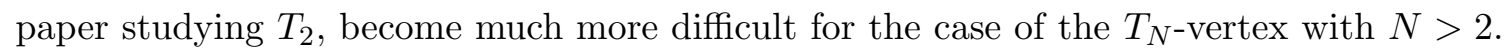
In the case of the $W_{N}$-algebras with $N>2$ one finds an infinite-dimensional space of chiral vertex operators in general. As discussed in [68, 69], one may expect that useful bases for this space can be constructed with the help of the free field representation. The powers of the screening charges provide labels for the elements of this basis. However, such bases are not unique, different choices of the contours used to define the screening charges, or equivalently, different choices of the ordering of these objects define different bases for the spaces of conformal blocks. As it is possible to consider continuous powers of the screening charges, as first observed in [76] for $N=2$, one can define bases labelled by continuous parameters in this way. ${ }^{9}$

Considering $T_{N}$-vertices for $N \geq 3$ one finds a qualitatively similar picture in the sense that one can certainly define families of operators $\mathcal{V}_{\mathbf{u}_{3}, \mathbf{u}_{1}}^{\mathbf{u}_{2}, \mathbf{w}}(1)$ via (8.7) depending on the same number $\frac{1}{2}(N-2)(N-1)$ of parameters, now identified with the collection $\mathbf{w}$ of widths of the faces of the toric diagram in figure 14. One might hope that the operators defined from the $T_{N}$-partition functions via (8.7) indeed have a limit $q \rightarrow 1$ representing vertex operators of the $W_{N}$-algebra.

However, at the moment it is not even clear if this limit exists at all, in general. Even less clear is if the limit, assuming it exists, is related to a vertex operator of the $W_{N}$-algebra.

\footnotetext{
${ }^{9}$ It is not easy to extend the well-known results for positive integer powers of the screening charges to continuous values of these parameters. Reconstructing a meromorphic function from it's residues is a nontrivial problem as there is the freedom to multiply with an entire function, in general. Moreover, without invoking the duality $b \leftrightarrow b^{-1}$ it is difficult to find the full set of poles and residues the final answer has.
} 
One would need to check that the Ward identities of the $W_{N}$ symmetry are satisfied. And it is furthermore unclear if the putative limit will be one of the bases proposed in $[68,69]$.

The free field representation simplifies considerably when the powers of the screening charges are all positive integers. There is in fact a nice correspondence with a similar simplification in the topological strings partition function for the $T_{N}$-diagram, occurring when the Kähler parameters satisfy certain integrality conditions, leading to integral formulae resembling the expressions from the free field representation of the $W_{N}$-algebra [39]. As a first step towards the identification with vertex operators of the $W_{N}$-algebra we have worked out the relation between the Kähler parameters and the numbers of screening operators in the resulting expressions. The map from the Kähler parameters associated to a $T_{N}$ web diagram to the momenta $a_{1}, a_{2}, a_{3}$ of Toda three point conformal blocks is as follows

$$
\frac{P_{1}^{(a)}}{P_{1}^{(a+1)}}=\frac{q}{t} q^{\beta\left(a_{1}, e_{a}\right)}, \quad \frac{P_{2}^{(a)}}{P_{2}^{(a+1)}}=\frac{t}{q} q^{-\beta\left(a_{2}, e_{a}\right)}, \quad \frac{P_{3}^{(N-a)}}{P_{3}^{(N-a+1)}}=\frac{q}{t} q^{\beta\left(a_{3}, e_{a}\right)} .
$$

Here $e_{a}$ are the simple roots of the Lie algebra $s l_{N}$, with $a=1, \ldots, N-1$ and $($,$) the$ inner product $\left(e_{a}, e_{b}\right)=\kappa_{a b}$, where $\kappa_{a b}$ is the Cartan matrix. The momenta are related as

$$
a_{3}=a_{1}+a_{2}+\beta \sum_{k=1}^{N-1} s_{k} e_{k},
$$

with screening numbers $s_{k}$ that are partitioned as $s_{k}=\sum_{i=1}^{k} \sum_{j=k}^{N-1} s_{i j}$. The relation to the Kähler parameters in figure 14 is

$$
\beta s_{i j}=-\frac{1}{R} \ln \left(Q_{l ; j-i+1}^{(i)}\right)+\frac{Q}{2},
$$

the derivation ${ }^{10}$ of which can be found in [77]. Through (10.3) we discover the relation between the $(N-1)(N-2) / 2$ Coulomb moduli of the $T_{N}$ theories and the $(N-1)(N-2) / 2$ composite screening charges of the free field representation which label a basis in the space of conformal blocks on $C_{0,3}$.

However, it is not clear to us, at the moment, how to take the limit $q \rightarrow 1$ using the contours of integration considered in [39]. Great care is needed to handle the possibility of contours getting pinched between poles of the integrand which collide in the limit $q \rightarrow 1$. We plan to return to this issue in future work.

\section{Acknowledgments}

The authors would like to thank Sara Pasquetti for useful discussions.

The work of I. Coman and J. Teschner is supported by the Deutsche Forschungsgemeinschaft (DFG) through the collaborative Research Centre SFB 676 "Particles, Strings

\footnotetext{
${ }^{10}$ The relations between the Kähler parameters associated to internal edges of the $T_{N}$ diagram and the momenta parameters of Toda CFT are insensitive to the change in CFT conventions between those used in section 4 and the ones from [77]. The derivation of these relations in section 19 of [77] therefore carries through.
} 


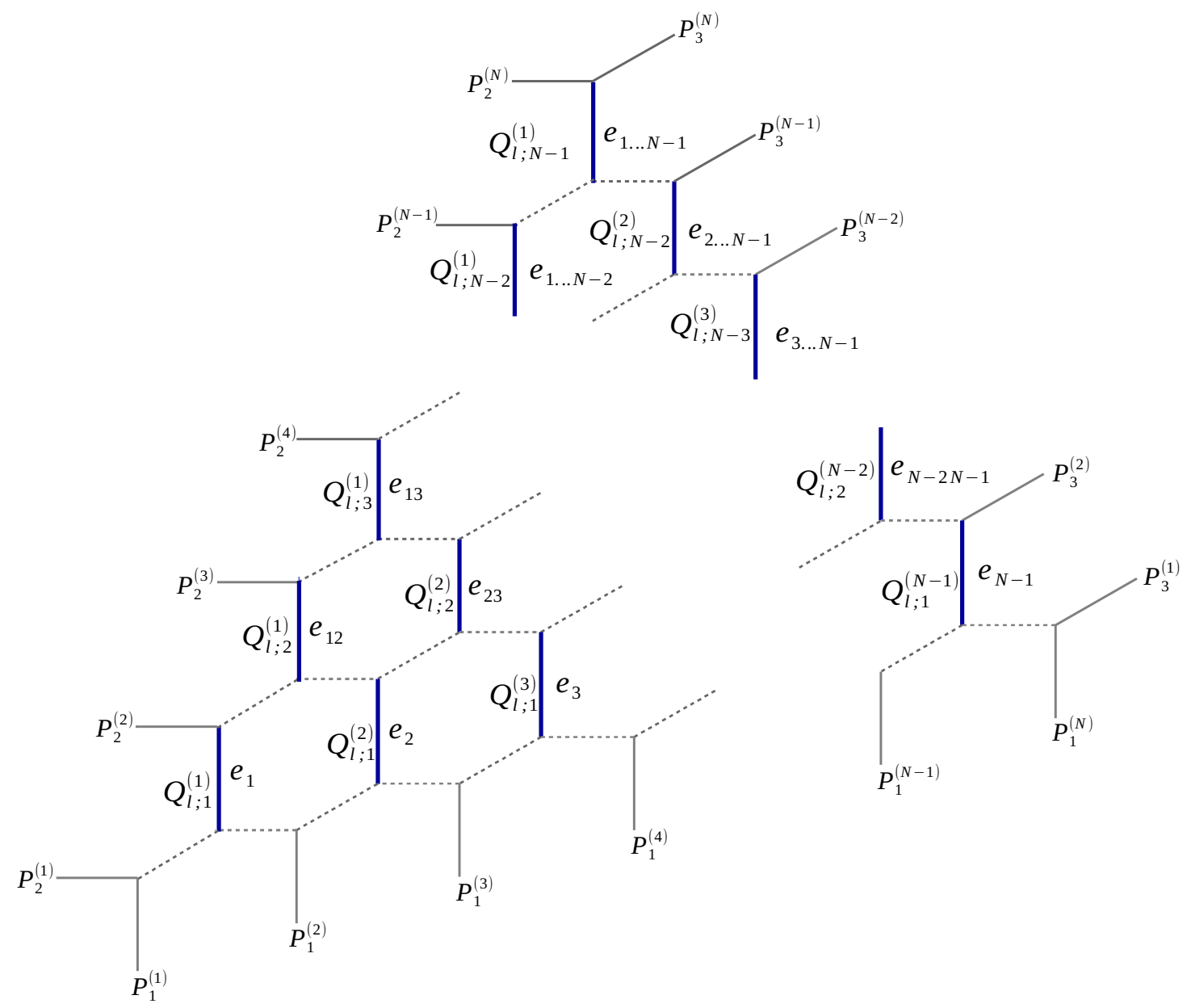

Figure 14. $T_{N}$ web diagram with an assignment of Kähler parameters to the inner vertical segments, the positions of the external semi-infinite branes, and the assignment among the Kähler parameters $Q_{l ; q}^{(p)}$ and positive roots $e_{i j}=\sum_{k=i}^{j} e_{k}$, for $e_{k}$ simple roots of the Lie algebra $s l_{N}$, $k=1, \ldots, N-1$.

and the Early Universe", project A10. The work of I. Coman is also supported by the ERC starting grant H2020 ERC StG No.640159. E. Pomoni's work is supported by the German Research Foundation (DFG) via the Emmy Noether program "Exact results in Gauge theories".

\section{A Special functions}

Here we collect definitions and identities for special functions. The quantum dilogarithm

$$
\varphi(x)=(x ; q)_{\infty}=\prod_{n=0}^{\infty}\left(1-q^{n} x\right), \quad|q|<1
$$

is a special case of shifted factorial function

$$
\left(x ; q_{1}, \ldots, q_{r}\right)_{\infty}=\prod_{n_{1}, \ldots, n_{r}=0}^{\infty}\left(1-x q_{1}^{n_{1}} \cdots q_{r}^{n_{r}}\right), \quad \forall \quad\left|q_{i}\right|<1 .
$$


A definition that overcomes the need to specify a regime of the $\left|q_{i}\right|$ and resolves issues of convergence is the polylogarithm function or the plethystic exponential

$$
\left(x ; q_{1}, \ldots, q_{r}\right)_{\infty}=\exp \left(-\mathrm{Li}_{r+1}(x)\right), \quad|x|<1
$$

with the definition

$$
\operatorname{Li}_{r}(z)=\sum_{n=1}^{\infty} \frac{z^{n}}{n^{r}}, \quad|z|<1
$$

for all complex arguments $z$ with $|z|<1$.

The infinite product function $\mathcal{M}(U) \equiv \mathcal{M}(U ; t, q)$ which appears often in the main text is also defined as a shifted factorial

$$
\mathcal{M}(U ; t, q)=(U q ; t, q)_{\infty}^{-1}= \begin{cases}\prod_{i, j=1}^{\infty}\left(1-U t^{i-1} q^{j}\right)^{-1} & \text { for }|t|<1,|q|<1 \\ \prod_{i, j=1}^{\infty}\left(1-U t^{i-1} q^{1-j}\right) & \text { for }|t|<1,|q|>1 \\ \prod_{i, j=1}^{\infty}\left(1-U t^{-i} q^{j}\right) & \text { for }|t|>1,|q|<1 \\ \prod_{i, j=1}^{\infty}\left(1-U t^{-i} q^{1-j}\right)^{-1} & \text { for }|t|>1,|q|>1\end{cases}
$$

converging for all $U$. This function can be alternatively written as a plethystic exponential

$$
\mathcal{M}(U ; t, q)=\exp \left[\sum_{m=1}^{\infty} \frac{U^{m}}{m} \frac{q^{m}}{\left(1-t^{m}\right)\left(1-q^{m}\right)}\right],
$$

which converges for all $t$ and all $q$ provided that $|U|<q^{-1+\theta(|q|-1)} t^{\theta(|t|-1)}$, where only here $\theta(x)$ denotes the step function which is $\theta(x)=1$ if $x>0$ and $\theta(x)=0$ if $x \leq 0$. From the analytic properties of the shifted factorials (A.2) we can derive the identities

$$
\mathcal{M}\left(U ; t^{-1}, q\right)=\frac{1}{\mathcal{M}(U t ; t, q)}, \quad \mathcal{M}\left(U ; t, q^{-1}\right)=\frac{1}{\mathcal{M}\left(U q^{-1} ; t, q\right)},
$$

as well as the following functional relations

$$
\mathcal{M}(U t ; t, q)=(U q ; q)_{\infty} \mathcal{M}(U ; t, q), \quad \mathcal{M}(U q ; t, q)=(U q ; t)_{\infty} \mathcal{M}(U ; t, q) .
$$

A further useful identity is

$$
\mathcal{M}(Q ; q, t)=\mathcal{M}\left(Q \frac{t}{q} ; t, q\right)
$$

Nekrasov partition function. The quantum dilogarithm enters the definition of the Nekrasov partition function [22] through the functions $\mathcal{N}_{R P}$

$$
\mathcal{N}_{R P}(Q ; t, q)=\prod_{i=1}^{\infty} \prod_{j=1}^{\infty} \frac{\varphi\left(Q q^{R_{i}-P_{j}+1} t^{j-i}\right)}{\varphi\left(Q q^{R_{i}-P_{j}+1} t^{j-i-1}\right)} \frac{\varphi\left(Q q t^{j-i-1}\right)}{\varphi\left(Q q t^{j-i}\right)}
$$

which is equivalent to

$$
\mathcal{N}_{R P}(Q ; t, q)=\prod_{(i, j) \in R}\left(1-Q q^{R_{i}-j+1} t^{P_{j}^{t}-i}\right) \prod_{(i, j) \in P}\left(1-Q q^{-P_{i}+j} t^{-R_{j}^{t}+i-1}\right)
$$


and also to

$$
\mathcal{N}_{R P}(Q ; t, q)=\prod_{(i, j) \in P}\left(1-Q q^{R_{i}-j+1} t^{P_{j}^{t}-i}\right) \prod_{(i, j) \in R}\left(1-Q q^{-P_{i}+j} t^{-R_{j}^{t}+i-1}\right) .
$$

In the main text, we label $\mathcal{N}_{R P}(Q ; t, q) \equiv \mathcal{N}_{R P}(Q)$ in order not to make our formulas too baroque. The notation in these expressions is as follows: $R$ and $P$ are Young tableaux or partitons, $R_{i}$ represents the length of row $i$ and $R^{t}$ is the dual partition to $R$, with rows and column exchanged with respect to those of $R$. Furthermore, a box $s \in R$ on row $i$ and column $j$ of $R$ has leg-length $l_{R}(s)=R_{j}^{t}-i$. When one of the partitions in definition (A.11) is empty, this becomes one of the following

$$
\begin{aligned}
& \mathcal{N}_{\emptyset R}(Q)=\prod_{(i, j) \in R}\left(1-Q q^{-R_{i}+j} t^{i-1}\right)=\prod_{i=1}^{N_{R}} \frac{\varphi\left(Q q^{-R_{i}+1} t^{i-1}\right)}{\varphi\left(Q q t^{i-1}\right)}, \\
& \mathcal{N}_{R \emptyset}(Q)=\prod_{(i, j) \in R}\left(1-Q q^{R_{i}-j+1} t^{-i}\right)=\prod_{i=1}^{N_{R}} \frac{\varphi\left(Q q t^{-i}\right)}{\varphi\left(Q q^{R_{i}+1} t^{-i}\right)}
\end{aligned}
$$

Note here for future reference that for $Q=v^{-2} t^{N}$, the partition function

$$
\mathcal{N}_{R \emptyset}(Q)=\prod_{i=1}^{N_{R}} \frac{\varphi\left(Q q t^{-i}\right)}{\varphi\left(Q q^{R_{i}+1} t^{-i}\right)}
$$

vanishes if $N_{R}>N$; the argument for the quantum dilogarithm in the numerator $\varphi\left(Q q t^{-i}\right)$ becomes 1 at $i=N+1$ and the numerator vanishes. Similarly, $\mathcal{N}_{\emptyset R}(Q)$ vanishes if $Q=t^{-N}$ and $N_{R}>N$. This follows from the identity

$$
\mathcal{N}_{R P}\left(Q v^{-2}\right)=\mathcal{N}_{P R}\left(Q^{-1}\right)\left(Q v^{-1}\right)^{|R|+|P|} \frac{f_{R}}{f_{P}},
$$

where

$$
f_{R}=\prod_{(i, j) \in R}(-1) q^{R_{i}-j+1 / 2} t^{-R_{j}^{t}+i-1 / 2}=(-1)^{|R|} q^{\|R\|^{2} / 2} t^{-\left\|R^{t}\right\|^{2} / 2}
$$

for $|R|=\sum_{i} R_{i}$ and $\|R\|^{2}=\sum_{i} R_{i}^{2}$. Finally, note that when the partitions $R$ and $P$ are finite, whereby the Young tableau have a finite number of rows, equation (A.10) becomes

$$
\mathcal{N}_{R P}(Q)=\prod_{i=1}^{N_{R}} \prod_{j=1}^{N_{P}} \frac{\varphi\left(Q q^{R_{i}-P_{j}+1} t^{j-i}\right)}{\varphi\left(Q q^{R_{i}-P_{j}+1} t^{j-i-1}\right)} \frac{\varphi\left(Q q t^{j-i-1}\right)}{\varphi\left(Q q t^{j-i}\right)} \mathcal{N}_{R \emptyset}\left(t^{N_{P}} Q\right) \mathcal{N}_{\emptyset P}\left(t^{-N_{R}} Q\right)
$$

Similarly to (A.13)-(A.14), the partition function (A.18) also vanishes when $Q=v^{-2} t^{N}$ unless $N_{R}-N_{P} \leq N$ (and likewise when $Q=t^{-N}$ ). The function $\mathcal{N}_{R P}$ further satisfies the identity

$$
\mathcal{N}_{R P}(Q ; q, t)=\mathcal{N}_{P^{t} R^{t}}\left(Q \frac{t}{q} ; t, q\right)
$$


Special functions for topological string amplitudes. When writing topological string amplitudes, the following two functions are ubiquitous. Firstly, the function

$$
\tilde{Z}_{\nu}(t, q)=\prod_{i=1}^{\ell(\nu)} \prod_{j=1}^{\nu_{i}}\left(1-t^{\nu_{j}^{t}-i+1} q^{\nu_{i}-j}\right)^{-1}
$$

which up to an overall coefficient is the principal specialisation of the Macdonald function and secondly, the infinite product

$$
\mathcal{R}_{\lambda \mu}(Q ; t, q)=\prod_{i, j=1}^{\infty}\left(1-Q t^{i-\frac{1}{2}-\lambda_{j}} q^{j-\frac{1}{2}-\mu_{i}}\right)=\mathcal{M}\left(Q \sqrt{\frac{t}{q}} ; t, q\right)^{-1} \mathcal{N}_{\lambda^{t} \mu}\left(Q \sqrt{\frac{t}{q}} ; t, q\right)
$$

The function (A.20) is related to (A.10) through

$$
\mathcal{N}_{\mu \mu}(1 ; t, q)=\left(-\sqrt{\frac{q}{t}}\right)^{|\mu|} t^{-\frac{\left\|\mu^{t}\right\|^{2}}{2}} q^{-\frac{\|\mu\|^{2}}{2}}\left(\tilde{Z}_{\mu}(t, q) \tilde{Z}_{\mu^{t}}(q, t)\right)^{-1} .
$$

\section{A.1 The limit $q \rightarrow 1$}

In this subsection we study how to take the $q \rightarrow 1$ of $\varphi(z)$ and $\mathcal{M}(z)$. We begin with the quantum dilogarithm $\varphi(z)$ and its plethystic exponential expression

$$
\varphi(z)=(z ; q)_{\infty}=\exp \left[-\sum_{m=1}^{\infty} \frac{z^{m}}{m} \frac{1}{\left(1-q^{m}\right)}\right],
$$

using which we can derive

$$
\log \varphi(z)=\frac{1}{\log q} \operatorname{Li}_{2}(z)+\frac{1}{2} \log (1-z)+\mathcal{O}(\log q) .
$$

This identity was also derived form Kirillov in [78]. Observe that by forming the ratio $\frac{\varphi(q)}{\varphi(z)}$ we eliminate the leading $\mathrm{Li}_{2}$ divergence in the $q \rightarrow 1$ limit and then by further dividing by the subleading log term we obtain a function

$$
\Gamma_{q}(z)=\frac{\varphi(q)}{\varphi\left(q^{z}\right)}(1-q)^{1-z}
$$

that is finite in the $q \rightarrow 1$ limit

$$
\lim _{q \rightarrow 1} \Gamma_{q}(z)=\exp \left(\zeta(0)(1-x)(\log q)+\mathcal{O}(\log q)^{2}\right)
$$

with $\zeta(0)=-\frac{1}{2}$. This is a $q$-deformed version of the usual Gamma function studied by many authors and satisfies the functional relation

$$
\Gamma_{q}(z+1)=\frac{1-q^{z}}{1-q} \Gamma_{q}(z)=[z]_{q} \Gamma_{q}(z) .
$$

It is proven by Koornwinder in the appendix B of [79] that

$$
\lim _{q \rightarrow 1} \Gamma_{q}(z)=\Gamma(z) .
$$


We may further compute the $q \rightarrow 1$ limit of

$$
\log (\varphi(t) / \varphi(q))=-\left(b^{2}+1\right) \zeta(1)+\mathcal{O}(\log q),
$$

using (A.23), although it is better to do so using

$$
\lim _{q \rightarrow 1}(\varphi(t) / \varphi(q))=\left(\Gamma\left(-b^{2}\right)\right)^{-1}(1-q)^{1+b^{2}} .
$$

Finally, we can derive the following useful identity for the limit of the ratio of quantum dilogarithm functions

$$
\lim _{q \rightarrow 1} \frac{\varphi\left(q^{\alpha_{1}} x\right)}{\varphi\left(q^{\alpha_{2}} x\right)}=(1-x)^{\alpha_{2}-\alpha_{1}},
$$

using at an intermediate step the identity

$$
\operatorname{Li}_{2}(x)=-\int_{0}^{x} \frac{d y}{y} \log (1-y) .
$$

We can now proceed similarly with the $\mathcal{M}$ function using (A.6)

$$
\mathcal{M}(z)=(z q ; t, q)_{\infty}^{-1}=\exp \left[\sum_{m=1}^{\infty} \frac{z^{m}}{m} \frac{q^{m}}{\left(1-t^{m}\right)\left(1-q^{m}\right)}\right],
$$

and in the $q \rightarrow 1$ limit we obtain

$$
b^{2} \log (z ; q, t)_{\infty}=-\frac{\operatorname{Li}_{3}(z)}{(\log q)^{2}}-\frac{1}{2} \frac{\operatorname{Li}_{2}(z)}{\log q}\left(b^{2}-1\right)+\frac{\operatorname{Li}_{1}(z)}{3 !} \frac{b^{4}-3 b^{2}+1}{2}+\mathcal{O}(\log q) .
$$

We find that the function

$$
\Gamma_{q, t}(x)=\frac{\mathcal{M}(t / q)}{\mathcal{M}\left(t^{x} / q\right)} \varphi(q)^{x-1}(1-q)^{\frac{1}{2}(x-1)\left(2-b^{-2} x\right)}
$$

has a finite $q \rightarrow 1$ limit and it furthermore satisfies, using (A.8), the functional relation

$$
\Gamma_{q, t}(x+1)=\Gamma_{q}(x) \Gamma_{q, t}(x) .
$$

\section{B Variants of Jackson integrals and the $q \rightarrow 1$ limit}

Equation (5.7) is useful for studying the limit $q \rightarrow 1$ of integrals encountered in section 5 . The Jackson integral (5.7), which we reproduce here,

$$
\mathcal{I}_{q}^{\prime}=\int_{0}^{1} d_{q} x x^{t-1} \frac{\varphi(q x)}{\varphi\left(q^{s} x\right)}
$$

is known to reproduce the integral

$$
\lim _{q \rightarrow 1} \mathcal{I}_{q}^{\prime}=\int_{0}^{1} d x x^{t-1}(1-x)^{s-1} .
$$


It remains to study the factor in front of $\mathcal{I}_{q}^{\prime}$ in (5.7). We first note that $\vartheta_{q}(z)$ is closely related to the Jacobi theta function $\theta_{1}(x, \tau)$ defined as

$$
\theta_{1}(x, \tau)=-e^{\frac{\pi \mathrm{i}}{4} \tau} 2 \sin (\pi x) \prod_{n=1}\left(1-e^{2 \pi \mathrm{i} n \tau} e^{2 \pi \mathrm{i} x}\right)\left(1-e^{2 \pi \mathrm{i} n \tau} e^{-2 \pi \mathrm{i} x}\right)\left(1-e^{2 \pi \mathrm{i} n \tau}\right) .
$$

Indeed, the relation between $\vartheta_{q}(z)$ and $\theta_{1}(x, \tau)$ is

$$
\vartheta_{q}\left(e^{2 \pi \mathrm{i} x}\right)=\mathrm{i} e^{\pi \mathrm{i} x} e^{-\frac{\pi \mathrm{i}}{4} \tau} \theta_{1}(x, \tau), \quad q=e^{2 \pi \mathrm{i} \tau} .
$$

In order to study the limit $q \rightarrow 1^{-}$, or equivalently $\tau \rightarrow 0, \operatorname{Im}(\tau)>0$, we may use the modular transformation property of $\theta_{1}(x, \tau)$,

$$
\theta_{1}(x, \tau)=\mathrm{i}(-\mathrm{i} \tau)^{-\frac{1}{2}} e^{-\frac{\pi \mathrm{i}}{\tau} x^{2}} \theta_{1}(x / \tau,-1 / \tau) .
$$

It follows that

$$
\theta_{1}(s \tau, \tau)=\mathrm{i}(-\mathrm{i} \tau)^{-\frac{1}{2}} e^{-\pi \mathrm{i} s^{2} \tau} \theta_{1}(s,-1 / \tau) \sim-\mathrm{i}(-\mathrm{i} \tau)^{-\frac{1}{2}} e^{-\frac{\pi \mathrm{i}}{4 \tau}} 2 \sin (\pi s),
$$

leading to

$$
\vartheta_{q}\left(q^{s}\right) \sim(-\mathrm{i} \tau)^{-\frac{1}{2}} e^{-\frac{\pi \mathrm{i}}{4 \tau}} 2 \sin (\pi s) .
$$

It remains to study the asymptotics of $(q, q)_{\infty}^{3}$. To this aim we may use the relation between $(q, q)_{\infty}$ and the Dedekind eta-function,

$$
(q, q)_{\infty}=e^{-\frac{\pi \mathrm{i} \tau}{12}} \eta(\tau)
$$

Using the modular transformation property $\eta(\tau)=(-i \tau)^{-\frac{1}{2}} \eta(-1 / \tau)$, one finds

$$
(q, q)_{\infty}=e^{-\frac{\pi \mathrm{i} \tau}{12}}(-i \tau)^{-\frac{1}{2}} \eta(-1 / \tau)=e^{-\frac{\pi \mathrm{i} \tau}{12}}(-i \tau)^{-\frac{1}{2}} e^{-\frac{\pi \mathrm{i}}{12 \tau}} \prod_{n=1}\left(1-e^{-2 \pi \mathrm{i} / \tau}\right)
$$

which implies

$$
(q, q)_{\infty} \sim e^{-\frac{\pi \mathrm{i}}{12 \tau}}(-i \tau)^{-\frac{1}{2}}
$$

Taken together this yields

$$
\frac{2 \pi \mathrm{i}}{1-q} \frac{\vartheta_{q}\left(q^{s}\right)}{(q ; q)_{\infty}^{3}} \sim \frac{2 \pi \mathrm{i}}{(-2 \pi \mathrm{i} \tau)} \frac{(-\mathrm{i} \tau)^{-\frac{1}{2}}}{(-i \tau)^{-\frac{3}{2}}} 2 \sin (\pi s) \sim 2 \mathrm{i} \sin (\pi s) .
$$

\section{Integral representation of the $T_{N}$ partition function}

For completeness, in this section we summarise the relation between the $T_{N}$ topological string partition function and a matrix integral that looks like the free field representation of $A_{N-1}$ Toda three point function. The derivation of the results can be found in the thesis [77]. Here we will not recall the form of $\mathcal{Z}_{T_{N}}^{\text {top }}$, we refer the interested reader to $[30,32,77]$. 
The relation between the partition function and the integral formulation after specialisation of parameters $v^{a} A_{i}^{(a)} / A_{i-1}^{(a)}=t^{s_{a, i}} P_{2}^{(a+i)}$ as depicted in figure 14 is ${ }^{11}$

$$
\mathcal{I}_{N}=\left(2 \pi \mathrm{i} \frac{\varphi(t)}{\varphi(q)}\right)^{\sum_{a=1}^{N-1} s_{a}} \vartheta_{M} \mathcal{M}(t / q)^{\frac{N(N-1)}{2}} \prod_{a=1}^{N-1} \prod_{I=1}^{s_{a}}\left(y_{I}^{(a)}\right)_{\emptyset}^{\zeta_{a}+1} \mathcal{Z}_{T_{N}}^{\mathrm{top}},
$$

where the $\left(y_{I}^{(a)}\right)_{Y_{a, i}}$ are the appropriate generalisations of $(5.10)$ for $T_{N}$ with $a=1, \ldots, N-1$ and $i=1, \ldots, N-a$. The integral $\mathcal{I}_{N}$ is similarly a generalisation of $\mathcal{I}_{2}$, defined in (4.9),

$$
\mathcal{I}_{N}=\int d_{\mathbf{q}}^{\prime} \mathbf{y} \prod_{a=1}^{N-1} \prod_{I=1}^{s_{a}}\left(y_{I}^{(a)}\right)^{\zeta a} \mathcal{I}_{m_{a}}(y) \mathcal{I}_{a, a}(y) \prod_{a=1}^{N-2} \mathcal{I}_{a, a+1}(y),
$$

where $\mathbf{y}=\left\{y_{I}^{(a)}\right\}$ contains the set of all integration variables,

$$
\begin{aligned}
& \mathcal{I}_{m_{a}}(y)=\prod_{I=1}^{s_{a}} \frac{\varphi\left(v^{2} P_{2}^{(a)} / y_{I}^{(a)}\right)}{\varphi\left(P_{2}^{(a+1)} / y_{I}^{(a)}\right)}, \\
& \mathcal{I}_{a, a}(y)=\prod_{J \neq I=1}^{s_{a}} \frac{\varphi\left(y_{I}^{(a)} / y_{J}^{(a)}\right)}{\varphi\left(t y_{I}^{(a)} / y_{J}^{(a)}\right)}, \quad \mathcal{I}_{a, a+1}(y)=\prod_{I=1}^{s_{a}} \prod_{J=1}^{s_{a+1}} \frac{\varphi\left(t y_{J}^{(a+1)} / y_{I}^{(a)}\right)}{\varphi\left(y_{J}^{(a+1)} / y_{I}^{(a)}\right)},
\end{aligned}
$$

and

$$
\zeta_{a}=\beta\left(a_{1}+a_{2}, e_{a}\right)-\beta^{2} s_{a+1}+\beta^{2}\left(s_{a}-1\right), \quad s_{N}=0,
$$

with $s_{a}=\sum_{i=1}^{N-a} N_{a, i}$. The vectors $e_{a}$ are the simple roots of the algebra $\mathfrak{s l}_{N}$, the inner products are taken with respect to the Cartan matrix $\kappa_{a b}=\left(e_{a}, e_{b}\right), a_{2}=\left(a_{2}^{(1)}, \ldots, a_{2}^{(N-1)}\right)$ and $\left(a_{2}, e_{a}\right)=a_{2}^{(a)}-a_{2}^{(a+1)}$. The $(N-1)$ component vectors $a_{i}$ in equation (C.5) are related to the gauge theory parameters $P_{1}, P_{2}, P_{3}$ through

$$
\frac{P_{1}^{(a)}}{P_{1}^{(a+1)}}=\frac{q}{t} q^{\beta\left(a_{1}, e_{a}\right)}, \quad \frac{P_{2}^{(a)}}{P_{2}^{(a+1)}}=\frac{t}{q} q^{-\beta\left(a_{2}, e_{a}\right)}, \quad \frac{P_{3}^{(N-a)}}{P_{3}^{(N-a+1)}}=\frac{q}{t} q^{\beta\left(a_{3}, e_{a}\right)}, \quad t=q^{\beta^{2}},
$$

where

$$
a_{3}=a_{1}+a_{2}+\beta \sum_{k=1}^{N-1} s_{k} e_{k} .
$$

Finally, the term $\vartheta_{M}$ in the prefactor is given by

$$
\begin{aligned}
\vartheta_{M}= & \prod_{a=1}^{N-2} \prod_{j>i=1}^{N-a} \prod_{J=1}^{N_{a, j}}\left(\frac{P_{2}^{(a+i)}}{P_{2}^{(a+j)}} t^{-J}\right)^{-\beta^{2} s_{a, i}} \vartheta_{q}\left(\frac{P_{2}^{(a+i)}}{P_{2}^{(a+j)}} t^{-J}, q^{1+\beta^{2} s_{a, i}}\right) \\
& \prod_{j \geq i=1}^{N-a-1} \prod_{I=1}^{N_{a, i}}\left(\frac{P_{2}^{(a+i)}}{P_{2}^{(a+1+j)}} t^{I-1}\right)^{\beta^{2} N_{a+1, j}} \vartheta_{q}\left(\frac{P_{2}^{(a+i)}}{P_{2}^{(a+1+j)}} t^{I-1}, q^{1-\beta^{2} N_{a+1, j}}\right) .
\end{aligned}
$$

\footnotetext{
${ }^{11}$ The parameters of type $A_{m}^{(n)}$ are associated to faces of the $T_{N}$ web diagram. See [30, 32, 77] for definitions and convensions.
} 
We conclude this section by observing that the $q \rightarrow 1$ limit of the integrand of (C.2), up to prefactors that will be worked out and presented in future work, is proportional to the integrand of Warnaar's $A_{N}$ Selberg integral [80, 81] (see also section 5 of [25] for a nice review).

$$
\begin{aligned}
\mathcal{I}_{A_{N-1}}=\int \prod_{a=1}^{N-1} \prod_{I=1}^{s_{a}} d y_{I}^{(a)}\left(y_{I}^{(a)}\right)^{\beta\left(a_{1}, e_{a}\right)}\left(1-y_{I}^{(a)}\right)^{\beta\left(a_{2}, e_{a}\right)} \\
\prod_{J>I}\left(y_{J}^{(a)}-y_{I}^{(a)}\right)^{\kappa_{a a} \beta^{2}} \prod_{b>a} \prod_{J=1}^{s_{b}}\left(y_{I}^{(a)}-y_{J}^{(b)}\right)^{\kappa_{a b} \beta^{2}},
\end{aligned}
$$

where $\kappa_{a b}$ is the Cartan matrix of the $\mathfrak{s l}_{N}$ algebra defined above.

\section{Summary of important formulae}

We collect here some of the key formulae from our paper. The topological string partition function, as computed in [30], is a function $\mathcal{Z}_{2}^{\text {top }}\left(P_{1}, P_{2}, P_{3} ; t, q\right)$ of three Kähler parameters $P_{i}$, with $i=1,2,3$, as well as the Omega deformation parameters $q$, $t$. In section 2.2 we have shown that the infinite sum inside $\mathcal{Z}_{2}^{\text {top }}$ has a finite radius of convergence and that the result is indeed given by a factorised expression, as was conjectured in [30, 40].

One of our goals has been to clarify the relation to Virasoro conformal blocks expected to emerge in the limit where the deformation parameters $q=e^{-R \epsilon_{1}}, t=e^{R \epsilon_{2}}$ have $R \rightarrow 0$. This limit is nontrivial, as we have shown in section 3 , since the function representing the basic building block of the factorised expression $\mathcal{Z}_{2}^{\text {top }}$ diverges in this limit. In the case $|q|<1,|t|<1$ we have shown in section 5 that the limit

$$
\mathcal{Z}_{2}^{4 \mathrm{~d}}\left(a_{1}, a_{2}, a_{3} ; \beta\right)=\lim _{R \rightarrow 0}(2 \pi i)^{s} \mathcal{M}(t / q)\left(\frac{\varphi(t)}{\varphi(q)}\right)^{s} \mathcal{Z}_{2}^{\text {top }}\left(P_{1}, P_{2}, P_{3} ; t, q\right)
$$

exists if $\beta^{2}=-\epsilon_{2} / \epsilon_{1}$, assuming that the parameters $a_{1}, a_{2}, a_{3}$ and $P_{1}, P_{2}, P_{3}$ are related as

$$
P_{1}^{2}=\frac{q}{t} q^{2 \beta a_{1}}, \quad P_{2}^{2}=\frac{t}{q} q^{-2 \beta a_{2}}, \quad P_{3}^{2}=\frac{q}{t} q^{2 \beta a_{3}}, \quad t=q^{\beta^{2}},
$$

where $a_{3}=a_{1}+a_{2}+s \beta$ with $s \in \mathbb{N}, \varphi(x)$ is the quantum dilogarithm function (A.1) and $\mathcal{M}(x)$ has a definition as a plethystic exponential (A.6). What equation (D.1) implies is that some divergent factors in the asymptotic behaviour of $\mathcal{Z}_{2}^{\text {top }}$ for $R \rightarrow 0$ depend on $s$.

In order to establish the precise relation with Liouville conformal blocks we have used an alternative representation for $\mathcal{Z}_{2}^{\text {top }}$ in section 4 , available in the special cases where

$$
\sqrt{\frac{q}{t}} \frac{P_{2} P_{3}}{P_{1}}=t^{s}, \quad s \in \mathbb{N} .
$$

We have shown for this case that $\mathcal{Z}_{2}^{\text {top }}$ has an alternative representation often called $q$ deformed matrix integral, see also [38, 39], in terms of a function $\mathcal{I}_{2}\left(P_{1}, P_{2}, P_{3} ; t, q\right)$ defined as

$$
\mathcal{I}_{2}=\int \frac{d_{q}^{\prime} y_{1}}{y_{1}} \cdots \frac{d_{q}^{\prime} y_{s}}{y_{s}} \prod_{i=1}^{s} y_{i}^{\zeta+1} \mathcal{I}_{1,1}(y) \mathcal{I}_{m}(y), \quad q^{\zeta+1}=\sqrt{\frac{t}{q}} \frac{P_{1} P_{3}}{P_{2}}
$$


where

$$
\mathcal{I}_{1,1}(y)=\prod_{i \neq j=1}^{s} \frac{\varphi\left(y_{i} / y_{j}\right)}{\varphi\left(t y_{i} / y_{j}\right)}, \quad \mathcal{I}_{m}(y)=\prod_{i=1}^{s} \frac{\varphi\left(P_{2}^{2} v^{2} / y_{i}\right)}{\varphi\left(1 / y_{i}\right)} .
$$

The integrals $\int d_{q}^{\prime} y_{1} \cdots d_{q}^{\prime} y_{s} \prod_{i=1}^{s} y_{i}^{-1}$ are variants of the Jackson integral ${ }^{12}$ defined for meromorphic functions $M(y)$ of $s$ variables $y=\left(y_{1}, \ldots, y_{s}\right)$ as

$$
\int \frac{d_{q}^{\prime} y_{1}}{y_{1}} \cdots \frac{d_{q}^{\prime} y_{s}}{y_{s}} M(y):=(2 \pi \mathrm{i})^{s} \sum_{\substack{R_{1}, \ldots, R_{s} \in \mathbb{N} \\ R_{1}>R_{2}>\ldots R_{s}}} \underset{\substack{\operatorname{Res}_{R} \\ y=y_{R}}}{ } M(y),
$$

where $y_{R}=\left(t^{s-1} q^{R_{1}}, t^{s-2} q^{R_{2}}, \ldots, q^{R_{s}}\right)$. The precise relation between $\mathcal{Z}_{2}^{\text {top }}$ and $\mathcal{I}_{2}$ has been shown to be

$$
\mathcal{Z}_{2}^{\mathrm{top}}\left(P_{1}, P_{2}, P_{3} ; t, q\right)=\frac{t^{-\frac{1}{2} s(s-1)(\zeta+1)}}{(2 \pi i)^{s} \mathcal{M}(t / q)}\left(\frac{\varphi(q)}{\varphi(t)}\right)^{s} \mathcal{I}_{2}\left(P_{1}, P_{2}, P_{3} ; t, q\right)
$$

Representing $\mathcal{Z}_{2}^{\text {top }}$ via equation (D.7) offers another way to study the limit (D.1). Despite the fact that all the ingredients in the definition of $\mathcal{I}_{2}\left(P_{1}, P_{2}, P_{3} ; t, q\right)$ diverge in this limit, we have found that a finite limit exists for this function proportional to the Selberg integral, giving an independent confirmation for (D.1).

The function $\mathcal{Z}_{2}^{4 \mathrm{~d}}\left(a_{1}, a_{2}, a_{3}\right)$ has thereby been found to be

$$
\begin{aligned}
\mathcal{Z}_{2}^{4 \mathrm{~d}}\left(a_{1}, a_{2}, a_{3} ; \beta\right)= & \left(\frac{\beta^{1-\beta^{2}}}{2 \pi \mathrm{i}} \Gamma\left(\beta^{2}\right)\right)^{-s} \frac{\Gamma_{\beta}(\beta)}{\Gamma_{\beta}((s+1) \beta)} \\
& \frac{\Gamma_{\beta}\left(\beta^{-1}+2 a_{1}\right)}{\Gamma_{\beta}\left(\beta^{-1}+2 a_{1}+s \beta\right)} \frac{\Gamma_{\beta}\left(\beta-2 a_{2}\right)}{\Gamma_{\beta}\left(-2 a_{2}+(1-s) \beta\right)} \frac{\Gamma_{\beta}\left(2 \beta^{-1}-\beta+2 a_{3}\right)}{\Gamma_{\beta}\left(2 \beta^{-1}-(s+1) \beta+2 a_{3}\right)},
\end{aligned}
$$

the three point conformal block of the Virasoro algebra with $c=1-6\left(\beta-\beta^{-1}\right)^{2}<1$ central charge. It is interesting to note that a similar limit exists for $|t|>1,|q|<1$ giving Liouville conformal blocks. Relations of the form (D.1) have previously been proposed in [30, 40]. However, the references above have neither identified a renormalisation prescription giving a finite limit such as (D.1), nor correctly identified the precise relation (D.2) between the parameters.

To understand the $T_{2}$ vertex as a building block, we have compared this in section 6 to a much more intensively studied counterpart in the relevant literature, the strip geometry. The main insight gained here has been that in various chambers of the Kähler moduli space, the partition functions agree up to leg factors for the corresponding web diagrams. However, not all of the chambers are covered by the strip diagrams. We have then checked in section 9 that gluing $T_{2}$ vertices gives the correct conformal blocks of higher point functions, explicitly analysing how to glue two such vertices to obtain the partition function of the $5 \mathrm{D}$ theory with $\mathrm{SU}(2)$ gauge group and $N_{f}=4$ fundamental hypermultiplets. We

\footnotetext{
${ }^{12}$ The integration measure appears as such in order to satisfy the identification with the summation (D.6). This has been further discussed in section 4 .
} 
have first reviewed how the partition function of the $\mathrm{SU}(2), N_{f}=4$ theory can be obtained by gluing two strip functions $\mathcal{Z}_{\tau_{1}, \tau_{2}}^{\text {strip }}\left(\mathbf{Q}_{\mathbf{m}} ; t, q\right)$

$$
\mathcal{Z}_{\text {oct }}\left(\mathbf{Q}_{\mathbf{m}}, \mathbf{Q}_{\mathbf{m}^{\prime}} ; t, q\right)=\sum_{\tau_{1}, \tau_{2}}\left(-Q_{B_{1}}^{\prime}\right)^{\left|\tau_{1}\right|}\left(-Q_{B_{2}}^{\prime}\right)^{\left|\tau_{2}\right|} \mathcal{Z}_{\tau_{1}, \tau_{2}}^{\text {strip }}\left(\mathbf{Q}_{\mathbf{m}} ; t, q\right) \mathcal{Z}_{\tau_{2}^{t}, \tau_{1}^{t}}^{\text {strip }}\left(\mathbf{Q}_{\mathbf{m}^{\prime}} ; q, t\right)
$$

The instanton piece in $\mathcal{Z}_{\text {oct }}=\mathcal{Z}_{\text {oct }}^{\text {pert }} \mathcal{Z}_{\text {oct }}^{\text {inst }}$ determined by specialising the Kähler parameters $Q_{B_{1}}^{\prime}=u Q_{B_{1}}^{\prime \prime}$ and $Q_{B_{2}}^{\prime}=u Q_{B_{2}}^{\prime \prime}$ can be recast as a sum over orders in the instanton expansion

$$
\mathcal{Z}_{\text {oct }}^{\text {inst }}\left(\mathbf{Q}_{\mathbf{m}}, \mathbf{Q}_{\mathbf{m}^{\prime}} ; t, q\right)=\sum_{k=0}^{\infty} u^{k} \mathcal{Z}_{k}^{\text {strip }}\left(\mathbf{Q}_{\mathbf{m}}, \mathbf{Q}_{\mathbf{m}^{\prime}} ; t, q\right)
$$

and reproduces the expansion of the corresponding Virasoro conformal block in powers of the cross-ratio.

Gluing $T_{2}$ building blocks requires decorating external legs of the corresponding web diagrams by Young tableaux and we have shown how this yields Virasoro conformal blocks. We have found that this procedure produces a partition function of the form

$$
\mathcal{Z}_{\text {hex }}\left(\mathbf{Q}, \mathbf{Q}^{\prime} ; t, q\right)=\sum_{\lambda_{1}, \lambda_{2}}\left(-Q_{B_{1}}\right)^{\left|\lambda_{1}\right|}\left(-Q_{B_{2}}\right)^{\left|\lambda_{2}\right|} \mathcal{Z}_{\lambda_{1}, \lambda_{2}}^{\mathrm{top}, 2}(\mathbf{Q} ; t, q) \mathcal{Z}_{\lambda_{2}^{t}, \lambda_{1}^{t}}^{\mathrm{top}, 2}\left(\mathbf{Q}^{\prime} ; q, t\right)
$$

depending on the Kähler parameters $\mathbf{Q}, \mathbf{Q}^{\prime}$. By specialising the Kähler parameters $Q_{B_{i}}$ in terms of the instanton expansion parameter $u$, this expression can be recast in a form $\mathcal{Z}_{\text {hex }}=\mathcal{Z}_{\text {hex }}^{\text {pert }} \mathcal{Z}_{\text {hex }}^{\text {inst }}$ where the instanton piece is related to $\mathcal{Z}_{\text {oct }}^{\text {inst }}$ through

$$
\mathcal{Z}_{\text {hex }}^{\text {inst }}\left(\mathbf{Q}, \mathbf{Q}^{\prime} ; t, q\right)=\frac{\mathcal{Z}_{\text {oct }}^{\text {inst }}\left(\mathbf{Q}_{\mathbf{m}}, \mathbf{Q}_{\mathbf{m}^{\prime}} ; t, q\right)}{\mathcal{M}\left(Q_{B_{1}}^{\prime}\right) \mathcal{M}\left(Q_{B_{2}}^{\prime} \frac{t}{q}\right)}
$$

at the first orders in $u$.

Open Access. This article is distributed under the terms of the Creative Commons Attribution License (CC-BY 4.0), which permits any use, distribution and reproduction in any medium, provided the original author(s) and source are credited.

\section{References}

[1] L.F. Alday, D. Gaiotto and Y. Tachikawa, Liouville Correlation Functions from Four-dimensional Gauge Theories, Lett. Math. Phys. 91 (2010) 167 [arXiv:0906.3219] [INSPIRE].

[2] V.A. Alba, V.A. Fateev, A.V. Litvinov and G.M. Tarnopolskiy, On combinatorial expansion of the conformal blocks arising from AGT conjecture, Lett. Math. Phys. 98 (2011) 33 [arXiv: 1012.1312] [INSPIRE].

[3] N. Nekrasov, BPS/CFT correspondence V: BPZ and KZ equations from qq-characters, arXiv:1711.11582 [INSPIRE].

[4] D. Gaiotto, $N=2$ dualities, JHEP 08 (2012) 034 [arXiv:0904.2715] [INSPIRE].

[5] D. Gaiotto, G.W. Moore and A. Neitzke, Wall-crossing, Hitchin Systems, and the WKB Approximation, arXiv:0907.3987 [INSPIRE]. 
[6] Y. Tachikawa, A brief review of the 2d/ 4d correspondences, J. Phys. A 50 (2017) 443012 [arXiv: 1608.02964] [INSPIRE].

[7] V.A. Fateev and A.V. Litvinov, On AGT conjecture, JHEP 02 (2010) 014 [arXiv: 0912.0504] [INSPIRE].

[8] A. Mironov and A. Morozov, Proving AGT relations in the large-c limit, Phys. Lett. B 682 (2009) 118 [arXiv: 0909.3531] [INSPIRE].

[9] L. Hadasz, Z. Jaskolski and P. Suchanek, Proving the AGT relation for $N_{f}=0,1,2$ antifundamentals, JHEP 06 (2010) 046 [arXiv: 1004.1841] [INSPIRE].

[10] A. Mironov, A. Morozov and S. Shakirov, Towards a proof of AGT conjecture by methods of matrix models, Int. J. Mod. Phys. A 27 (2012) 1230001 [arXiv:1011.5629] [InSPIRE].

[11] A. Mironov, A. Morozov and S. Shakirov, A direct proof of AGT conjecture at beta $=1$, JHEP 02 (2011) 067 [arXiv: 1012.3137] [INSPIRE].

[12] V.A. Fateev and A.V. Litvinov, Integrable structure, W-symmetry and AGT relation, JHEP 01 (2012) 051 [arXiv:1109.4042] [INSPIRE].

[13] H. Kanno, K. Maruyoshi, S. Shiba and M. Taki, $W_{3}$ irregular states and isolated $N=2$ superconformal field theories, JHEP 03 (2013) 147 [arXiv:1301.0721] [INSPIRE].

[14] S. Mironov, A. Morozov and Y. Zenkevich, Generalized Jack polynomials and the AGT relations for the SU(3) group, JETP Lett. 99 (2014) 109 [arXiv:1312.5732] [INSPIRE].

[15] G.W. Moore, N. Nekrasov and S. Shatashvili, Integrating over Higgs branches, Commun. Math. Phys. 209 (2000) 97 [hep-th/9712241] [INSPIRE].

[16] N.A. Nekrasov, Seiberg-Witten prepotential from instanton counting, Adv. Theor. Math. Phys. 7 (2003) 831 [hep-th/0206161] [INSPIRE].

[17] L. Hollands, C.A. Keller and J. Song, Towards a $4 d / 2 d$ correspondence for Sicilian quivers, JHEP 10 (2011) 100 [arXiv:1107.0973] [InSPIRE].

[18] S.H. Katz, A. Klemm and C. Vafa, Geometric engineering of quantum field theories, Nucl. Phys. B 497 (1997) 173 [hep-th/9609239] [INSPIRE].

[19] S. Katz, P. Mayr and C. Vafa, Mirror symmetry and exact solution of 4-D N=2 gauge theories: 1., Adv. Theor. Math. Phys. 1 (1998) 53 [hep-th/9706110] [InSPIRE].

[20] M. Aganagic, A. Klemm, M. Mariño and C. Vafa, The topological vertex, Commun. Math. Phys. 254 (2005) 425 [hep-th/0305132] [INSPIRE].

[21] A. Iqbal, C. Kozcaz and C. Vafa, The refined topological vertex, JHEP 10 (2009) 069 [hep-th/0701156] [INSPIRE].

[22] H. Awata and H. Kanno, Refined BPS state counting from Nekrasov's formula and Macdonald functions, Int. J. Mod. Phys. A 24 (2009) 2253 [arXiv:0805.0191] [InSPIRE].

[23] R. Dijkgraaf and C. Vafa, Toda Theories, Matrix Models, Topological Strings, and $N=2$ Gauge Systems, arXiv:0909.2453 [INSPIRE].

[24] M.C.N. Cheng, R. Dijkgraaf and C. Vafa, Non-Perturbative Topological Strings And Conformal Blocks, JHEP 09 (2011) 022 [arXiv:1010.4573] [INSPIRE].

[25] R. Schiappa and N. Wyllard, An A(r) threesome: Matrix models, 2d CFTs and $4 d N=2$ gauge theories, J. Math. Phys. 51 (2010) 082304 [arXiv:0911.5337] [INSPIRE]. 
[26] K. Maruyoshi, $\beta$-Deformed Matrix Models and 2d/4d Correspondence, in New Dualities of Supersymmetric Gauge Theories, J. Teschner, ed., pp. 121-157 (2016), DOI [arXiv: 1412.7124] [INSPIRE].

[27] N.C. Leung and C. Vafa, Branes and toric geometry, Adv. Theor. Math. Phys. 2 (1998) 91 [hep-th/9711013] [INSPIRE].

[28] A. Gorsky, S. Gukov and A. Mironov, SUSY field theories, integrable systems and their stringy/brane origin. 2., Nucl. Phys. B 518 (1998) 689 [hep-th/9710239] [inSPIRE].

[29] F. Benini, S. Benvenuti and Y. Tachikawa, Webs of five-branes and $N=2$ superconformal field theories, JHEP 09 (2009) 052 [arXiv:0906.0359] [INSPIRE].

[30] L. Bao, V. Mitev, E. Pomoni, M. Taki and F. Yagi, Non-Lagrangian Theories from Brane Junctions, JHEP 01 (2014) 175 [arXiv:1310.3841] [INSPIRE].

[31] H. Hayashi, H.-C. Kim and T. Nishinaka, Topological strings and $5 d T_{N}$ partition functions, JHEP 06 (2014) 014 [arXiv: 1310.3854] [INSPIRE].

[32] V. Mitev and E. Pomoni, Toda 3-Point Functions From Topological Strings, JHEP 06 (2015) 049 [arXiv: 1409.6313] [INSPIRE].

[33] M. Isachenkov, V. Mitev and E. Pomoni, Toda 3-Point Functions From Topological Strings II, JHEP 08 (2016) 066 [arXiv: 1412.3395] [INSPIRE].

[34] D. Gaiotto and J. Maldacena, The gravity duals of $N=2$ superconformal field theories, JHEP 10 (2012) 189 [arXiv:0904.4466] [INSPIRE].

[35] A. Iqbal and C. Vafa, BPS Degeneracies and Superconformal Index in Diverse Dimensions, Phys. Rev. D 90 (2014) 105031 [arXiv:1210.3605] [INSPIRE].

[36] H. Hayashi and G. Zoccarato, Exact partition functions of Higgsed $5 d T_{N}$ theories, JHEP 01 (2015) 093 [arXiv: 1409.0571] [INSPIRE].

[37] Y. Tachikawa, A review of the $T_{N}$ theory and its cousins, PTEP 2015 (2015) $11 \mathrm{~B} 102$ [arXiv: 1504.01481] [INSPIRE].

[38] M. Aganagic, N. Haouzi, C. Kozcaz and S. Shakirov, Gauge/Liouville Triality, arXiv:1309.1687 [INSPIRE].

[39] M. Aganagic, N. Haouzi and S. Shakirov, $A_{n}$-Triality, arXiv:1403.3657 [INSPIRE].

[40] C. Kozcaz, S. Pasquetti and N. Wyllard, A \& B model approaches to surface operators and Toda theories, JHEP 08 (2010) 042 [arXiv: 1004.2025] [INSPIRE].

[41] A. Iqbal and A.-K. Kashani-Poor, The vertex on a strip, Adv. Theor. Math. Phys. 10 (2006) 317 [hep-th/0410174] [INSPIRE].

[42] O. Aharony and A. Hanany, Branes, superpotentials and superconformal fixed points, Nucl. Phys. B 504 (1997) 239 [hep-th/9704170] [INSPIRE].

[43] O. Aharony, A. Hanany and B. Kol, Webs of $(p, q)$ five-branes, five-dimensional field theories and grid diagrams, JHEP 01 (1998) 002 [hep-th/9710116] [INSPIRE].

[44] A. Iqbal and A.-K. Kashani-Poor, Instanton counting and Chern-Simons theory, Adv. Theor. Math. Phys. 7 (2003) 457 [hep-th/0212279] [INSPIRE].

[45] A. Iqbal and A.-K. Kashani-Poor, $\mathrm{SU}(N)$ geometries and topological string amplitudes, Adv. Theor. Math. Phys. 10 (2006) 1 [hep-th/0306032] [INSPIRE]. 
[46] M. Taki, Refined Topological Vertex and Instanton Counting, JHEP 03 (2008) 048 [arXiv:0710.1776] [INSPIRE].

[47] H. Awata and H. Kanno, Changing the preferred direction of the refined topological vertex, J. Geom. Phys. 64 (2013) 91 [arXiv:0903.5383] [InSPIRE].

[48] Y. Zenkevich, Refined toric branes, surface operators and factorization of generalized Macdonald polynomials, JHEP 09 (2017) 070 [arXiv: 1612.09570] [INSPIRE].

[49] T. Kimura, H. Mori and Y. Sugimoto, Refined geometric transition and qq-characters, JHEP 01 (2018) 025 [arXiv:1705.03467] [INSPIRE].

[50] P. Sulkowski, Crystal model for the closed topological vertex geometry, JHEP 12 (2006) 030 [hep-th/0606055] [INSPIRE].

[51] I.G. Macdonald, Symmetric Functions and Hall Polynomials, Oxford University Press, (1998).

[52] Y. Konishi and S. Minabe, Flop invariance of the topological vertex, Int. J. Math. 19 (2008) 27 [math/0601352] [INSPIRE].

[53] V. Mitev, E. Pomoni, M. Taki and F. Yagi, Fiber-Base Duality and Global Symmetry Enhancement, JHEP 04 (2015) 052 [arXiv: 1411.2450] [INSPIRE].

[54] I. Coman, E. Pomoni and J. Teschner, From quantum curves to topological string partition functions, arXiv: 1811.01978 [INSPIRE].

[55] B. Feigin and E. Frenkel, Quantum W algebras and elliptic algebras, Commun. Math. Phys. 178 (1996) 653 [q-alg/9508009] [INSPIRE].

[56] E. Frenkel and N. Reshetikhin, Deformations of W-algebras associated to simple Lie algebras, q-alg/9708006.

[57] A.B. Zamolodchikov, Three-point function in the minimal Liouville gravity, Theor. Math. Phys. 142 (2005) 183 [hep-th/0505063] [INSPIRE].

[58] S. Ribault and R. Santachiara, Liouville theory with a central charge less than one, JHEP 08 (2015) 109 [arXiv: 1503.02067] [INSPIRE].

[59] T. Eguchi and H. Kanno, Topological strings and Nekrasov's formulas, JHEP 12 (2003) 006 [hep-th/0310235] [INSPIRE].

[60] R. Dijkgraaf and C. Vafa, On geometry and matrix models, Nucl. Phys. B 644 (2002) 21 [hep-th/0207106] [INSPIRE].

[61] B. Eynard and N. Orantin, Invariants of algebraic curves and topological expansion, Commun. Num. Theor. Phys. 1 (2007) 347 [math-ph/0702045] [INSPIRE].

[62] B. Eynard, A short overview of the "Topological recursion", arXiv:1412.3286 [INSPIRE].

[63] K. Iwaki, T. Koike and Y. Takei, Voros Coefficients for the Hypergeometric Differential Equations and Eynard-Orantin's Topological Recursion - Part I: For the Weber Equation, arXiv:1805.10945.

[64] K. Iwaki, T. Koike and Y. Takei, Voros Coefficients for the Hypergeometric Differential Equations and Eynard-Orantin's Topological Recursion - Part II: For the Confluent Family of Hypergeometric Equations, arXiv:1810.02946.

[65] V.S. Adamchik, Contributions to the Theory of the Barnes Function, math/0308086. 
[66] M. Fukuda, Y. Ohkubo and J. Shiraishi, Generalized Macdonald Functions on Fock Tensor Spaces and Duality Formula for Changing Preferred Direction, arXiv:1903.05905 [INSPIRE].

[67] A. Negut, The q-AGT-W relations via shuffle algebras, Commun. Math. Phys. 358 (2018) 101 [arXiv: 1608.08613] [INSPIRE].

[68] I. Coman, M. Gabella and J. Teschner, Line operators in theories of class $\mathcal{S}$, quantized moduli space of flat connections, and Toda field theory, JHEP 10 (2015) 143 [arXiv: 1505. 05898] [INSPIRE].

[69] I. Coman, E. Pomoni and J. Teschner, Toda conformal blocks, quantum groups, and flat connections, Commun. Math. Phys. 375 (2019) 1117 [arXiv:1712.10225] [INSPIRE].

[70] M. Taki, Seiberg Duality, 5d SCFTs and Nekrasov Partition Functions, arXiv:1401.7200 [INSPIRE].

[71] F. Benini, Y. Tachikawa and B. Wecht, Sicilian gauge theories and $N=1$ dualities, JHEP 01 (2010) 088 [arXiv:0909.1327] [inSPIRE].

[72] E. Gava, K.S. Narain, M. Muteeb and V.I. Giraldo-Rivera, $N=2$ gauge theories on the hemisphere $H S^{4}$, Nucl. Phys. B 920 (2017) 256 [arXiv:1611.04804] [INSPIRE].

[73] M. Dedushenko, Gluing II: Boundary Localization and Gluing Formulas, arXiv:1807.04278 [INSPIRE].

[74] S. Pasquetti, Factorisation of $N=2$ Theories on the Squashed 3-Sphere, JHEP 04 (2012) 120 [arXiv: 1111.6905] [INSPIRE].

[75] C. Beem, T. Dimofte and S. Pasquetti, Holomorphic Blocks in Three Dimensions, JHEP 12 (2014) 177 [arXiv:1211.1986] [INSPIRE].

[76] J. Teschner, Liouville theory revisited, Class. Quant. Grav. 18 (2001) R153 [hep-th/0104158] [INSPIRE].

[77] I. Coman-Lohi, On generalisations of the AGT correspondence for non-Lagrangian theories of class S, Ph.D. Thesis, Hamburg University, Hamburg, Germany (2018), DOI [InSPIRE].

[78] A.N. Kirillov, Dilogarithm identities, Prog. Theor. Phys. Suppl. 118 (1995) 61 [hep-th/9408113] [INSPIRE].

[79] T.H. Koornwinder, Jacobi functions as limit cases of q-ultraspherical polynomials, J. Math. Anal. Appl. 148 (1990) 44.

[80] S.O. Warnaar, A Selberg integral for the Lie algebra $A_{n}$, arXiv:0708.1193.

[81] S.O. Warnaar, The sl $3_{3}$ Selberg integral, Adv. Math. 224 (2010) 499 [arXiv:0901.4176]. 